Nova Southeastern University

\title{
Effect of Lighting on Performance of Tasks Requiring Near Vision in Older Adults
}

\author{
Karen Leigh James \\ Nova Southeastern University
}

This document is a product of extensive research conducted at the Nova Southeastern University College of Health Care Sciences. For more information on research and degree programs at the NSU College of Health Care Sciences, please click here.

Follow this and additional works at: https://nsuworks.nova.edu/hpd_ot_student_dissertations

Part of the Occupational Therapy Commons

All rights reserved. This publication is intended for use solely by faculty, students, and staff of Nova Southeastern University. No part of this publication may be reproduced, distributed, or transmitted in any form or by any means, now known or later developed, including but not limited to photocopying, recording, or other electronic or mechanical methods, without the prior written permission of the author or the publisher.

\section{NSUWorks Citation}

Karen Leigh James. 2016. Effect of Lighting on Performance of Tasks Requiring Near Vision in Older Adults. Doctoral dissertation. Nova Southeastern University. Retrieved from NSUWorks, College of Health Care Sciences - Occupational Therapy Department. (51) https://nsuworks.nova.edu/hpd_ot_student_dissertations/51. 
THE EFFECT OF LIGHTING ON PERFORMANCE OF TASKS REQUIRING NEAR VISION IN OLDER ADULTS

\author{
by
}

Karen Leigh James

Submitted in partial fulfillment of the requirements for the degree of

Doctor of Philosophy in Occupational Therapy

Occupational Therapy Department

College of Health Care Sciences

Nova Southeastern University

Fort Lauderdale, Florida 33328

November 2016 


\section{NOVA SOUTHEASTERN UNIVERSITY \\ HEALTH PROFESSIONS DIVISION \\ COLLEGE OF HEALTH CARE SCIENCES \\ OCCUPATIONAL THERAPY DEPARTMENT \\ FORT LAUDERDALE, FL 33328}

This dissertation, written by Karen Leigh James under direction of her Dissertation Committee, and approved by all of its members, has been presented and accepted in partial fulfillment of requirements for the degree of

DOCTOR OF PHILOSOPHY

DISSERTATION COMMITTEE

Max Ito, Ph.D., OTR/L

Date

Rachelle Dorne, Ed.D., OTR/L, CAPS

Date

JoAnne Wright, Ph.D., OTR/L, CLVT, FAOTA

Date 


\title{
NOVA SOUTHEASTERN UNIVERSITY \\ HEALTH PROFESSIONS DIVISION \\ COLLEGE OF HEALTH CARE SCIENCES \\ FORT LAUDERDALE, FL 33328
}

\begin{abstract}
Certification
We hereby certify that this dissertation, submitted by Karen Leigh James, conforms to acceptable standards and is fully adequate in scope and quality to fulfill the dissertation requirement for the Doctor of Philosophy degree.
\end{abstract}

\section{Approved:}

Rick Davenport, Ph.D., OTR/L

Date

$\mathrm{PhD}$ in Occupational Therapy Program Director

Wendy Stav, Ph.D., OTR/L, FAOTA

Date

Managing Director of Fort Lauderdale Occupational Therapy Programs

Peter Taylor, Ph.D.

Date

Associate Dean of Academic Affairs

Stanley Wilson, PT, Ed.D., CEAS

Date

Dean, College of Health Care Sciences 
Copyright Page 


\begin{abstract}
With age, more light is needed in order to function, but the effect of lighting on occupational performance has received little attention in occupational therapy. The purpose of the study was to determine if lighting affects older adults' ability to perform selected occupational tasks, which require near vision and if lighting levels affects their perceived effort while performing selected occupational tasks. A quasi-experimental, repeated measures design was used with a convenience sample recruited from a retirement community. Thirty participants met the inclusion criteria, which included visual and cognitive screening. The mean age of the 30 participants was 83 years, and most $(80 \%)$ were female. Participants completed three occupational tasks (reading a prescription label, sorting pills, and sorting dark colored socks) under three different lighting levels (low, $M=103$; medium, $M=127$; and high, $M=397$ footcandles [fc]) presented in random order. Participants were placed in three groups based on order of lighting presentation and completed each set of tasks four times: a trial session, followed by three timed sessions. Participants were asked to rate their perceived effort under each lighting level using a self-report scale. Descriptive statistics were used to examine completion times, groups, and lighting levels. In general, participants required longer to complete occupational tasks and reported more effort when utilizing medium light levels $(M=127 \mathrm{fc})$ provided by ambient lighting and a floor lamp and performed best under high lighting levels $(M=397 \mathrm{fc})$ provided by ambient lighting as well as a floor lamp and task lamp. The results suggest lighting may have affected performance and perceived effort of older adults while completing the visually challenging tasks.
\end{abstract}




\section{Acknowledgements}

I am grateful for many who have helped me throughout my dissertation journey. I would like to thank my dissertation committee, Dr. Max Ito, Chair, and Dr. Rachelle Dorne and Dr. JoAnne Wright for their continuous encouragement and support. I am appreciative of the individual contributions of each as I completed this study. Dr. Ito provided with me with a "just right" challenge, allowing me to work independently yet providing advice as needed. Dr. Dorne challenged me to remain occupation focused in the midst of very technical information, and Dr. Wright continually encouraged me and spurred me on in my pursuit of this topic. I would also like to thank Dr. Rob Kennedy for his assistance with statistical analysis. I am grateful for the time he invested in answering my questions and his dedication to seeing this project through to completion. I appreciate the director of the retirement community who allowed me to complete the study within the community, and I am grateful to the residents of the community for their willingness to participate in the study. Without their participation, this study would not have been possible.

I am overwhelmingly thankful for the continuous support of my family. They believed in me, encouraged me, and assisted me in so many ways. Their love and support carried me through the past seven years, and I am eternally grateful for them. 


\section{Table of Contents}

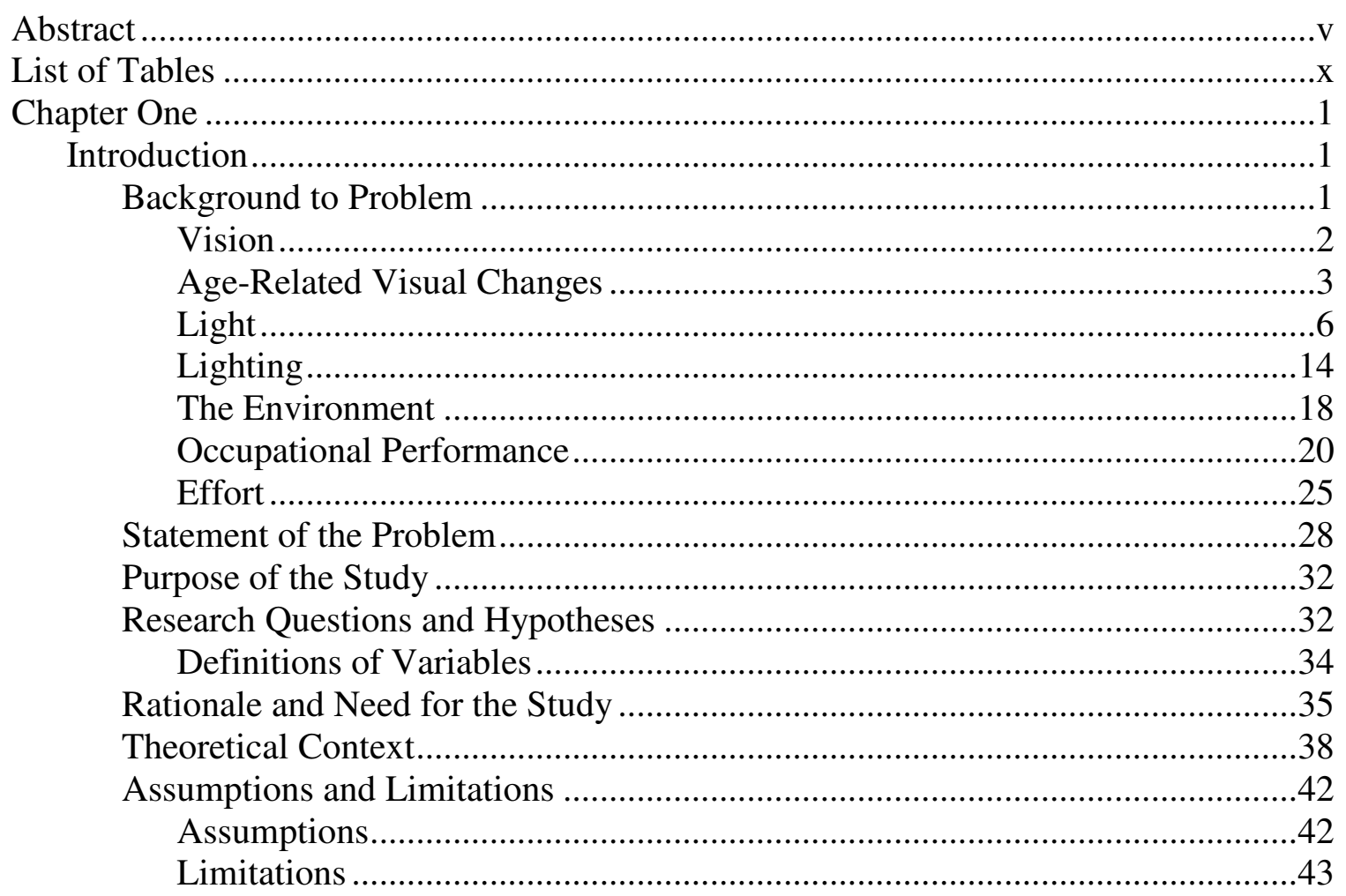

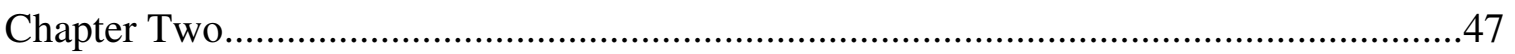

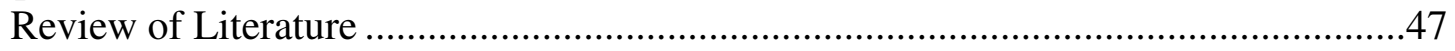

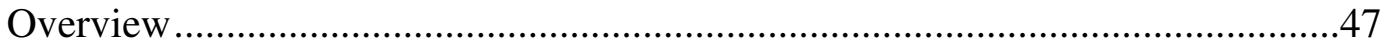

The Effects of Lighting on Individuals with Low Vision..................................47

The Effects of Lighting on Individuals with Normal Vision ...............................54

Studies of Lighting in the Home ...................................................................58

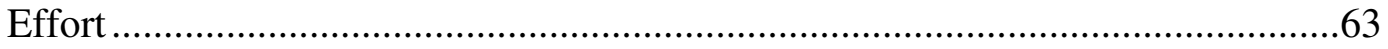

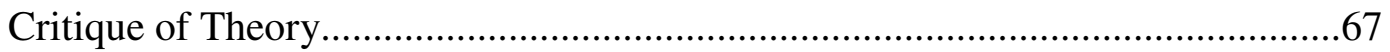

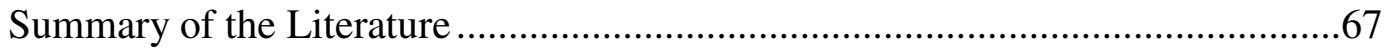

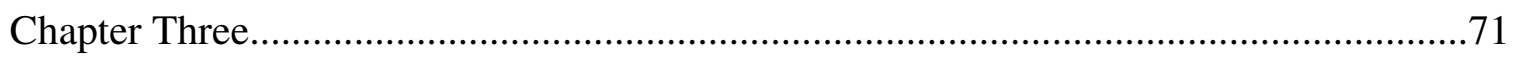

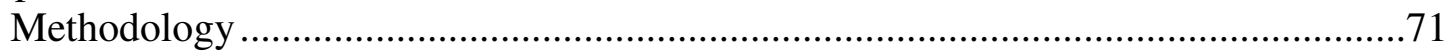

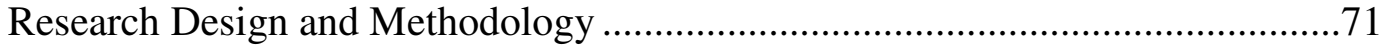

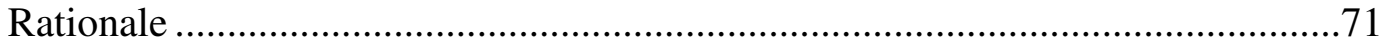

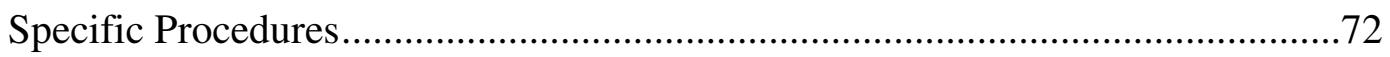

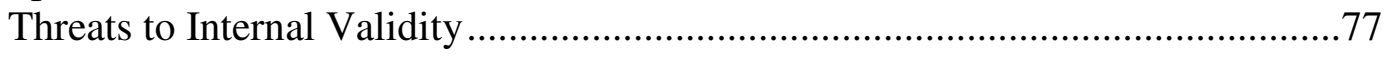

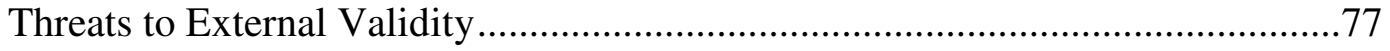

Strengths and Weaknesses of the Design...................................................... 78

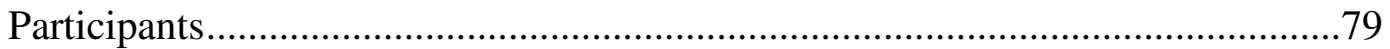

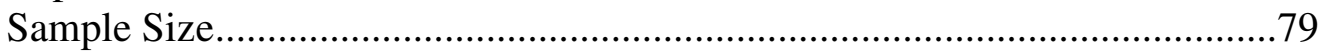

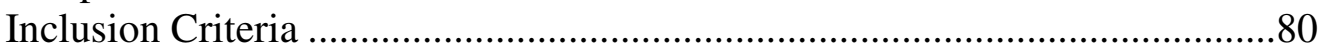

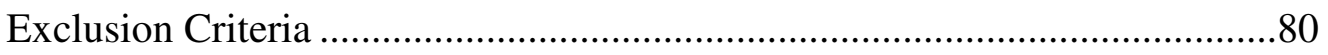

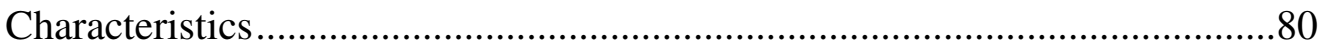




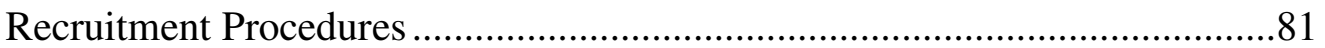

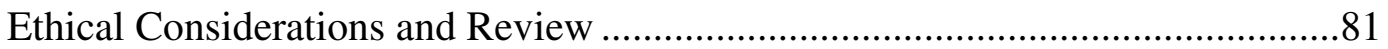

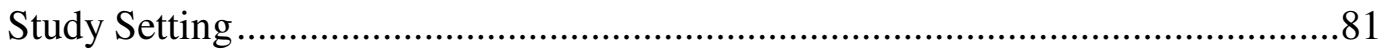

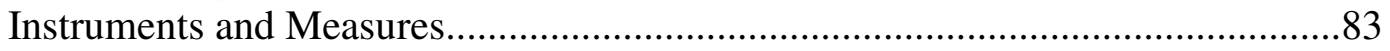

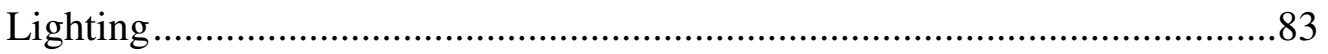

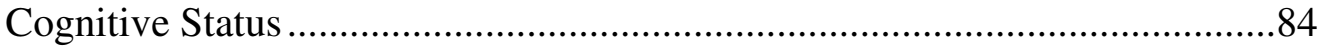

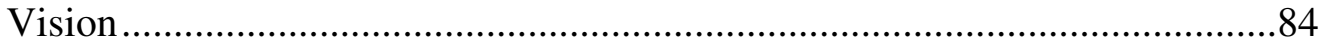

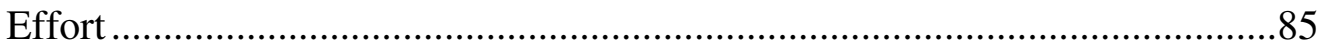

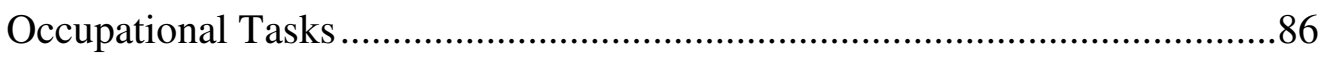

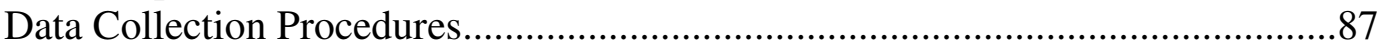

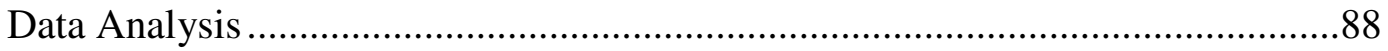

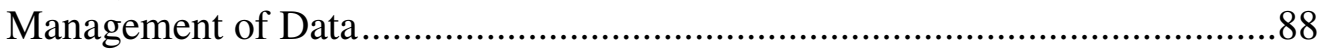

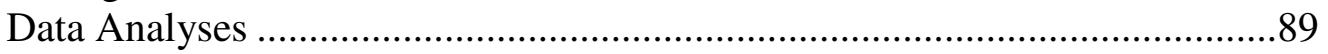

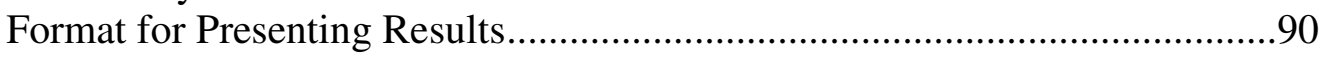

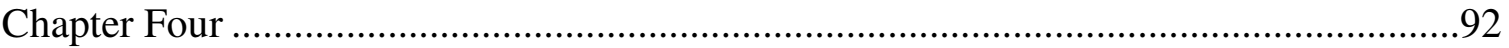

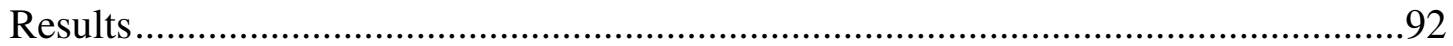

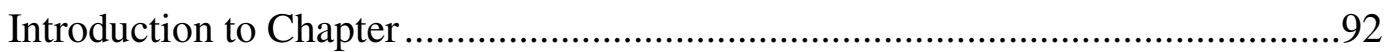

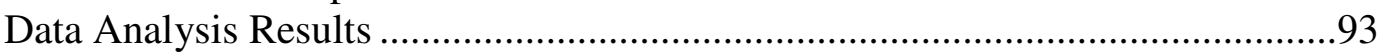

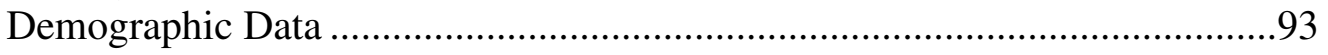

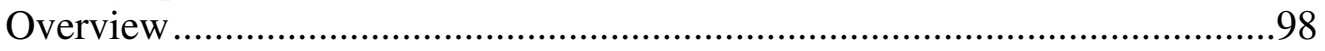

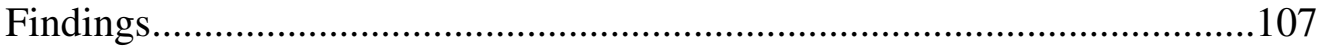

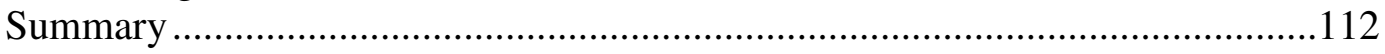

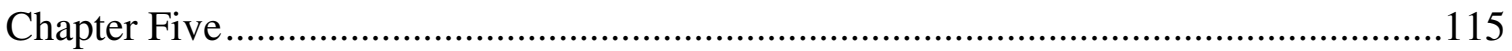

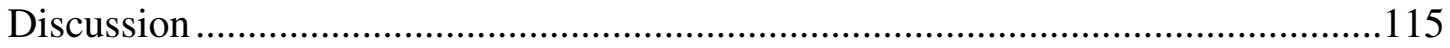

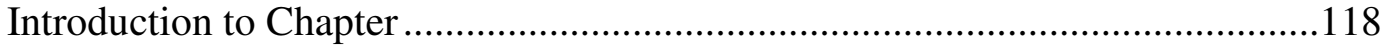

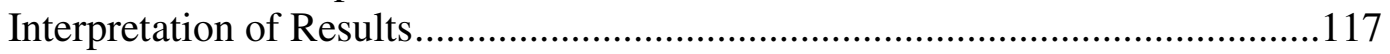

The Variables ............................................................................................ 118

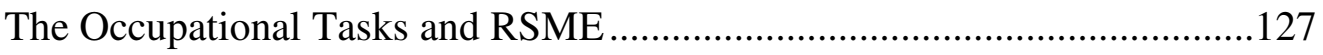

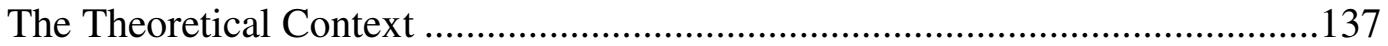

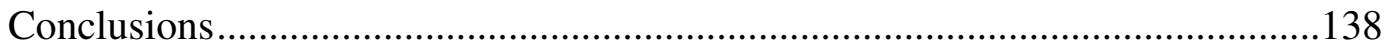

Implications for Occupational Therapy Practice ..........................................140

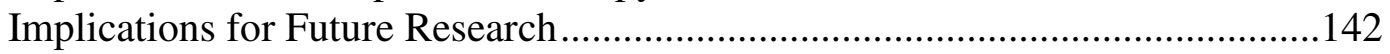

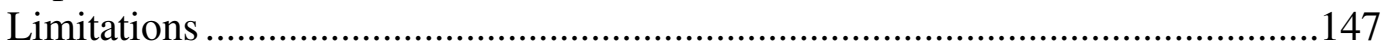

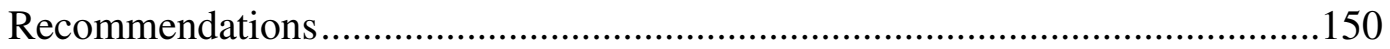

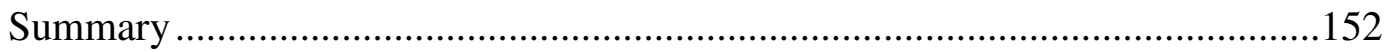

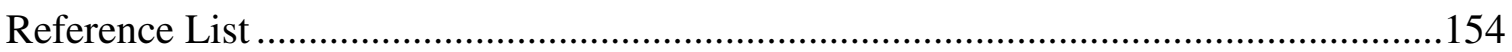

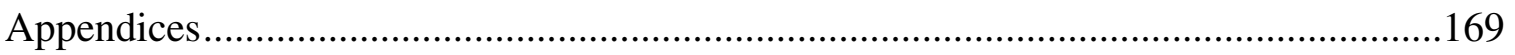

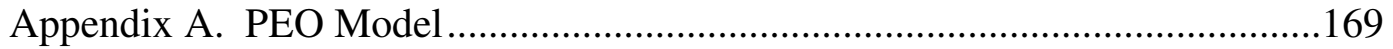

Appendix B. Flyer for Informational Meeting...............................................170

Appendix C. Flyer for Research Study ........................................................171

Appendix D. Demographic Information Sheet ..........................................172

Appendix E. Study Record ................................................................. 175

Appendix F. Cards for Order of the Lighting Conditions................................176

Appendix G. Contents of Testing Sets..........................................................177 


\section{List of Tables}

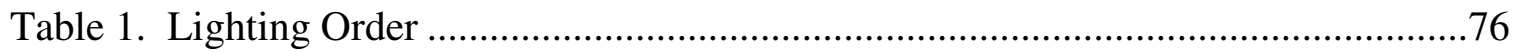

Table 2. MMSE-2 BV and Colengrander Mixed Contrast Card Group Results .............94

Table 3. General Demographic Data ................................................................95

Table 4. Eye Disease ...................................................................................................96

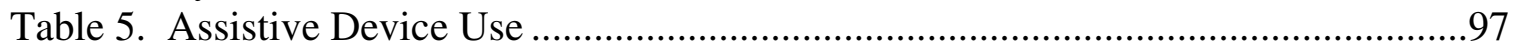

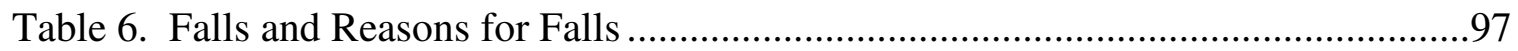

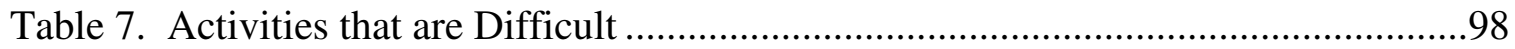

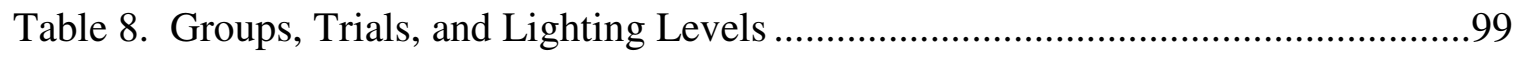

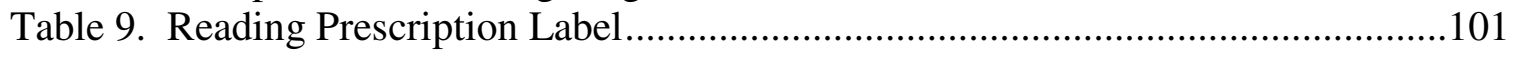

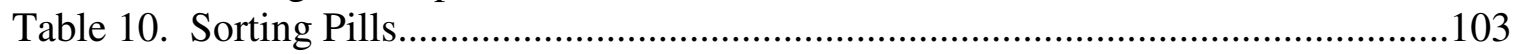

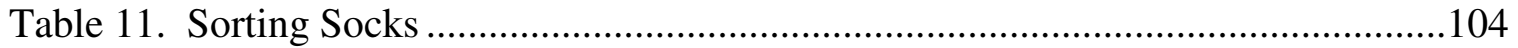

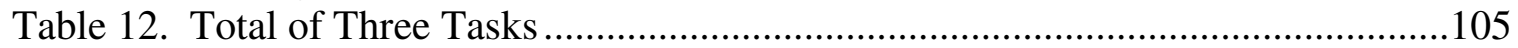

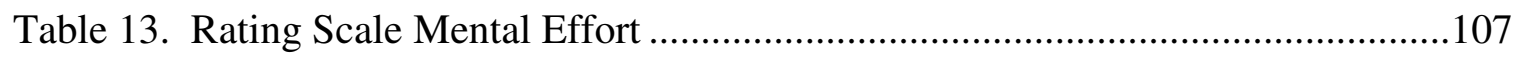

Table 14. Overall Lighting Means Requiring Greatest and Least Time........................107

Table 15. Lighting Levels Requiring Greatest and Least Time and Effort ...................114

Table 16. Recommended Groups, Trials, and Lighting Levels ...................................148 


\section{Chapter 1: Introduction}

\section{Background to the Problem}

The population of the United States (US) is aging, and the population of older Americans is expected to grow (Administration on Aging, 2014) because those reaching 65 years of age have an average life expectancy of an additional 19.3 years. In 2013, the number of adults 65 years of age or older in the US, the most recent figures available, was greater than 44.7 million, and by 2060 , this number is expected to more than double to 98 million. Due in large part to the aging of the baby boomers, this increase in the aging population will have significant ramifications for the US.

As people age, changes in bodily function occur that limit their ability to participate in daily activities (Administration on Aging, 2014). One of these changes is declining vision (Noell-Waggoner, 2004), which affected 7\% of Americans 65 years of age and older in 2013 (Administration on Aging, 2014). As a result of these changes, older adults need more light to compensate (Boyce, 2003; Kolanowski, 1992; Krusen, 2010; Moller, 2008; Noell-Waggoner, 2004; Perlmutter, 2012; Rizzo, 2007; Young, 2012). As the population ages, so does the prevalence of those with age-related visual changes and the challenges that accompany those changes. Consequently, we need to move beyond research that compares the performance of young adults versus older adults for tasks performed in a research laboratory. Instead, research that focuses on the practical needs of older adults and the difficulties they encounter when performing everyday tasks in everyday environments is needed.

Further, society would benefit from research that contributes to a theoretical framework through which age-related visual changes and the impact of age-related visual 
changes on every day function can be studied (Owsley, 2011). This chapter will discuss vision and age-related visual changes, light and lighting, lighting standards and the aging eye, the effects of the environment on the aging population, occupational performance, and effort as it relates to occupational performance in older adults.

\section{Vision}

The ability to see is set in motion when light reflects off objects in the environment and is received into the visual system in which both optical and neural processes are responsible for vision (Sekular \& Sekular, 2000). When reflected light reaches the eye, it moves through the cornea (the fixed lens) before entering through the pupil, an opening in the iris. The muscular nature of the iris allows the pupil to constrict and dilate, thereby controlling the passage of light. As light goes through the pupil, it is focused by the variable lens (hereafter referred to as the lens) onto the retina. Muscles attached to the lens contract and relax, changing its shape, and enabling the eye to focus on near and distant objects. Light entering the eye is converted into electrical impulses by the retina, and these impulses are sent to the brain via the optic nerve. The retina contains light sensitive cells, rods, and cones (Marieb, 2001; National Framework for Energy Efficiency, 2009; Nolan, 2002). Cone cells, which provide color vision, are most highly concentrated in the center of the retina (macula) and its center point (the fovea). More abundant in number than cone cells are rod cells (Mogk, 2011), which provide only black and white vision.

Cone cells are ineffective at the lowest light levels, and vision is taken over by the very sensitive rod cells. For this reason, the eye only detects black and white in dark conditions. Rod vision is also known as scotopic vision. Conversely, at very high light 
levels, saturated rod cells can no longer contribute to vision; therefore, cone cells assume responsibility for vision. Cone vision is referred to as photopic vision. At medium levels of light, both rods and cones are operational and contribute to vision. Vision that occurs as a result of the activation of both rod and cone cells is termed mesopic vision (Fosse \& Valberg, 2004; National Framework for Energy Efficiency, 2009).

\section{Age-Related Visual Changes}

As a person ages, optical and neural changes in the eye result in a significant decline in nearly every aspect of visual function (Rizzo, 2007; Salvi, Akhtar, \& Currie, 2006). Common changes in vision include reduced accommodation (presbyopia), reduced visual acuity, reduced contrast sensitivity, increased time for adaptation to darkness, decline in color discrimination due to decreased sensitivity of cone cells and yellowing of the lens, and reduced visual field (Sekular \& Sekular, 2000). In order to understand the impact of age-related changes on visual function, one must understand the anatomical changes that occur in the eye, including changes in the cornea, the lens, the pupil, and the retina (Nolan, 2002; Oklahoma Geriatric Education Center, 2009).

As a part of the natural aging process, increasingly less light reaches the retina because the iris weakens and the pupil becomes smaller (called senile miosis; Sekular \& Sekular, 2000). Consequently, the retina of a 60 year old receives only one third of the light that arrives on the retina of a 20 year old (Christenson, 1990). The transparent, flexible lens is held in place by zonular fibers, and to focus on objects, the lens must change shape. As the ciliary muscle around the lens contracts, the lens thickens, allowing the eye to focus on objects (accommodation). When an object moves away, the ciliary muscle relaxes, the lens becomes thinner, and the focusing system relaxes. However, 
with age, the ciliary muscles atrophy and the lens hardens, resulting in decreased accommodation (presbyopia), which prevents one from focusing on objects at close range or adapting to sharp changes in brightness. The cornea thickens, and consequently, light is scattered as it enters the eye, which reduces the contrast of the retinal image (Oklahoma Geriatric Education Center, 2009; Scheiman, 2011). Also contributing to the scattering of light is the yellowing of the lens, which diminishes color discrimination, rendering older adults less sensitive to colors on the blue end of the color spectrum (Smith, 2015).

In addition to optical changes within the eye, aging causes neural changes that also contribute to changes in vision. Neural changes include a reduction in the number of neurons in the retina. The retina is made of layers of neurons, including a layer of photoreceptors that convert light energy into a neural signal. As the number of retinal cells diminishes, the retina thins, and the remaining cells often become irregular in shape. Normally, photoreceptors in the retina are angled in such a way that light entering the eye hits the top of the photoreceptors, limiting the response to light scatter. As the eye ages, the photoreceptors become disarranged and abnormally oriented, negatively affecting their ability to respond to light entering the eye. As a result, the photoreceptors in the aging eye must not only respond to light as it normally enters the eye but also must respond to light that bounces off of retinal structures and light that scatters as it enters the eye. This stray light also lands on the top of the photoreceptors, increasing the amount of light they must manage and contributing to glare in the aging eye (Nolan, 2002). The fovea is the center of the retina where the retinal image is cast when the eye focuses on an object, and it is responsible for acuity (detection of fine details). The fovea contains the 
cones, the color sensitive photoreceptor cells. With age, the fovea loses cells, resulting in decreased color discrimination (Salvi et al., 2006). Particularly difficult to differentiate are dark colors (black, brown, and navy) and pastels (Noell-Waggoner, 2004). The eye is also less able to discriminate colors (hues) in the blue-green range, especially under diminished lighting (Sekular \& Sekular, 2000) because blue light (short wavelength) is filtered away by the aging eye (Moller, 2008), and the yellowing of the lens alters the quality of light entering the pupil. Consequently, dark shades of navy, brown, and black are distinguishable only under more intense light (Christenson, 1990).

Poor color discrimination also contributes to poor contrast sensitivity. Contrast sensitivity is the measure of the smallest amount of contrast a person can detect between an object in the foreground and the background. As color discrimination diminishes with age, so does contrast sensitivity, making it difficult for one to detect a light gray object against a white background for example.

A combination of anatomical and neural changes in the aging eye contributes to decreased acuity or the ability to resolve detail. Cell loss in the fovea and increased light scatter and glare within the eye decrease acuity as does decreased transparency in the vitreous at the back of the eye. Contrast sensitivity also plays a role in visual acuity as a stimulus with higher contrast is more visible than one with low contrast (Nolan, 2002). However, many older adults with normal visual acuity have difficulty performing daily living tasks due to decreased contrast in their environment, and lighting levels can affect contrast sensitivity (Rizzo, 2007). In good lighting conditions, a 75 year old requires twice as much contrast on a task as a young person, and adults over the age of 90 
required six times as much contrast (Brabyn, Haegerstrom-Portnoy, Schneck, \& Lott, 2000).

Many of the visual changes that occur as part of the natural aging process also occur in the same continuum as disease processes. For example, changes in the lens occur as part of the natural aging process but also arise as a cataract develops, and increased protein deposits that are present as a person ages may or may not be of the type (drusen) that is associated with age-related macular degeneration (Owsley, 2011). It is important to understand and distinguish normal, age-related changes from those that are disease related. This dissertation study involved participants with age-related visual changes. However, the literature review includes studies involving those with aging vision as well as those with low vision due to eye disease because both populations are often included in studies. Studies involving participants with aging vision are limited, so information gained from studies of those with vision loss contributed to this dissertation study.

\section{Light}

Light is a vital, natural phenomenon that allows us to see the world (National Framework for Energy Efficiency, 2009). The quality, quantity, and intensity of light affect how we view and enjoy our surroundings (Ruya, 2015). Good lighting is comforting and provides a sense of safety and security (American Lighting Association, 2012). However, age-related changes in the eye result in less light reaching the retina, which is detrimental to vision, making it difficult for older adults to function with less lighting. Consequently, to accommodate for age-related changes in vision, more light is required (Christenson, 1990; Figueiro, 2001; Noell-Waggoner, 2004; Rizzo, 2007). 
While light is provided naturally through sources, such as the sun, the advent of electrical sources of light has rendered us less dependent on natural light (National Framework for Energy Efficiency, 2009). As a result, an understanding of electrical light and light sources requires an explanation of terminology associated with light and lighting and is pertinent to this dissertation study.

Lighting terminology. In order to fully realize the implications of lighting on task or occupational performance an understanding of lighting terminology is important. Terms that are pertinent to the dissertation study are provided below along with their definitions. A discussion of light and lighting follows the list.

1. Ambient lighting. Ambient lighting is "lighting throughout the area that produces general illumination" (Illuminating Engineering Society of North America [IESNA], 1998, p. 47).

2. Color rendering. Color rendering is "general expression for the effect of a light source on the color appearance of objects in conscious or subconscious comparison with their color appearance under a reference light source (IESNA, 1998, p. 48).

3. Color rendering index (CRI). CRI is "a measure of a lamp's ability to render colors accurately" (Pegasus Lighting, 2016, p. 11).

4. Compact fluorescent. Compact florescent is "a generic name /used for a whole family of small, single-ended fluorescent lamps with a folded, bridged, or spiral glass tube design and with high color rendering $(\mathrm{CRI}>80)$ and a long life (>8000 hours)" (Pegasus Lighting, 2016, p. 12). 
5. Correlated color temperature (CCT). Correlated color temperature is "a measure of the color appearance or hue of a light source which helps describe the apparent 'warmth' (reddish) or 'coolness' (bluish) of that light source" (Pegasus Lighting, 2016, p. 12).

6. Disability glare. Disability glare is "the effect of stray light in the eye whereby visibility and visual performance are reduced" (IESNA, 1998, p. 47).

7. Discomfort glare. Discomfort glare is "glare producing discomfort. It does not necessarily interfere with visual performance or visibility" (IESNA, 1998, p. 47).

8. Efficacy. Efficacy is "lumens per watt" (American Lighting Association, 2012, p. 14).

9. Footcandles. Footcandles are "the amount of light reaching a subject" (American Lighting Association, 2012, p. 14).

10. Fluorescent lamp. A fluorescent lamp is "a low-pressure mercury electricdischarge lamp (light bulb) in which a phosphor coating on the inside of the glass tubing transforms some of the ultraviolet energy created inside the lamp into visible light" (Pegasus Lighting, 2016, p. 20).

11. Glare. Glare is "luminance within the visual field that is sufficiently greater than the luminance to which the eyes are adapted that causes annoyance, discomfort, or loss in visual performance and visibility" (IESNA, 1998, p. 49).

12. Halogen lamp. Halogen lamp is "a type of incandescent lamp (light bulb) that contains halogen gases (such as iodine, chlorine, bromine and fluorine), which slow the evaporation of the tungsten filament" (Pegasus, 2016, p. 23). 
13. Illuminance. Illuminance is "a measure of the luminous flux reaching a surface given in terms of lumen $/ \mathrm{m}^{2}$ (= lux)" (Fosse \& Valberg, 2004, p. 26).

14. Kelvin temperature scale. The Kelvin temperature scale is "a temperature scale that references to absolute zero ( 0 degrees Kelvin), which, in theory, is the absence of all thermal energy" (Pegasus Lighting, 2016, p. 31).

15. Kelvin. Kelvin is "a unit of measurement used to describe the hue of a specific light source” (Tire Rack, n.d., p. 1).

16. Light emitting diode (LED). Light emitting diode is "a small electronic device that lights up when electricity is passed through it" (Pegasus, 2016, p. $34)$.

17. Lighting. Lighting is "the application of light" (Rizzo, 2007, p. 2).

18. Light. Light is the "electromagnetic radiation of any wavelength that travels in a vacuum with a speed of about 186,281 miles (300,000 kilometers per second)" (Light, n.d.).

19. Light level. Light level is "the amount of light measured in a plane" (Illumenate, n.d., p. 1).

20. Light meter. A light meter is "a small and often portable device for measuring illumination" (Light meter, n.d.).

21. Lumens. Lumens are "the amount of light that a light source produces" (American Lighting Association, 2012, p. 14).

22. Luminance. Luminance is "a measure of the intensity of the light emitted or reflected from a surface" (Fosse \& Valberg, 2004, p. 391). 
23. Luminous flux. Luminous flux is "the overall light output (luminous radiant power) of a lamp or luminaire measured in lumens" (Pegasus, 2016, p. 38).

24. Lux. Lux is "the International System (SI) unit of illuminance. It is the illumination on a surface one square meter in area on which there is a uniformly distributed flux of one lumen, or the illumination produced at a surface all points of which are at a distance of one meter from a uniform point source of one candela" (IESNA, 1998, p. 50).

25. Mesopic vision. Mesopic vision is "a combination of photopic vision and scotopic vision in low lighting, which functions due to a combination of rod and cone cells in the eye" (Innovative Lighting, Inc., 2013, p. 1).

26. Photopic vision. Photopic vision is "vision under well-lit conditions, which provides for color perception, and which functions primarily due to cone cells in the eye" (Innovative Lighting, Inc., 2013, p. 1).

27. Scotopic vision. Scotopic vision is "monochromatic vision in very low light, which functions primarily due to rod cells in the eye" (Innovative Lighting, Inc., 2013, p. 1).

28. Spectral power distribution. Spectral power distribution is "a pictorial representation of the radiant power emitted by a light source at each wavelength or band of wavelengths in the visible region of the electromagnetic spectrum (360 to 770 nanometers)" (IESNA, n.d., p. 1).

29. Task lighting. Task lighting is "lighting which is directed at a specific surface or area for providing illumination for specific visual tasks" (IESNA, 1998, p. 51). 
30. Tungsten. Tungsten is "a chemical element found in nature as a steel-gray metal that has the highest melting point of all the non-alloyed metals is, therefore, often used to make the filament in incandescent light bulbs" (Pegasus Lighting, 2016, p. 58).

31. Wattage. Wattage is "the amount of electricity consumed by a light source" (American Lighting Association, 2012, p.14).

Description of light. Four terms are used to describe light, and comprehension of these terms is important to an understanding of studies of light and lighting: luminous flux, luminous intensity, illuminance (illumination), and luminance. Luminous flux is the amount of energy emitted in all directions per second from a light source (lamp), and its unit of measure is the lumen (Architectural Acoustics and Lighting, n.d.; National Framework for Energy Efficiency, 2009). Luminous intensity is the measure of how much luminous flux (lumens) is emitted from a lamp in a specific direction, and its unit of measure is the candela (Architectural Acoustics and Lighting, n.d.; National Framework for Energy Efficiency, 2009).

The intensity of light measured on a plane at a specific location is illuminance. The international unit (SI) of illuminance is lux or lumen per square meter (National Framework for Energy Efficiency, 2009), and the English measure is the footcandle or lumen per square foot. Footcandles falling on a horizontal surface are referred to as horizontal footcandles, whereas the footcandles falling on a vertical surface are termed vertical footcandles (Energy Trust of Oregon, 2013). One footcandle is the equivalent of 10.76 lux; however, for convenience, when converting, it is usually rounded to 10 (Rensselaer Polytechnic Institute, 2013). 
Illumination is the process that occurs when light hits a solid surface. The human eye cannot see illumination; however, it does see the brightness of light or luminance (candela/m2), the measure of the amount of light emitted from a surface in a particular direction (Environmental Protection Agency, 1995; National Framework for Energy Efficiency, 2009). While luminance is a measure of the intensity of light reflected from a surface (measured in candela/square meter), illuminance is the light imposed on a surface (Fosse \& Valberg, 2004).

The inverse square law for light intensity states that the intensity of light observed from a light source falls off as the square of the distance from the object. Essentially, this law implies that when the distance between a bulb and the object being viewed is decreased, the intensity of the light increases. For example, if the distance between a bulb and the object being viewed is cut in half, the intensity of the bulb increases by four, meaning a 75 watt bulb becomes 300 watts. This law has important implications for the placement of light in the environment as it suggests that one could use floor lamps and/or task lamps that can be placed closer to the task rather than increasing the wattage of the bulb (Scheiman, Scheiman, \& Whittaker, 2007).

Qualities of light. Important qualities of light to consider in relation to vision are glare, uniformity of illuminance, color rendition, and color temperature (NoellWaggoner, 2004). When luminance in the visual field is too bright, it results in glare (Environmental Protection Agency, 1995). Glare can come directly from a light source, or it can occur when light is reflected off a surface (Noell-Waggoner, 2004). One experiences discomfort glare when the intensity of light prevents the eye from naturally adapting, resulting in discomfort and reducing the ability to see. Disabling glare occurs 
when light is so intense it blocks vision, significantly impairing vision and preventing one from discerning detail. An individual who feels the need to squint on a bright but cloudy day is experiencing discomfort glare, whereas an individual rendered helpless when faced with oncoming headlights is experiencing disabling glare (Young, 2012).

The uniformity of illuminance refers to how evenly light is distributed in a space. The placement and condition of fixtures can affect the uniformity of illuminance as an uneven distribution of light can impact visual performance. Moreover, color rendition refers to the ability of a light source to accurately reflect the true color of an object. The color rendering index, a scale of 0 to 100 , compares the effect of light on the perceived color appearance of an object. The higher the CRI, the more true color appears, whereas the lower the CRI, the more distorted color appears (Environmental Protection Agency, 1995; Krusen, 2010).

Color temperature of a light source is the "color of a blackbody radiator at a given absolute temperature, expressed in Kelvins" (Environmental Protection Agency, 1995, p. 4). It is the measurement of "warmth" or "coolness" provided by the light source with lower numbers representing warm light (more yellow/orange) and higher numbers representing cool light (more blue). Light sources many consider warm, such as incandescent bulbs, are approximately 2700 Kelvin. Many prefer warmer colors, but cooler colors may also be beneficial, especially in distinguishing dark colors, such as black, navy, brown, and green (Noell-Waggoner, 2004; Washington State Department of Ecology, n.d.). 


\section{Lighting}

The application of light, referred to as lighting (Rizzo, 2007), is provided through a luminaire (fixture), consisting of a lamp (bulb) and parts to hold the lamp, distribute the light and connect the lamp to a power source (Environmental Protection Agency, 1995). Ambient lighting, also known as general lighting, refers to the overall lighting within a space. Often provided by ceiling or wall-mounted fixtures, it provides a comfortable level of brightness that allows one to safely move about a space. Task lighting illuminates a specific area where work, such as reading or balancing a checkbook, is being performed. It can be provided by track lighting, under-cabinet lighting, or portable lamps. Accent lighting is light added to draw visual attention to a selected area, such as a painting, and it is provided by track, recessed, or wall-mounted fixtures (American Lighting Association, 2012; Young, 2012).

Lighting is provided by various types of bulbs (lamps). Incandescent bulbs are general service bulbs that are inexpensive and obtainable in a variety of wattages and shapes. Available in clear or frosted finish, these bulbs are utilized in most fixtures, emitting a yellow-white light in all directions (American Lighting Association, 2012). Incandescent bulbs are the most common and the least expensive bulbs, but they do not provide as much light or lumens per watt (LPW) as other bulbs (Krusen, 2010). About $10 \%$ of the energy consumed by incandescent bulbs results in visible light; the other $90 \%$ is radiated as heat (Rizzo, 2007). Incandescent light is good for task lighting as it is easily directed, but when used in ambient lighting, it can pool, resulting in shadows and glare (Watson, 2001). 
Halogen bulbs are incandescent lamps in which a tungsten filament is sealed in a quartz capsule containing a small amount of halogen gas. Halogen bulbs last significantly longer than standard incandescent bulbs (Washington State Department of Energy, n.d.). They produce a brighter and whiter light and provide more light per watt than incandescent bulbs (American Lighting Association, 2012).

Light emitting diode bulbs, available in a variety of designs, are presently one of the most energy efficient bulbs, five times more energy efficient and lasting up to 25 times longer than incandescent bulbs. They can be used in place of incandescent, tungsten, and halogen bulbs, and while they are presently more expensive, they are more cost efficient, so they may become the predominant bulb utilized in residential homes in the future.

Fluorescent light bulbs are more energy efficient and last 10 to 20 times the length of incandescent bulbs (American Lighting Association, 2012). Fluorescent bulbs produce light by passing an electric current through a glass tube with a tiny amount of mercury gas. The phosphor coat on the inside of the glass converts energy to visible light. A ballast is needed to start the lamp and ensure the precise flow of electric current (Washington State Department of Energy, n.d.). While older lamps used magnetic ballasts and a single type phosphor, newer lamps use electronic ballasts and a tri-phospor. The tri-phosphor renders better color, and the electronic ballast eliminates the flicker and hum of the fluorescent bulb (Figueiro, 2001; Rizzo, 2007; Washington State Department of Ecology, n.d.), which has been linked with headaches, eye strain, and other health conditions (Veitch \& McColl, 2001). 
Fluorescent bulbs provide a high level of diffused light (Moller, 2008), are inexpensive, and energy efficient (Watson, 2001). Fluorescent lighting is a good choice in lighting because it provides uniformity and brings levels closest to the intensity of daylight (Halloin, 1995). Further, fluorescent bulbs have warm color tones and excellent color rendering properties (Washington State Department of Energy, n.d.), and they produce less heat than incandescent bulbs. While they are more expensive to buy, they are less expensive to operate (Noell-Waggoner, 2004). Widely used in commercial buildings for many years, T8 fluorescent bulbs with electronic ballasts are becoming increasingly common in residential settings as they turn on instantly, are very efficient, and do not hum (American Lighting Association, 2012).

Small fluorescent bulbs, referred to as compact fluorescents (CFL), were designed to replace incandescent bulbs and are being used progressively more in many types of light fixtures. They last six to 10 times longer and use $75 \%$ less energy than standard incandescent bulbs. Additionally, they are available in dimmable bulbs and are shatterproof (American Lighting Association, 2012; Krusen, 2010; Washington State Department of Energy, n.d.).

Efficacy and efficiency. Efficacy refers to the efficiency with which a lamp converts electrical energy into light and is expressed in lumens per watt. Lamps with higher efficacy require less energy to light a space (Environmental Protection Agency, 1995; National Framework for Energy Efficiency, 2009). Wattage measures electrical consumption of the bulb; it does not measure light output of the bulb (American Lighting Association, 2012). In the United States, light bulbs have historically been purchased and utilized based on wattage, which was displayed on the package. However, in 2011, the 
Federal Trade Commission mandated new packaging for light bulbs, and in an effort to allow consumers the opportunity to purchase the most efficient bulb to meet their needs, the new packages indicate the number of lumens (brightness) provided by the bulb. Each package must contain a "lighting facts" label, which provides the following information about the bulb: brightness, energy cost, life expectancy of the bulb, light appearance (color temperature), wattage, and if the bulb contains mercury (Federal Trade Commission, 2010, 2011).

Additionally, the Energy Independence and Security Act of 2007 established new efficiency standards that were phased in beginning in 2012. The Act does not ban traditional incandescent bulbs but requires that they utilize at least $25 \%$ less energy (watts). Available in a wide range of choices in color and brightness, the newer bulbs are also designed to last longer than traditional bulbs. The traditional 100 watt incandescent and 75 watt incandescent bulbs were largely phased out in 2012 and 2013, respectively; similarly, the traditional 40 watt and 60 watt incandescent bulbs were phased out in January 2014 (United States Department of Energy, 2012).

Lighting standards and the aging eye. Lighting enhances visual performance by rendering objects and tasks more visible. Lighting affects visibility by increasing the contrast sensitivity of the visual system enabling low contrasts between objects or tasks and the background to be more easily seen (IESNA, 1998). The IESNA recommends a minimum of 5 horizontal footcandles for general ambient lighting, and a minimum of 20 fc for casual reading or general work at a desk or countertop. Moreover, for more serious reading or study, a minimum of $50 \mathrm{fc}$ is advised (Conservation Technology of Illinois, 2014). However, for adults over the age of 65, IESNA recommends $100 \mathrm{fc}$ for 
reading/studying and $150 \mathrm{fc}$ for kitchen counter activities (IESNA, 2010). Nonetheless, lighting is not one size fits all, but rather, lighting needs are unique and individualized (American Lighting Association, 2011; Halloin, 1995; Perlmutter, 2012; Young, 2012). Adults with aging eyes frequently complain of difficulty seeing in poor lighting conditions (Owsley, 2011). As age-related changes occur, presbyopia (decreased accommodation) is the only condition that can be treated by corrective lenses. All other conditions must be managed through environmental modifications, such as changes to lighting (Noell-Waggoner, 2004). As stated, IESNA recommends higher light levels for those 65 years of age and older (IESNA, 2010). As the eye ages, rather than being transmitted, more light is absorbed by the lens, and is consequently not available for vision. Thereby, the lens of an 80 year old absorbs 10 times more light than a 20 year old (Sekular \& Sekular, 2000). However, Noell-Waggoner (2004) proposed that lighting requirements for the elderly are not well understood, and her statement is validated in that experts and researchers disagree on the degree of increased lighting needed. For example, IESNA recommends twice as much lighting for older adults (IESNA, 2010), but others recommend up to 10 times as much light for older adults (Christenson, 1990; Kolanowski, 1992; Moller, 2008; Noell-Waggoner, 2004; Watson, 2001; Young, 2012).

\section{The Environment}

The environment provides the context for occupational performance (Cooper, Letts, Rigby, Stewart, \& Strong, 2005) and often influences function or performance, especially for older adults (Rizzo, 2007). People continually interact with their environment, and the person-environment interaction can either enhance or interfere with one's overall well-being. Environmental barriers often diminish the person-environment 
fit. However, environmental modifications are often more easily achieved than changes to a person (Cooper et al., 2005) and can potentially improve functional outcomes (Law, 2002) and safety (Paul \& Liu, 2012).

Lighting is an environmental feature with important implications for safety and function (Barbur \& Konstantakopoulou, 2012; Barstow, Bennett, \& Vogtle, 2011; Chu, Kaldenberg, \& Huefner, 2009; Kuyk \& Elliott, 1999; Paul \& Liu, 2012; Reed, Lowrey, \& Vallis, 2006; Stevens, 2005; Stevens, Holman, \& Bennett, 2001; Tideiskaar, 2003; Van Bemmel, Vandenbroucke, Westendorp, \& Gussekloo, 2005; Woodland \& Hobson, 2003; Wyman et al. 2007). Visual performance worsens at lower ambient lighting levels and can be encumbering for older adults (Barbur \& Konstantakopoulou, 2012). Poor lighting has been identified as a hazard in many homes (Erkal, 2010; Nazarko, 2008; Stevens et al., 2001; van Bemmel et al., 2005), and it can interfere with the ability to safely navigate through the environment and increases the risk of falls (Brown \& Jacobs, 2011; "6 Low Cost," 2015; Tideiskaar, 2003; Woodland \& Hobson, 2003). Approximately $30 \%$ of older adults fall each year (Mackintosh, Fryer, \& Sutherland, 2007; Paul \& Liu, 2012), and as a result, they often experience changes in lifestyle, such as injury, a decrease in activity level, and quality of life (Centers for Disease Control and Prevention, 2013; Paul \& Liu. 2012). Further, poor lighting has been found to decrease the rate of ambulation and increase difficulty in navigating objects in the environment in those with and without visual impairment (Kuyk \& Elliott, 1999; Reed et al., 2006) while adequate lighting can improve one's ability to identify hazards in the environment and perform daily activities (Chu et al., 2009). 
Lighting has the ability to improve one's living environment visually, aesthetically, and functionally (Rizzo, 2007). As people age, they are more dependent on the environment to compensate for changes in their vision, and proper lighting is a key element in helping them compensate for age-related visual changes (IESNA, 1998). However, to be effective, lighting needs to be directed, adjusted, and flexible (American Lighting Association, 2011; Halloin, 1995; Perlmutter, 2012; Young, 2012), and for older adults, light levels need to be consistent and even; light needs to be directed at the task, not the eyes (Noell-Waggoner, 2004). Because older adults often spend so much time indoors (Horgas, Wilms, \& Balts, 1998; Kolanowski, 1992), lighting is an important aspect of the home environment, yet it has not received much consideration by occupational therapists (Gendeman, Perlmutter, \& Baum, 2010; Perlmutter et al., 2013) until recently when Perlmutter (2013) developed a home lighting assessment.

\section{Occupational Performance}

According to the Occupational Therapy Practice Framework (OTPF), Third Edition, occupations are the purposeful, meaningful, daily activities in which people engage (American Occupational Therapy Association [AOTA], 2014). They are situation specific (Christiansen \& Baum, 2005), have a perceived utility to the person engaging in them, and may involve the completion of multiple activities. Occupational performance is the act of doing and accomplishing an occupation that results from the transactional experience among a person, the context and the activity (AOTA, 2014). Occupational performance is comprised of an objective component, which is observable behavior associated with the occupation as well as the subjective component, which is the meaning and feeling associated with the occupation. To measure occupational performance one 
must consider both objective and subjective components (McColl \& Pollock, 2005). A task is defined as work related to the development of occupational performance skills or a group of activities related to the accomplishment of a specific goal (Jacobs \& Simon, 2015). In the dissertation study, participants were asked to complete three functional, goal-directed activities. While the activities were objective, they did not necessarily hold a subjective meaning to the participants. Therefore, the investigator chose to use the term occupational tasks in reference to the activities participants were asked to perform.

While the ability to engage in meaningful occupations is important to the health and well-being of older adults (Berger \& Kaldenberg, 2008), normal aging changes may adversely affect occupational performance and quality of life (Pizzi \& Smith, 2010). Hayase et al. (2004) studied activities of daily living (ADL) and instrumental activities of daily living (IADL) performance in 4398 individuals between the ages of 3 and 93 years of age and found ADL and IADL performance began to gradually decline after the age of 50. Moreover, among 65 to 74 year olds, $3 \%$ needed assistance with ADL and $6 \%$ required assistance with $\mathrm{IADL}$, whereas after the age of $75,11 \%$ required assistance with ADL and 20\% required assistance with IADL as indicated by the 2012 National Health Interview Survey (Adams, Kirzinger, \& Martinez, 2013). Further, the United States Department of Health and Human Services (2014) reported limitations and the need for help with self-care, mobility, and purposeful household activities increases with age. Among 65 to 69 year olds, $11 \%$ require help with self-care and mobility, and $12 \%$ report limitations with household activities while $41 \%$ of 85 to 89 year olds require help with self-care and mobility, and 54\% report limitations with household activities. 
Limitations in IADL often precede ADL limitations (Barberger-Gateau, Rainville, Letenneur, \& Dartigues, 2000) and may prompt the need for assistance or affect one's ability to live independently (Orellano, Colon, \& Arbesman, 2012). One factor that limits IADL performance is impairments in vision (Berger \& Porell, 2008; Grue et al., 2009; Ishihara, Ishihara, Nagamachi, Osaki, \& Hiramatsu, 2004; Sloan, Ostermann, Brown, \& Lee, 2005). As one ages, the quality of vision diminishes due to normal, agerelated changes and age-related visual disorders. Because the prevalence of visual changes increase with age, the issue has serious implications for older adults striving to maintain their independence and age in place (Perlmutter, 2013).

Because visual function is an important part of IADL performance (Berger \& Porell, 2008; Grue et al., 2009; Ishihara et al., 2004; Sloan et al., 2005), this dissertation study will address three occupational tasks that require visual abilities and with which older adults experience difficulty: selecting clothing by color (Barstow et al., 2011; Brunnstrom, Sorensen, Alsterstad, \& Sjostrand, 2004; Cook, 2009; Owsley, McGwin, Sloane, Stalvey, \& Wells, 2001), reading a prescription label and sorting medications (Henriques, Costa, \& Cabrita, 2012; Lam, Elliott, \& George, 2011; MacLaughlin et al., 2005; Owsley et al., 2001; Skomrock \& Richardson, 2010; Windham et al., 2005; Yasein, Barghouti, Irshaid, \& Suleiman, 2013).

In a qualitative study, Barstow et al. (2011) examined occupational performance in 22 adults 50 years of age and older with varying levels of vision loss (non-correctable visual acuity of 20/30 to 20/100) for the purpose of describing difficulties with occupational performance and home safety perspectives. The semi-structured interview questions specifically addressed illumination and contrast as these are factors that 
influence visibility in the environment. Five categories of home safety were identified as imposing difficulty, and lighting was one of the categories. All participants described lighting as key to occupational performance and home safety, and they recognized the importance of optimal lighting for occupational performance. The need for extra light was mentioned, and several participants reported using flashlights to achieve extra light when performing tasks. Some participants explained that they ceased performing activities that require good near vision or coordination of vision and movement of body parts due to feeling unsafe. Other participants reported difficulty with distinguishing colors of clothing and shoes, mentioning the need for increased lighting in storage areas to help with matching clothing and shoes. Barstow et al. (2011) concluded that lighting needs to be more closely addressed and in greater detail during home safety assessments, particularly in individuals with varying degrees of vision loss.

Older adults consume $30 \%$ of the 1.1 billion prescriptions written each year (Hayes, 2005). Consequently, non-adherence to and poor management of medication routines are identified problems that present major health concerns (Henriques et al., 2012; Lam et al., 2011). These problems may be due in part to aging vision (Yasein et al., 2013), which makes it difficult for older adults to read prescription labels, differentiate tablet colors, and open medication vials (MacLaughlin et al., 2005).

Windham et al. (2005) assessed the relationship between vision and the ability to implement a medication routine. Three hundred thirty-five healthy women, aged 73 to 82 years, were given a bottle of aspirin and a prescription bottle with a mock antibiotic and asked to fill a clear pill box with four compartments labeled, "morning," "lunch," "dinner," and "bed" at their usual pace, allowing up to 4 minutes. Only 56\% $(n=187)$ 
placed both pills correctly, yet only $2 \%$ reported difficulty with the task. However, none of those who placed the pills incorrectly were among the $2 \%$ who reported difficulty with the task. Windham et al. (2005) concluded that because roughly half of the high functioning, community dwelling older women in their study had difficulty with medication management, the number of older adults in the general population who experience difficulty with medication routines is likely much higher. Consequently, they proposed this is a problem that needs further investigation.

In a randomized control trial, Skomrock and Richardson (2010) utilized 60 young adults, 18 to 26 years of age, to investigate how age-related changes in color vision affected older adults' ability to take medication. Thirty participants served as the control group while 30 participants in the experimental group wore yellowed-lens glasses to simulate age-related visual changes. Both groups of participants were given a prescription bottle with 80 colored beads (10 each of red, orange, yellow, green, blue, purple, white, and brown) and were asked to sort them by color. No time limit was imposed during the test. Although lighting was not assessed, the study was conducted in a room without windows, utilizing a 60 watt desk lamp for light. The number sorted correctly and the colors that were mistaken were recorded for each participant, and upon completion of the task, participants were asked to rate the difficulty of the task on a scale from 1 (easy) to 5 (difficult). They were also asked which colors were most difficult to distinguish. The control group was $100 \%$ accurate with sorting all colors while the experimental group was $100 \%$ correct with only the colors red and orange. Those in the experimental group had the most difficulty distinguishing purple (average correct $25.7 \%$ ) and brown (average correct 31.2\%). Aside from red and orange, the experimental group 
scored highest with identification of yellow (average correct $80 \%$ ) and white (average correct $83.8 \%$ ). When asked to rate difficulty, the control group rated difficulty at an average score of 1.04 while the experimental group's average difficulty score was 4.02 . Skomrock and Richardson (2010) found their results were consistent with previous studies in which older adults had the most difficulty distinguishing colors in the blueviolet range as well as differentiating white from gray-toned colors (December Communications, n.d.), affirming that age-related changes in color vision negatively affect compliance with medication routines.

The investigator's selection of occupational tasks for this dissertation study was validated by the aforementioned studies. The difficulty older adults experience when selecting clothing has been documented (Barstow et al., 2011; Brunnstrom et al., 2004; Cook, 2009; Owsley et al., 2001) as have the difficulties with reading a prescription label and sorting medications (Henriques et al., 2012; Lam et al., 2011; MacLaughlin et al., 2005; Owsley et al., 2001; Skomrock \& Richardson, 2010; Windham et al., 2005; Yasein et al., 2013). The impact that lighting has on the older adults' ability to complete these occupational tasks requiring near vision or the difficulty they experience in completing these tasks under different lighting levels has not been studied in the literature.

\section{Effort}

Effort is the energy utilized to do something ("Effort," n.d.). Yeo and Neal (2004) describe effort as an invisible, internal, and hypothetical construct that cannot be directly observed; is difficult to measure; and, consequently, is not often studied. Effort intensity describes how hard a person tries when carrying out a task or how much time is spent on a task. Yeo and Neal (2008) proposed effort may be physical or cognitive, and 
they proposed cognitive effort is a limited resource that affects the speed with which individuals process information.

Closely related to effort is efficiency (Zijlstra, 1993). Efficiency ("Efficiency," n.d.) compares the end product with the cost incurred in producing a product, including time spent and energy expended. Through his studies on work efficiency, Zijlstra (1993) stated efficiency corresponds to the amount of effort an individual invests in a task in order to achieve a desired output. Further, he described two different approaches to the concept of mental effort. One approach relates mental effort and attention, espousing the more attention demanding a task is, the more mental effort is required. The second approach describes effort as a process that regulates an individual's psycho-physiological state, keeping it in a state that optimizes task performance and prevents performance from dropping below a certain level. He proposed that while a task imposes a certain workload on an individual, the workload does not automatically prompt expenditure of effort. Rather, expenditure of effort occurs when an individual makes a decision to invest effort in order to execute a task. The amount of effort invested in the task is determined by the individual and is dependent on the complexity of the task and the psycho-physiological state of the individual (Zijlstra, 1993). When a difference exists between an individual's psychophysiological state and the requirement for task completion, then an individual must exert additional effort in order to maintain the required demands for completion of the task. Similarly, Kukla (1972) suggested that the effort an individual invests relates to the perceived difficulty of the task. When a task is perceived as easy, individuals do not put forth much effort to complete it, but as task difficulty increases, more mental effort is mobilized in order to complete the task at a certain performance level. However, when a 
task is deemed impossible to complete, individuals disengage and do not invest effort in completing it (Capa, Audiffren, \& Ragot, 2008).

The aforementioned views on effort, efficiency, and task difficulty as they pertain to functional performance in older adults are supported in the literature. Many older Americans report difficulty with performing ADL and IADL, and often these problems are investigated using subjective measures, such as questionnaires, interviews, or reporting scales that ask individuals to describe or use a scale to rate the difficulty experienced when performing functional tasks (Cohen-Mansfield et al., 2013; Dudgeon et al., 2008; Gitlin, Winter, Dennis, Corcoran, \& Hauck, 2006; Hayase et al., 2004; Langlois et al., 1996; Lin, Beck, Finch, Hummer, \& Master, 2012; Porter, 2007; Rogers, Meyer, Walker, \& Fisk, 1998; Thoren-Jonsson \& Grimby, 2001). While some older adults respond to difficulties by compensating or seeking help, others decrease the frequency with which they perform the tasks, settle for lower performance, or avoid the tasks altogether (Dudgeon et al., 2008; Rogers et al., 1998).

Esposito, Gendolla, and Van der Linden (2014) asked 63 healthy older adults to rate their perceived difficulty and effort investment after performing a memory task. They found a high correlation $(r=0.53, p<.001)$ between subjective task difficulty and the amount of mobilized effort, implying that increased difficulty resulted in increased effort. Further, they found that subjective task demand (perceived difficulty and estimated effort) functioned as a mediator between self-efficacy beliefs and apathy, suggesting that older adults with low self-efficacy may perceive a task as too challenging. Esposito et al. (2014) suggested exerting excessive effort may lead to increased fatigue 
that in turn leads to feelings of apathy. Consequently, when feeling over-challenged by a task, older adults may disengage from the task rather than investing too much effort.

Seeking to understand how well participants understood difficulty in relation to performance, Porter (2007) asked 25 women, 80 to 96 years of age, who lived alone, to describe the difficulty experienced when performing certain basic ADL and IADL. The initial question asked was "Do you have difficulty with (task)?" After allowing the women to respond, the researcher further probed by asking them if extra effort was required and if so, how much extra effort was required and if extra time were needed and if so, why. One of the themes that emerged from the study was "Thinking that difficulty is not the best word for it." Some participants who denied having "difficulty" were asked to further explain and replied that tasks took extra time, extra effort, or both. Another theme that emerged was "Wondering what difficulty really is." In response to being asked to rate difficulty on a numeric scale, several women responded with questions regarding the meaning of the word difficulty, and some chose to redefine the word, describing a task as "harder" or describing their performance as "slower" when completing a task rather than admitting it was difficult. Porter (2007) concluded difficulty is a "subjective perception" that needs exploration, including determining the relationship between extra time, extra effort and difficulty.

\section{Statement of the Problem}

As people age, their visual system changes, affecting their ability to perform IADLs (Berger \& Porell, 2008; Grue et al., 2009; Ishihara et al., 2004; Sloan, Ostermann, Brown, \& Lee, 2005) and prompting the need for more light in order to function (NoellWaggoner, 2004). Of the age-related changes in vision, many of which are in the same 
continuum as eye diseases (Owsley, 2011), only presbyopia can be treated with corrective lenses. Other age-related changes, such as diminished pupil size, yellowing of the lens, and scattering of light as it enters the eye, must be addressed through environmental changes such as adequate lighting (Noell-Waggoner, 2004). Older adults are dependent on the environment to help compensate for their difficulties and inabilities (IESNA, 1998), but at the same time, the environment can place demands that influence performance of IADLs (Barberger-Gateau et al., 2000).

Furthermore, lighting is a particularly important aspect of the physical environment for older adults because of the amount of time they spend indoors (Horgas et al., 1998; Kolanowski, 1992). Proper lighting plays a critical role in improving the living environment and promoting functional independence in older adults (IESNA, 1998; Rizzo, 2007). While adequate lighting helps compensate for visual decline, poor lighting can further compromise an older adult's vision (Davis \& Garza, 2002). IESNA recommends specific lighting levels for varying tasks within the home (Conservation Technology of Illinois, 2014) and increased lighting levels for adults over the age of 65 (IESNA, 2010), but lighting has not received much consideration by occupational therapists (Gendeman et al., 2010) nor has the relationship between lighting, vision, and perceived effort during occupational tasks.

The effect of lighting on performance of individuals with and without visual impairment is well documented by other disciplines (Bowers, Meek, \& Stewart, 2001; Davis \& Garza, 2002; Eldred, 1992; Fosse \& Valberg, 2004; Haymes \& Lee, 2006; Izso, Lang, Laufer, Suplicz, \& Horvath, 2009; Petrofsky \& Cuneo, 2008). However, most studies have been conducted utilizing numeric and reading tests (Bowers et al., 2001; 
Eldred, 1992; Eperjesi, Maiz-Fernandez, \& Bartlett, 2006; Fosse \& Valberg, 2004;

Haymes \& Lee, 2006; Izso, Lang, et al., 2009; Izso, Laufer, \& Suplicz, 2009) rather than functional tasks people complete on a daily basis. Further, as these studies have been conducted by other disciplines, such as architecture, engineering, gerontology, home economics, and psychology, the perspective of an occupational therapist concerned about the broader ADL and IADL challenges of the older adult population is lacking in the literature.

Studies of home environments have identified concerns with lighting (Cullinan, Silver, Gould, \& Irvine, 1979; Cumming et al., 1999; Gill, Williams, Robison, \& Tinetti, 1999; Greene, Sample, \& Fruhauf, 2009; Iwarsson \& Isacsson, 1996) as light levels are often below recommended standards (Charness \& Dijkstra, 1999; Lindner, Rinnert, \& Behrens-Baumann, 2001; Simpson \& Tarrant, 1983). Yet, older adults, lacking awareness of the need for improved lighting, often rely only on ceiling lighting (Lindner et al., 2001), or they use lamps that are not functioning at full capacity due to dustcovered bulbs, dark lamp shades, or non-working fixtures. Furthermore, they rate their lighting as adequate even when it is not (Bakker, Iofel, \& Lachs, 2004).

Because the size of the older adult population continues to grow (Administration on Aging, 2014) and older adults spend much of their time performing occupations indoors (Horgas et al., 1998; Kolanowski, 1992) where lighting is often inadequate (Charness \& Dijkstra, 1999; Lindner et al., 2001; Simpson \& Tarrant, 1983), lighting is an important topic that needs to be addressed (Davis \& Garza, 2002). Nevertheless, lighting, particularly the relationship between lighting and occupational performance, is an area that has not received a great deal of attention from occupational therapists 
(Gendeman et al., 2010). A study documenting observed performance of selected occupational tasks under different lighting levels to ascertain the impact of lighting levels on performance is needed.

Also of importance is the amount of effort older adults perceive is exerted as they perform the occupational tasks under each lighting level. The fact that a person must be willing to exert effort indicates effort is under cognitive control (Zijlstra, 1993). With increasing difficulty, individuals invest more effort in a task (Capa et al., 2008), but when faced with a task that seems too daunting, individuals do not try to perform it (Kukla, 1972). Borell, Lilja, Sviden, and Sadlo (2001) found that, at times, older adults with functional limitations withdrew from performing occupations they deemed too difficult or were unable to perform independently: a sign of reduced hope, which is significant because difficulty with functional activities threatens the quality of life of older adults as well as their ability to live independently and may signify the need to relocate (Gitlin et al., 2006). Consequently, this dissertation study focused on the effect of lighting on older adults' performance of everyday occupational tasks requiring near vision, assessing the amount of time required and the amount of perceived effort expended to complete occupational tasks under the different lighting levels. Knowledge gained from this dissertation study will help further develop guidelines and recommendations for lighting in the homes of older adults, an area that needs to be addressed in order to maximize occupational performance (Krusen, 2010). Chu et al. (2009) hypothesized that optimal lighting will allow older adults to complete occupational tasks more efficiently and with less effort, which in turn will allow them time and energy to participate in other occupational endeavors. Further, the presence of optimal lighting has the potential to 
enhance safety and performance by improving older adults' ability to identify hazards and perform daily activities in the home (Chu et al., 2009). Functional performance declines with age (United States Department of Health and Human Services, 2014) and is often limited by environmental barriers (Gitlin, Mann, Tomits, \& Marcus, 2001). Because lighting has been shown to influence performance (Davis \& Garza, 2002; Izso, Lang, et al., 2009; Izso, Laufer, et al., 2009; Petrofsky \& Cuneo, 2008), it is believed that improving lighting in the home environment has the potential to allow older adults to remain in their homes for longer periods of time as they age.

\section{Purpose of the Study}

The overall aims of this dissertation study were to investigate the effect of lighting on older adults' ability to perform selected occupational tasks, which require near vision. Lighting affects performance during reading and numeric tasks, especially in those with visual deficits (Bowers et al., 2001; Eldred, 1992; Fosse \& Valberg, 2004; Haymes \& Lee, 2006); however, much less is known about the effects of lighting during the performance of everyday tasks in people with aging vision. The literature also lacks studies that address the effort expended when performing tasks under different lighting levels. Consequently, the purpose of the dissertation study was to determine if lighting affects older adults' ability to perform selected occupational tasks, which require near vision and if lighting levels affects the perceived efforts of participants as they performed the selected occupational tasks.

\section{Research Questions and Hypotheses}

The research questions were the following: 
1. What is the impact of lighting on older adults' performance on selected tasks that are visually challenging?

2. What is the impact of lighting on perceived effort of older adults while performing tasks that are visually challenging?

The null hypotheses were the following:

1. Changing light levels will result in no significant changes in older adults' performance of visually challenging tasks as measured by time to complete tasks, where $\mu$ represents performance at each of the three lighting levels $\mathrm{H}_{0}: \mu_{1}=\mu_{2}=\mu_{3}$

2. Changing light levels will result in no significant changes in perceived effort of older adults while performing visually challenging tasks as measured on a self-report scale, where $\mu$ represents perceived effort at each of the three lighting levels.

$\mathrm{H}_{0}: \mu_{1}=\mu_{2}=\mu_{3}$

The research hypotheses for the study were the following:

1. Changing light levels will result in at least one significant change in task performance on visually challenging tasks as measured by time to complete tasks.

$\mathrm{H}_{\mathrm{A}}$ : at least two means will be significantly different

2. Changing light levels will result in at least one significant change in perceived effort while performing visually challenging tasks as measured on a self-report scale.

$\mathrm{H}_{\mathrm{A}}$ : at least two means will be significantly different 


\section{Definitions of Variables}

1. Cognition. Cognition is "cognitive mental processes" ("Cognition," n.d.), and "cognitive" is "of, relating to, being or involving conscious intellectual activity" ("Cognition," n.d.). Active engagement in activities that are cognitively challenging is important to maintaining and enhancing cognitive function, a concept that aligns with occupational therapy philosophy (Robnett, 2008). Cognition was operationalized and measured by scores on the MiniMental State Examination, Second Edition Brief Version (MMSE-2 BV; PAR, Inc., 2012).

2. Contrast sensitivity. Contrast sensitivity is "the capacity to distinguish between similar shades of light and dark and to distinguish similar colors" (Mogk, 2011, p. 29). It enables one to detect an object as the contrast between the object and its background diminishes, and it is very important to occupational performance (Meyers \& Wilcox, 2011). Contrast sensitivity was operationalized and measured through visual acuity scores on the Colenbrander mixed contrast card set (Precision Vision, n.d.).

3. Effort. Effort is "work done by the mind or body; energy used to do something" ("Effort," n.d.). Effort is as an invisible, internal, and hypothetical construct that cannot be directly observed (Yeo \& Neal, 2004). Effort was operationalized and measured through participants' self-rated scores on the Rating Scale Mental Effort (RSME; Zijlstra, 1993).

4. Occupational performance. Occupational performance is "the outcome of the transaction of the person, environment, and occupation. It is defined as the 
dynamic experience of a person engaged in purposeful activities and tasks within the environment" (Law et al., 1996, p. 16). A task is defined as work related to the development of occupational performance skills or a group of activities related to the accomplishment of a specific goal (Jacobs \& Simon, 2015). In the dissertation study, the investigator chose to utilize the term occupational tasks in reference to the activities participants were asked to perform. According to the OTPF (American Occupational Therapy Association [AOTA], 2014), the specific occupational tasks in this dissertation study were instrumental activities of daily living and included reading the label on a prescription bottle and sorting pills, which are classified as the occupation of health management and maintenance and sorting colored socks, which is classified as home establishment and management.

Occupational performance was operationalized through completion of the occupational tasks of reading a prescription label, sorting pills, sorting dark colored socks, and the time required for completing these occupational tasks.

5. Visual acuity. Visual acuity is "the level of detail with which a person can see objects" (Mogk, 2011, p. 29). Visual acuity was operationalized through visual acuity scores on the Colenbrander mixed contrast card set (Precision Vision, n.d.).

\section{Rationale and Need for the Study}

This dissertation study has important implications for occupational therapy practice because lighting affects performance in those with and without visual impairment (Bowers et al., 2001; Davis \& Garza, 2002; Eldred, 1992; Fosse \& Valberg, 
2004; Haymes \& Lee, 2006; Izso, Lang, et al., 2009; Petrofsky \& Cuneo, 2008).

Lighting has been shown to affect performance for reading and numeric tests (Bowers et al., 2001; Eldred, 1992; Eperjesi et al., 2006; Fosse \& Valberg, 2004; Haymes \& Lee, 2006; Izso, Lang, et al., 2009; Izso, Laufer, et al., 2009). However, there are very limited studies regarding the effect of lighting on the performance of daily living skills (Charness \& Dijkstra, 1999; Evans, Sawyer, Jessa, Brodrick, \& Slater, 2010), and the topic has not been addressed in occupational therapy research. The Hawthorne studies, conducted in the 1920s, began with an investigation of the relationship between illumination and productivity in plant workers and initially determined that performance was not affected as long as illumination was reasonable. However, the studies were later criticized when it was determined a number of variables could have been responsible for the increased productivity observed in the workers (Wickstrom \& Bendix, 2000). In more recent years, many researchers have investigated the effect of lighting on performance, utilizing participants with visual impairments (Bowers et al., 2001; Eldred, 1992; Eperjesi et al., 2006; Fosse \& Valberg, 2004; Haymes \& Lee, 2006), but only a few researchers have addressed lighting with age-related visual changes (Davis \& Garza, 2002; Izso, Lang, et al., 2009; Izso, Laufer, et al., 2009; Petrofsky \& Cuneo, 2008).

In addition, lighting in homes, an environmental setting where occupational performance occurs and where older adults spend so much time (Horgas et al., 1998; Kolanowski, 1992), has been found to be less than optimal (Bodart, Roisin, Deneyer, \& D’Herdt, 2009; Charness \& Dijkstra, 1999; Cullinan et al., 1979; Lindner et al., 2001; Simpson \& Tarrant, 1983), yet Perlmutter (2012) found that very few occupational therapists address lighting in the home environment. Furthermore, Krusen (2010) 
suggested occupational therapists need to learn about lighting in order to provide recommendations that will enhance occupational performance.

Information regarding the effect of lighting on occupational performance has societal implications as it will contribute to the knowledge base and plans for aging in place, which is the goal and desire of many adults (Gitlin, 2003; Gitlin et al., 2001). One's physical environment context includes accessibility as well as performance within the environment. Consequently, accessibility is determined by assessing the physical environment and one's functional performance within the environment. An important aspect of functioning within the environment is its usability or how easy one feels it is to function within the environment and the extent to which the environment supports or inhibits performance (Fange \& Iwarsson, 2005; Talley, 2008).

Environmental factors either facilitate or inhibit participation in daily occupations. The environment makes certain demands on a person's occupational performance (Hamilton, 2010), and these demands become increasingly more significant as a person ages (Haak, Ivanoff, Fange, Sixsmith, \& Iwarsson, 2007). Environmental barriers in the home often threaten the ability of older adults to remain in their homes as they age (Talley, 2008). These barriers occur when older adults experience decreasing functional abilities as a result of the aging process (Gill et al., 1999). Poor lighting affects ambulation in older adults (Kuyk \& Elliott, 1999; Reed et al., 2006) and poses a fall risk within the home (Tideiksaar, 2003; Woodland \& Hobson, 2003). Additionally, poor lighting has implications for older adults as they attempt to perform the everyday tasks of life, such as matching clothing (Barstow et al., 2011; Brunnstrom et al., 2004; Cook, 2009; Owsley et al., 2001) and managing medications (Henriques et al., 2012; Lam 
et al., 2011; MacLaughlin et al., 2005; Owsley et al., 2001; Skomrock \& Richardson, 2010; Windham et al., 2005; Yasein et al., 2013).

Because lighting in homes is designed with younger people in mind (Young, 2012), lighting in the homes of older adults must be addressed by occupational therapists during aging in place evaluations, and we must work with architects, interior designers, lighting engineers, and contractors to provide lighting that enhances performance within the home. This dissertation study addressed usability of lighting within the environment, drawing attention to the importance of lighting to occupational performance, an area that has received very little attention in occupational therapy literature.

\section{Theoretical Context}

The theoretical context for this study was the Person-Environment-Occupation (PEO) model. The PEO model conceptualizes a transactional approach between a person, the environment, and occupation with the product being occupational performance. The model identifies a person as a unique, holistic individual who possesses certain intrinsic qualities (physical, emotional, cognitive, and spiritual) as well as skills, abilities, and life experiences that influence engagement in occupational performance. Environment is broadly defined by the model, giving equal consideration to cultural, socio-economic, institutional, physical and social factors. The environment is where individuals engage in occupations and is influential in shaping behavior. Occupation is described by the model as tasks and activities in which a person engages, and all persons have an innate need to participate in occupation. Graphically, the three components, person, environment and occupation, are represented by inter-related circles, 
and the area in which the circles overlap represents occupational performance (see Appendix A; Law et al., 1996; Law \& Dunbar, 2007).

The PEO model separates occupation from the performance of occupation and acknowledges that a change in person, environment, or occupation affects occupational performance. The three components continually interact, and congruence among the three results in optimal occupational performance. Therefore, occupational therapy interventions may be directed towards the person, environment, or occupation with the goal of improving occupational performance (Ludwig, 2004).

McColl and Pollock (2005) asserted there are objective and subjective components to occupational performance. The objective, observable components are the behaviors associated with occupation, and the subjective, experiential components are the cognitive and affective experiences associated with the behaviors. Both components are essential to understanding and measuring occupational performance.

The development of the PEO model was heavily influenced by Lawton's ecological model, which describes the need for a just right fit between a person and the environment. When a person's functional ability (competency) and the demands of the environment (environmental press) are matched, the result is a just right challenge, adaptive behavior, and a positive effect. However, a mismatch between competency and environmental press results in maladaptive behavior and a negative effect. Consequently, the goal is to achieve a fit between a person's competency and environmental press (Rigby \& Letts, 2004; Sabata, 2008).

While Lawton recognized the importance of person-environment fit, Csikszentmihalyi (1997) and Coppola (2008) emphasized the importance of a fit between 
a person and the activity. Described as flow, this fit occurs when there is a match between an individual's skills and the challenge provided by the activity in which the individual engages. A better fit results in a more positive experience for the individual, whereas an experience that is too challenging or not challenging enough results in a less pleasurable experience. A person experiencing flow is immersed in the activity to the extent of losing awareness of self, time, and place (Coppola, 2008; Rigby \& Letts, 2004).

Although Lawton's person-environment fit and Csikszentmihalyi’s (1997) person-activity fit were influential in the development of the PEO model (Coppola, 2008; Rigby \& Letts, 2004) neither model acknowledges the transactional approach described in the PEO model, which depicts person, environment, and occupation as so closely associated that they cannot be teased apart. A complex model, the PEO model proposes that interactions can occur between the person and the environment, the environment and occupation, the person and occupation and the person, the environment and occupation, and the end result of these interactions is occupational performance (Cooper et al., 2005; Rigby \& Letts, 2004).

Unlike other disciplines, occupational therapy recognizes the importance of an individual's roles and the meaningful activities (occupations) an individual wants and needs to do as vital to understanding the person-environment relationship (Cooper et al., 2005; Rigby \& Letts, 2004; Sabata, 2008). Likewise, occupational therapists recognize the environment as the context for occupational performance (Cooper et al., 2005; Rigby and Letts, 2004) and understand that the environment can facilitate or inhibit occupational performance (Rigby \& Letts, 2004; Sabata, 2008). At times, there is a difference in an individual's capacity to perform meaningful occupations and his/her 
actual performance of those occupations (Cooper et al., 2005; Rigby \& Letts, 2004) due to environmental demands (press), which shape an individual's response (Rigby \& Letts, 2004; Sabata, 2008; Stark \& Sanford, 2005). When congruence exists between the factors a person brings to a transaction and the environmental demands, a fit occurs. However, when impairment couples with the environment and a person cannot do the required activities, disability results (Stark \& Sanford, 2005).

While environmental barriers can diminish the PEO model fit and occupational performance (Cooper et al., 2005), impairment may not be disabling if an individual is in an environment that supports performance (Stark \& Sanford, 2005). Oftentimes, the environment can be more easily changed than the person (Cooper et al., 2005); therefore, modifying the environment becomes important (Stark \& Sanford, 2005) and can be a cost effective means of supporting older adults in the community. Consequently, occupational therapists can play a vital role in assessing the environment to recommend necessary changes with the ultimate goal being a sound fit between the person, environment, and occupation in order to optimize occupational performance (Cooper et al., 2005; Rigby \& Letts, 2004).

The PEO model was selected for this dissertation study because it addressed the person (older adult), environment (lighting), and occupation (various occupational tasks). The congruence between the participant (person) as he or she performs various tasks (occupations) under different lighting levels (environment) defines the participant's occupational performance. The quality of the participant's occupational experience as defined by functional ability and satisfaction with performance is a direct result of the fit within the person-environment-occupation transaction (Strong et al., 1999). 
In terms of this dissertation study, intervention focused on changing the environment, specifically the lighting levels, in which occupational performance occurred. The objective aspect of behavior measured was the time required to perform different occupational tasks under the different lighting levels, and the subjective measure was the perceived effort while performing under the different lighting levels.

\section{Assumptions and Limitations}

\section{Assumptions}

Assumptions of the study were the following:

1. Participants put forth maximum effort during all occupational tasks.

2. Participants provided honest answers when rating effort.

3. The RSME (Zijlstra, 1993) measured effort.

4. The occupational tasks represented occupations that are pertinent to aging adults.

5. Because separate testing materials were used for the practice session and each of the three trials, participant status reverted to baseline after each trial, and no cumulative effect occurred after each intervention.

Delimitations of the study were the following:

1. Participants were delimited to those who were residents of the retirement community.

2. Participants were delimited to those who were 65 years of age or older.

3. Participants were delimited to those who volunteered to participate.

4. Participants were delimited to those with minimal vision loss or better with corrective lenses if worn. 
5. Participants were delimited to those who met minimal cognitive criteria.

6. The study was delimited to the three occupational tasks: reading a prescription label, sorting pills, and sorting dark socks. These tasks were chosen based on a review of the literature because they are gender neutral, functional tasks that require near vision and could be measured based on completion time.

7. The study was delimited to three average lighting levels: $100 \mathrm{fc}, 125 \mathrm{fc}$, and $400 \mathrm{fc}$.

8. The study was delimited to a rating scale to quantify effort because the investigator desired an objective means of measuring participants' subjective experiences.

9. The study was delimited to a repeated measures design because it was conducive to the population and occupational tasks being studied. The design allowed the participants to participate in the study in one visit, controlling for attrition and eliminating the need for follow-up visits and the possibility of physiological or cognitive changes during the course of the study. Also, the design allowed participants to serve as their own controls, eliminating the need for a control group in which participants might have compared information and perceived differences in the treatment that was provided to participants, an important consideration due to the close-knit nature of the community in which the participants reside.

\section{Limitations}

Limitations of the study were the following: 
1. A limitation of the study was the small sample size. The investigator placed flyers in 193 in-house mailboxes of residents. Thirty-seven residents volunteered to participate in the study. However, 31 residents kept appointments to participate in the study; one of those residents who volunteered did not meet the inclusion criteria. Therefore, the sample size consisted of 30 participants.

2. The weather was perceived as another limitation of the study. The investigator collected data between January 2015 and March 2015. To access the testing room utilized in the study, the investigator had to drive to the retirement community and participants had to travel outdoors via car or on foot from their place of residence in the retirement community to the Commons Building where the testing room was located. During this time period, there were several days of inclement weather that prevented the investigator and the residents from accessing the testing room. While 37 residents initially agreed to participate, six did not keep those appointments. The investigator had originally planned for the data collection period to take a few weeks. However, because of weather delays, data collection required a couple of months. Some of the residents who originally agreed to participate during the sign-up meeting in December were no longer willing or able to participate several weeks later.

3. Due to the study utilizing a sample of convenience, generalizability of the results was a limitation. The study was conducted within a retirement community in a large metropolitan area in Arkansas. The retirement 
community is owned and operated by a large not-for-profit health care system, and residents pay to live within the community. Further, the study was limited to those within the retirement community who volunteered to participate.

4. Random assignment of participants to groups prevented even numbers of participants within each group. The order of lighting levels was randomized as was assignment of participants to groups because the investigator did not want to risk any bias that might be incurred from participants communicating with future participants regarding a predictable format to the lighting levels, such as each participant experiencing low, medium, and then high light levels. Therefore, participants randomly selected a card that indicated only to the investigator the order in which light levels were presented. Because the investigator did not know how many residents would participate, there was not an equal number of participants in each of the groups.

5. Another limitation of the study was the repeated measures application that potentially led to testing effects as participants learned to anticipate the testing with each subsequent trial. The investigator designed similar testing kits in an effort to prevent differences in performance being attributed to the difference in testing kits. However, the kits may have been so similar as to contribute to a learning effect experienced by the participants.

6. Self-reporting by participants was another limitation of the study. Participants were asked to report demographic information, which required them to recall information, such as the presence of eye disease and the occurrence of falls, and they were asked to record their level of perceived effort when completing 
the occupational tasks. In acquiring this information, the investigator was relying on participants to accurately recall and honestly report such information.

7. Finally, because participants were made aware the investigator was conducting the research as part of her doctoral studies, they potentially could have provided information they felt would have benefited the investigator and the study. 


\section{Chapter 2: Review of the Literature}

\section{Overview}

This chapter provides an overview of the literature concerning lighting and task performance in individuals with and without visual impairment, lighting in home environments, and effort followed by a critique of the existing literature on these topics. Although this dissertation study included participants with aging vision, studies investigating aging vision are limited. Therefore, a review of the literature investigating the effect of lighting on individuals with low vision is included as information in those studies has implications for the study of aging vision. Also, the literature about home lighting and older adults' performance under home lighting as well as their perceptions of home lighting conditions demonstrate the need for further studies on this topic.

\section{The Effects of Lighting on Individuals with Low Vision}

Much of the research investigating lighting and performance has involved individuals with visual impairment, assessing reading performance in participants with age-related macular degeneration (ARMD; Bowers et al., 2001; Eldred, 1992; Eperjesi et al., 2006; Fosse \& Valberg, 2004; Haymes \& Lee, 2006). The effect of different levels of intensity of light as well as other characteristics of light, such as spectral power distribution and color temperature, have been examined. Eldred (1992) found lighting preference varied in adults with age-related maculopathy (AMD). Eighteen participants, aged 59 to 97 years of age, performed simulated reading tasks using the Pepper Visual Skills for Reading Test. Reading performance, measured in words per minute, was evaluated at six different light levels, adjusted by varying the distance between the task and the light source, two gooseneck lamps with 60 watt bulbs. The study was performed 
in an optometric examination room with an illumination level of 484.2 lux, a level the IESNA deemed appropriate for reading. There was no significant difference in reading performance (mean words per minute) between each illumination level, leading the researcher to conclude that optimum lighting levels are individually specific. However, it was noted that most participants performed better under higher lighting levels as 11 of 18 participants read the highest words per minute at illumination levels between 5,918 and 7,532 lux.

In a similar study, Bowers et al. (2001) also measured reading performance of 20 individuals, 60 to 85 years of age, with ARMD at six different light levels. Reading performance was evaluated by having participants read sentences of varying print size from the MNRead assessment. Print size, measured in logarithm of the minimum angle of resolution $(\log \mathrm{MAR})$ and presented in random order, ranged in size from $6 \mathrm{M}$ to 0.5 M. Conducted in an office setting, six illumination levels, ranging from 50 lux to 5,000 lux, were achieved utilizing dimmer switches for lower illumination levels and supplemental overhead lighting for higher illumination levels. At each lighting level, participants were asked if the light caused visual discomfort. Optimal illuminance level was determined to be the level at which the fastest reading rate and either the smallest critical print size (smallest print size at which the participant could read at maximum speed) or smallest threshold print size (smallest size at which the participant could read words) occurred. In $70 \%$ of participants, the objectively-determined optimal illumination was higher (median 3,500 lux) than the subjectively preferred level (median 2,450 lux). Researchers concluded that in individuals with ARMD, lighting levels should be 
determined individually, taking into consideration objective measures of performance and subjective measures such as visual comfort (Bowers et al., 2001).

Fosse and Valberg (2004) and Haymes and Lee (2006) found higher lighting levels improved reading performance in studies investigating the effects of lighting on reading speed in those with ARMD. Fosse and Valberg (2004) used the Tambartun Oral Reading Test to study the effects of luminance on oral reading speed in adults with ARMD aged 67 or older at a vision light laboratory in Norway. Six participants, aged 65 or older, with typical vision served as the control group. The level of luminance for optimal reading was determined using measures of visual acuity and self-reports, and critical print size was determined using the MNRead. Optimum magnification and print size were also determined for each participant as well. Participants with ARMD were tested at nine luminance levels ranging between $4 \mathrm{~cd} / \mathrm{m}^{2}$ and $1200 \mathrm{~cd} / \mathrm{m}^{2}$, and control group participants were evaluated at eight different luminance levels between $4 \mathrm{~cd} / \mathrm{m}^{2}$ and $1,200 \mathrm{~cd} / \mathrm{m}^{2}$. In participants with ARMD, reading rates varied and were dependent on lighting levels, and speed generally improved up to $80 \mathrm{~cd} / \mathrm{m}^{2}$. While reading rates for participants with ARMD were lower than the rates in participants with typical vision, the mean rate changed from approximately $28 \%$ of that of the control group at low lighting levels to $42 \%$ at the highest luminance levels.

Haymes and Lee (2006) studied the effects of task lighting, spectral power distribution (SPD), and illuminance on visual performance in 28 adults with ARMD (mean age of 78 years) and 18 age-matched adults with normal vision. Contrast sensitivity, reading speed, near visual acuity, color vision, and optimal print size were assessed utilizing four different task light sources (standard pearl incandescent lamp, 
daylight blue incandescent lamp, warm white fluorescent lamp, and cool white fluorescent lamp) at three illuminance levels between 300 lux and 3,000 lux. While there was a small but statistically significant $(p=.01)$ improvement in contrast sensitivity with the daylight incandescent lamp compared with the standard pearl incandescent lamp, Haymes and Lee (2006) found SPD did not significantly affect visual acuity, reading speed, print size, or color discrimination. Further, they found no significant differences in the effects of those with ARMD and those with normal vision. However, the results did indicate that increasing the illuminance level of task lighting had a significant effect $(p<.01)$ on visual function, particularly in those with ARMD. Near visual acuity and print size scores were significantly better at 3000 lux than 300 lux, particularly in those with ARMD.

Eperjesi et al. (2007) also examined performance utilizing four different lamps (standard clear incandescent, daylight blue incandescent, compact cool white fluorescent, and halogen incandescent) and determined reading performance was not affected by lights of varying spectral radiance (spectral power distribution). They investigated reading performance in 13 subjects with AMD and ARMD, utilizing four different types of task lighting and found no statistically significant difference in reading performance $(p$ $=.67 ; p=.74 ; p=.84)$ between the four types of lamps $(p=.78)$ with individuals with AMD or ARMD. Eperjesi et al. (2007) acknowledged that their results resembled the findings of Haymes and Lee (2006), and for future studies, they recommended determining each participant's optimal illuminance level and conducting tests at those levels. 
Cornelissen, Bootsma, and Kooijman (1995) compared object recognition in participants with low vision versus a control group of participants with normal vision, utilizing a "calibrated" visual environment that simulated a living room. Participants were placed in a dark room and asked to identify objects on the opposite end of the room. The lighting level in the room was gradually increased until the maximum level was reached or all the objects were identified. Cornelissen et al. concluded that increased light resulted in increased performance, and when those with normal vision reached maximum performance, those with visual impairment continued to improve in performance. However, they acknowledged that at higher lighting levels, some participants' performances diminished, leading them to conclude that lighting recommendations should be based on individual preference rather than visual status or diagnosis (Cornelissen et al. 1995).

Utilizing a repeated measures design, Evans et al. (2010) examined the effect of lighting on ADL performance in older adults with cataracts and ARMD. The 24 participants, aged 65 or older, were divided into three groups: cataracts, ARMD, and cataracts and ARMD. The groups were not matched for age or visual capability as the researchers were not aiming to make comparisons between groups. The goal was to search for significant relationships between lighting level and task performance within the participants in each group. Each participant carried out four different ADL tasks under three different lighting levels classified as dim (50 lux), medium (200 lux), and bright (800 lux); an additional task light was added for the reading task. The ADL tasks participants performed were walking along a corridor with an uneven floor (researcher observed from behind), inserting a plug into an electrical socket (repeated five times at 
each light level), sorting pills (20 round, white pills of varying sizes) and reading (Wilkins Rate of Reading test). These particular tasks were chosen because they had been utilized in previous studies (inserting a plug and reading) or were identified by the researchers after discussion with and observation of elders with poor vision. The researcher recorded the number of errors during task performance, and at the conclusion of each task, the participants were asked to rank which lighting level they found easiest and which they found most difficult. When discussing the results, the researchers defined significance at $p<.05$, and they defined a significance level of $.05<p<.10$ as approaching significance. In general, participants performed better under brighter light, but the results were individualized. For the walking task, in the cataract group and the ARMD group, brighter illumination was associated with faster performance. In the cataract group, repeated measures ANOVA demonstrated that the tendency for the three lighting conditions to differ approached significance $(F=2.70, p=.092)$, and in pairwise comparisons in the cataract group, the difference between dim and bright lighting $(p=$ $.056)$ and the difference between medium and bright lighting $(p=.085)$ approached significance. The difference between conditions did not reach significance in the ARMD group $(F=1.61, p=.25)$ or the combined cataract and ARMD group $(F=.160, p=.85)$. Overall, there was little effect for the task of inserting the plug in the socket. Repeated measures ANOVA showed no significant difference between conditions for the cataract group $(F=0.611, p=.55)$ or the combined groups $(F=0.57, p=.58)$. However, in the ARMD group, the difference among the three lighting conditions was significant $(F=$ $6.22, p=.018)$, and Evans et al. (2010) reported the adjusted pairwise comparisons were indicative of a significant difference between dim and medium lighting $(p=.047)$ and 
between dim and bright lighting $(p=.055)$. For the pill sorting task, in the cataract group, repeated measures ANOVA demonstrated lighting levels were significant $(F=$ $5.06, p=.017)$, and the adjusted pairwise comparisons indicated significant differences between dim and medium lighting $(p=.034)$ and between the medium and bright conditions $(p=.0008)$. The fastest performance occurred under the medium lighting level. The differences between conditions was not statistically significant for the ARMD group $(F=3.06, p=.14)$ or the combined group $(F=0.42, p=.67)$. For the reading task, the repeated measures ANOVA showed a significant effect of lighting condition $(F=$ $4.22, p=.03)$. The adjusted pairwise comparison indicated the difference between dim and medium lighting approached significance $(p=.082)$ and the difference between dim and bright lighting was significant $(p=0.033)$, but the difference between medium and bright light was not significant $(p=0.249$ ). The difference between conditions was not significant in the ARMD group $(F=2.01, p=.19)$. The difference between conditions approached significance in the combined cataract and ARMD group $(F=2.91, p=.10)$, and pairwise comparison between the dim and medium lighting conditions also approached significance $(p=.084)$. Regarding subjective preference, the brightest lighting level was typically perceived as the best and the dimmest was rated as the worst across all three groups and all tasks. Evans et al. (2010) concluded lighting is very individualized. They recommended further studies in testing situations in which lighting can be varied in order to find optimal levels. Further, they recommend gathering objective and subjective data regarding performance and preference. 


\section{The Effects of Lighting on Individuals with Normal Vision}

Petrofsky and Cuneo (2008) addressed the effect of lighting on performance in those with normal vision and conducted an investigation of the impact of lighting on balance skills. Izso, Lang, et al. (2009) and Izso, Laufer, et al. (2009) investigated an adaptive home lighting system. Davis and Garza (2002) investigated the impact of lighting variables on reading performance.

Investigating the effects of lighting on functional performance, Petrofsky and Cuneo (2008) found older adults exhibited more difficulty with balance skills than younger adults in dim or no light conditions. Under conditions of full light, dim light (5 candle power), and no light, balance and gait of fourteen young participants (ages 18-30) and 15 older participants (ages 40-68) were assessed. A custom-built, computerized, dynamic posturography device was utilized to assess balance during standing and reaching, and gait was assessed during initiation of movement, during walking and during turns, using an accelerometer.

Full light was not defined by the researchers, and the no-light condition was achieved by having participants close their eyes. Visual function of participants was not addressed. Petrofsky and Cuneo (2008) found that in static standing for a 30-second period, older adults experienced greater postural sway than younger participants under all three light conditions, and the degree of sway was significant at the no-light (ANOVA $p$ $<.01)$ and dim-light conditions $(p<.05)$. During the reaching task, older participants demonstrated significantly less shift in body weight under the dim light condition (ANOVA $p<.01$ ), and in comparing the older and younger participants, there was a significant difference between the two groups with all three light conditions (ANOVA $p$ 
$<.01)$. Gait was analyzed by comparing initiation of gait, continuous gait, and turns during gait. Older participants demonstrated a greater stance/swing ratio under all light conditions, and the velocity of their gait was slower under all conditions. When comparing numbers of steps in the older group, the numbers of steps were significantly greater in dim light compared to full light $(p<.05)$. Additionally, older adults experienced significantly greater movement in their ankles $(p<.01)$ and greater circumduction $(p<.05)$ during dim versus normal light. Petrofsky and Cuneo (2008) proposed that visual changes due to aging, including control of eye movement and color vision, affect an older adult's ability to distinguish environmental borders, such as the edge of objects, and these changes cause them to inaccurately assess their body position in space. Further, because gait involves multiple body systems, including visual, vestibular, somatosensory, and motor systems, an older adult might receive false cues in dim lighting conditions, thereby overloading his/her system and causing abnormalities in gait in an effort to compensate for balance.

For the purpose of developing an adaptive home lighting system for the elderly, Izso, Lang, et al. (2009) and Izso, Laufer, et al. (2009) investigated performance and subjective feelings of older adults under different lighting conditions and found significant performance differences under the different lighting conditions. Thirty adults 65 years of age and older with normal vision participated in a variety of tasks, including reading, completing a numerical verification test (NVT), watching calm/exciting video, relaxing (eyes closed/open), sitting, and standing. The tasks were completed under the following lighting conditions: own (participant selected from variety of available lamps; range from 100 lux to 1,500 lux; mean lux $=412$ ), normal (standard lighting device 
measuring $~ 1,300$ lux on the desk), relaxing (standard lighting device measuring $~ 100$ lux on the floor), and strong (standard lighting device measuring 5,000 lux on the desk), but the results of the own lighting levels were omitted from the comparisons in the study because the levels were different for each participant. While performing tasks under the different light levels, participants were evaluated in three areas: psychophysiological correlates (heart rate, skin conductivity, respiration, and electromyogram activity), performance correlate (NVT), and subjective correlates. The correlates included a sixpoint semantic differential scale that asked participants to rate their perception of lighting. Examples of categories included pleasant/unpleasant, relaxing/activating, bright/dark, and like/did not like. Skin conductivity was the psychophysiological measure that was most sensitive to differences in activity and lighting. Additionally, participants performed more accurately under normal lighting levels rather than relaxing or strong lighting levels as indicated by the NVT results. Finally, low color temperature $(2,700 \mathrm{~K})$, low illumination ( 100 lux), and relaxing lighting was subjectively rated as relaxing. Medium color temperature $(4,000 \mathrm{~K})$, medium illumination $(\sim 1,300 \mathrm{lux})$, and normal lighting yielded the best, most accurate results with mentally challenging tasks, such as the NVT.

Izso, Laufer, et al. (2009) studied the effects of two dynamic lighting conditions on the performance and subjective feelings of 12 older adults. The participants were greater than 65 years of age and had normal or corrected-to-normal vision. They were asked to perform a computerized version of the NVT for a total of 32 minutes with a four-minute break in the middle as lighting changed between levels of 300 lux to 900 lux. Speed and accuracy of the tasks were rated, and additionally, participants were asked to 
rate their feelings of "relaxation" and "pleasantness" on a subjective scale. Izso, Laufer, et al. (2009) hypothesized the change in lighting would affect performance on the NVT and that the brighter light would be preferred and lead to increased performance. However, as a group, there was no statistically significant difference in performance between the two levels of lighting. The only statistically significant difference was that the standard deviation of hit number on the NVT was higher during the up-lighting condition than during the down lighting condition. However, participants did subjectively rate the brighter light as more pleasant, leading Izso, Laufer, et al. (2009) to conclude that individual consideration should be considered in lighting design.

At an independent living center in Colorado, Davis and Garza (2002) investigated the effects of four different lighting variables (illuminance, uniformity distribution, correlated color temperature, and surrounding reflectance or background) at three different light levels ( $5 \mathrm{fc}, 30 \mathrm{fc}$, and $120 \mathrm{fc}$ ) on reading performance for 17 participants, 62 to 76 years of age and free of documented eye disease. Using four independent variables yielded 36 possible testing conditions, and of those, 17 were selected for the study. Variables addressed included illumination, background, and uniformity. As illumination increased, NVT scores increased, and a significant difference was found among all three illumination levels. Further, NVT scores increased as light increased for all levels of uniformity, background, and CCT. Illumination was also significant on all subjective measures. Also, in subjective measures, a black background was preferred, and the difference between backgrounds was greatest at the highest illumination levels and with uniform distribution. However, background did not affect performance. No significant results were found regarding uniformity of light, but the trends were similar to 
those found with background. Izso, Laufer, et al. (2009) concluded that illuminance of greater than $100 \mathrm{fc}$ yielded the greatest visual performance and was the most preferred by participants.

\section{Studies of Lighting in the Home}

Lighting conditions in the home and the effect of lighting on performance in the home environment have been evaluated in a limited number of studies, and a few of those studies will be discussed in this review (Bakker et al., 2004; Bodart et al., 2009;

Brunnstrom et al., 2004; Charness \& Dijkstra, 1999; Lindner, et al., 2001; Simpson \& Tarrant, 1983; Soressen \& Brunnstrom, 1995). In adults with low vision, Brunnstrom et al. (2004) investigated the effect of lighting on the performance of ADLs before and after lighting adjustments were made in the home. Utilizing a pretest-posttest control group design, lighting changes were made in the kitchen, hall, and bathroom of all participants, and those in the intervention group also received lighting changes in the living area as well. Follow-up interviews indicated the lighting changes in the living area noticeably improved the quality of life of those in the intervention group. Quality of life was assessed via interview questions and a psychological and general well-being scale (PGWB). Interview questions addressed factors, such as loneliness and self-esteem, whereas the PGWB scale assessed the dimensions of well-being, vitality, and depression. Both the interview and the PGWB scale asked participants to rate factors on a scale from 1 (worst perception) to 7 (best possible perception). A total of 11 factors were assessed via the interview and PGWB scale, and all were found to be statistically significant $(p<$ $.05)$. 
Lighting levels in home environments tend to be lower than recommended industry standards (Bodart et al., 2009; Charness \& Dijkstra, 1999; Cullinan et al., 1979; Lindner et al., 2001; Simpson \& Tarrant, 1983). Bodart et al. (2009) conducted a lighting renovation project in Belgium, applying different lighting systems and lighting levels to six similar apartments. They replaced incandescent bulbs with more energy efficient bulbs in the apartments and found that while the bulbs were more energy efficient, they did not always result in adequate lighting that met industry standards. Moreover, they determined that when residents were allowed to decide lighting installation, the lighting power was low and did not meet targeted levels. However, when residents were surveyed about lighting conditions via questionnaire, responses were generally positive.

Simpson and Tarrant (1983) studied lighting behavior in 101 randomly selected homes in Surrey, England. Fifty of the homes studied were owner occupied, and 51 homes were rented, and the study included a description of fixed and portable lighting fixtures. Lighting levels in the sitting room, kitchen, entry way, and stairway of each home were compared with levels recommended by the Chartered Institution of Building Services/Illuminating Engineering Society (CIBS/IES) code. Measurements were taken in the evenings in order to measure only artificial light. A purpose of the study was to determine whether factors, such as age, social class, income, and ownership versus renting affected occupants' use of and attitude toward lighting, and Simpson and Tarrant (1983) concluded that home owners were more likely to make changes to lighting. However, several other findings were worthy of discussion. In most homes, illuminance levels were inadequate, and the inadequacy was attributed to poor provision of fixed lighting in the homes. In the kitchen, most homes had less than the CIBS/IES code 
recommendation of 300 lux, and most homes had only one ceiling lamp as the single light source in the kitchen. Often, the occupant's body cast a shadow over the work surface, which contributed to the low light levels at the work surface. Similarly, lighting at the bottom of staircases also fell below the recommended 100 lux level.

Simpson and Tarrant (1983) also investigated light levels in locations in which three different tasks were performed, primarily in the sitting or sitting/dining room. The tasks, chosen because they are identified in the CIBS/IES code, were sewing/darning, casual reading (newspaper, magazine, etc.), and prolonged or desk reading (small print books, accounts, etc.). Mean lighting levels for these tasks fell below the CIBS/IES recommended levels of 300 lux for sewing and prolonged reading and 150 lux for casual reading although lighting levels did tend to be higher for occupants who were over 40 years of age. Lighting provisions in the sitting room varied greatly, and while the majority of homes had more than one light source, $22 \%$ had only one light source. Simpson and Tarrant (1983) proposed that lighting in sitting rooms centers around appearance and effect, noting it is unlikely tasks in these areas will be well lit due to the limited number of light sources in the room. Further, they noted that when roughly half of participants were performing tasks, they were not near a light source. Simpson and Tarrant (1983) concluded that while some participants added portable lighting, most were content to leave the current lighting provisions in their homes. Also, they concluded that in areas in which accurate vision is essential, lighting was below standards recommended by CIBS/IES.

Lindner et al. (2001) studied the illumination levels in reading locations of 91 visually impaired people. Most participants read in a living room or kitchen, and $10 \%$ of 
these spaces had an illuminance level greater than 5,000 lux while 63\% had less than 500 lux. Incandescent lamps were the primary light source in the reading spaces, and $60 \%$ used the ceiling light as the light source for reading while only $40 \%$ of participants used an additional light source in the reading area.

In a multi-stage, longitudinal study, Sorensen and Brunnstrom (1995) examined the impact of lighting on quality of life in a group of randomly selected adults 65 years of age or older. In the first stage, participants completed a questionnaire pertaining to quality of life and lighting within the home. Two researchers examined lighting in the home, labeling it as "acceptable" or "poor." Participants whose lighting in the reading area of the living room was labeled as "poor" were selected to participate in the intervention study. Poor lighting in the reading area was identified in 48 homes. The 48 residents of the homes agreed to participate in the study and were assigned to the experimental or the control group. Participants in both groups participated in an interview and a reading test to determine optimal font size. Those in the experimental group were provided a floor lamp to be placed in the reading area. Follow-up interviews and reading tests were conducted at 3 months and at 12 months. Three years after the initial interviews, a follow-up questionnaire on quality of life was administered. The changes in lighting were found to have a significant effect on quality of life, and these changes persisted throughout the 3.5-year period of the study.

In a descriptive pilot study, Bakker et al. (2004) measured lighting levels in the homes of 40 homebound adults aged 65 and older with and without self-reported visual impairment, residing in Manhattan, New York. Participants were asked to complete a questionnaire regarding their daily activities and lighting usage preferences. A lighting 
inventory and measurement of lighting levels of ambient and task lighting were taken in selected locations throughout each home. For example, participants were asked to read instructions on a pill bottle in the location where they typically took their medication, utilizing the light level they normally used. When compared with IESNA lighting standards, nearly all homes had inadequate ambient and task lighting, yet participants rated the lighting as adequate. Bakker et al. (2004) noted most participants relied on natural light rather than fixtures. Electric light was provided by table and floor lamps rather than ceiling or wall fixtures. Also limiting were non-working fixtures, dust covered bulbs, and dark lamp shades. Bakker et al. (2004) recommended further study to determine if recommended standards are optimal and feasible in homes.

Charness and Dijkstra (1999) studied the luminance level and legibility performance of 98 Florida residents in 81 homes. Participants, who ranged in age from 20 to 82 years of age, were divided into young $(20-38 ; N=31)$, middle-aged $(39-58 ; N=$ 33 ) and older (over age 58; $N=34$ ) age groups. Participants were tested in the morning and the evening and were asked to perform four tasks: read a newspaper, proofread digits under ambient light, proofread digits under a lamp, and locate a listing in a telephone book. Charness and Dijkstra found that while younger and older adults had home lighting levels that were inadequate for reading, older participants had higher lighting levels in their homes, particularly during the day. Nonetheless, these lighting levels were still below standard, and older adults performed less quickly than younger adults on most legibility tasks. However, the addition of lighting helped younger and older adults equally on most legibility tasks. Charness and Dijkstra expected older adults to benefit more from increased lighting; however, the benefits were about the same for younger and 
older participants. Charness and Dijkstra (1999) found that the benefits being about the same was because the normal lighting levels in the homes were still below recommended standards.

\section{Effort}

Effort is the energy utilized to do something ("Effort," n.d.) and may be physical or mental (Yeo \& Neal, 2008). Yeo and Neal (2004) described effort as an invisible, internal, and hypothetical construct that cannot be directly observed; is difficult to measure; and, consequently, is not often studied. Nevertheless, a few researchers have investigated effort required for various tasks, such as the effort required for listening (Fraser, Gagne, Alepins, \& Dubois, 2010), the mental effort expended during mobility (Geruschat \& Turano, 2007), effort expended in driving when following another vehicle (Lewis-Evans, De Waard, \& Brookhuis, 2010), and the relationship between facial expression and perceived effort during a physical task (De Morree \& Marcora, 2010).

Methods of quantifying mental effort can be classified in three broad categories:

1. Physiological indicators (i.e., heart rate and muscle activity).

2. Performance measures (on primary and secondary tasks).

3. Subjective measures (ratings of task load and questionnaires regarding the individual's state; Zijlstra, 1993).

Methods of quantifying effort have included evaluating an individual's physiological response during task performance, recording the time required to complete a task, and subjective self-report measures of the amount of effort expended when completing a task (de Morree \& Marcora, 2010; Fraser et al., 2010; Geruschat \& Turano, 2007; Lewis-Evans et al., 2010). Fraser et al. (2010) utilized a single question to ask 
participants how effortful it was to listen in a noisy environment, requiring participants to rank the effort utilized on a scale from 0\% (no effort) to 100\% (very effortful). Similarly, Lewis-Evan et al. (2010) asked participants to rate on a seven-point scale (1 for no effort and 7 for maximum effort) the effort expended in following a lead car at a specified distance. Geruschat and Turano (2007) utilized reaction time to a secondary task as an estimation of mental effort required when performing a primary task, and de Morree and Marcora (2010) utilized electromyelograms (EMG) of facial muscle activity to measure effort during a physical task, validating those results against participants' reports of effort on the Rate of Perceived Exertion (RPE), a self-report tool, which asks participants to utilize a numeric scale to indicate how effortful a task was.

With the exception of Geruschat and Turano's (2007) study, which utilized the Rate of Perceived Exertion, none of the aforementioned studies utilized a specific instrument to measure effort. The Rate of Perceived Exertion asks participants to rate the amount of physical effort expended during an activity and, consequently, does not measure effort in the manner desired in this dissertation study.

Zijlstra (1993) suggested that many existing tools that reportedly measure effort actually measure work load. He recognized a distinction between workload and effort, and in response to the need for an instrument that measures mental effort, he developed the RSME. Developed in the Netherlands, the RSME is a unidimensional scale that asks participants to indicate the amount of effort invested in a task by placing a mark along the scale. The RSME is a vertical axis scale ranging from 0 to $150 \mathrm{~mm}$ with every $10 \mathrm{~mm}$ indicated. At various points along the line, at certain anchor points, there are nine descriptive indicators ranging from 3 (not effortful) to 114 (awfully effortful). Individuals 
are asked to mark a point on the scale that is indicative of the mental effort exerted during task performance (de Waard, 1996; Wilson, Smith, \& Holmes, 2007). Since its development, the RSME has been utilized to measure the mental workload of drivers (de Waard, 1996), and Capa et al. (2008) utilized the scale when studying the effect of motivation to achieve and task effort on mental effort. The RSME was among the workload assessments in the driving environment that Verwey and Veltman (1996) compared in order to determine the sensitivity (extent to which it differentiates between different levels of a workload) and diagnosticity (the extent to which it responds differently to different types of workload) of the assessment. When comparing the RSME with another assessment, the Subjective Workload Assessment Technique (SWAT), Capa et al. (2008) concluded the RSME was simpler and somewhat more sensitive than the complex SWAT. Assessment of the reliability of the RSME indicated reliability of the scale in laboratory $(r=0.88)$ and real-life settings $(r=0.78)$ is acceptable. Additionally, the scale was found to correlate strongly with psychophysiological indicators of mental effort (Wilson et al., 2007). During the initial development of the RSME, the scale was utilized as part of a large study that investigated the workload behaviors of city bus drivers in the Netherlands. The workers were asked to rate their effort expenditure at various points throughout the day on both working and non-working days. Scores on the RSME were validated by comparing them against the actual increasing workload, reaction times of participants, and the physiological responses of participants (Zijlstra, 1993).

In her review of workload measures, Miller (2001) viewed the RSME as a scale that rates invested effort of the task rather than specifically mental effort. However, 
Zijlstra (1993) maintained while mental effort has been viewed as a construct that is unconscious and unavailable for conscious evaluation, it is a construct that can be measured, and the RSME is capable of measuring mental effort expended during task performance. Zijlstra noted that in recent years, attitudes toward subjective assessments have become more positive. He proposed that when measuring mental workload, subjective ratings may come closest to securing the essence of this construct. Further, he noted the subjective experience of an individual goes beyond the matter of the rating, emphasizing the importance of the phenomenological experience of the individual to the individual's overall performance. He proposed that during task performance, if an individual perceives the workload of a task is excessive, the individual may react as if he or she is overloaded even though the task demands are low. Consequently, the individual may employ behaviors, such as responding with less accuracy, spending less time on the task, or lowering the standards for acceptable performance of the task. He maintained the importance of distinguishing between workload, which is external and pertains to objective complexity of task demands, and the effort investment, which relates to the individual's performance potential and accounts for changes in the state of the individual, such as stressors that affect the individual's psychophysiological state, and affect work capacity. Importantly, Zijlstra (1993) acknowledged the value of the relationship between the workload of a task and the context of the task.

Zijlstra (1993) maintained that the scores of the RSME can be interpreted as the cost to an individual when performing a certain task under certain circumstances. He also acknowledged the relationship between effort and time, and maintained that during task performance, there is always a trade-off between the effort and time. When 
individuals feel they have ample time to perform a task, then they may perceive that less effort is exerted. However, if pressed for time, they may feel the need to exert more effort in order to complete the task in a timely manner. Hence, there is a reciprocal relationship between the two constructs. This relationship has important implications for the dissertation study. Because of the association between time and effort, both will be measured in the dissertation study.

\section{Critique of Theory}

A theoretical context is very important to any body of knowledge and research endeavor. Theories are useful in summarizing existing knowledge and inspiring the development of new knowledge. They provide a basis for the development of research questions, guide researchers in predicting outcomes, and are instrumental in interpreting results (Portney \& Watkins, 2009). Therefore, it is concerning that to date, studies addressing vision and performance lack a theoretical foundation. None of the studies in the literature review mentioned a theoretical perspective. This omission has been recognized, but as of yet, not addressed. Owsley (2011) noted that research about vision and aging would be most beneficial if it is driven by a theoretical framework and practical needs. From a much broader perspective, Gitlin (2003) also recognized the lack of theoretical direction in studies of the home environment. She urged researchers to examine specific person-environment characteristics and environmental strategies that contribute to living at home and quality of life.

\section{Summary of the Literature}

Researchers have demonstrated that lighting affects performance in individuals with and without visual impairment (Bowers et al., 2001; Davis \& Garza, 2002; Eldred, 
1992; Fosse \& Valberg, 2004; Haymes \& Lee, 2006; Izso, Lang, et al., 2009; Petrofsky \& Cuneo, 2008), but many of these studies have been conducted utilizing numeric and reading tests (Bowers et al., 2001; Eldred, 1992; Eperjesi et al., 2006; Fosse \& Valberg, 2004; Haymes \& Lee, 2006; Izso, Lang, et al., 2009) rather than the functional tasks individuals complete on a daily basis. Bakker et al. (2004) and Cook (2009) have investigated performance that measured participants' satisfaction with lighting, and if tasks were performed during the study, specific measurement procedures were not described. Also, the studies have been conducted by other disciplines, such as architecture, engineering, gerontology, home economics, and psychology rather than by occupational therapists.

Further, studies have identified concerns with lighting in home environments (Cullinan et al., 1979; Cumming et al., 1999; Gill et al., 1999; Greene et al., 2009; Iwarsson \& Isacsson, 1996), and light levels are often below recommended standards (Charness \& Dijkstra, 1999; Lindner et al., 2001; Simpson \& Tarrant, 1983). While poor lighting affects performance of everyday tasks, such as clothing management (Barstow et al., 2011; Brunnstrom et al., 2004; Cook, 2009; Owsley et al., 2001), it has also been shown to influence occupations, such as functional mobility (Kuyk \& Elliott, 1999; Reed et al., 2006; Tideiksaar, 2003; Woodland \& Hobson, 2003) that has the potential for serious consequences should errors occur. Nonetheless, older adults often rely on existing ambient lighting rather than adding to existing lighting. Additionally, they describe the lighting as adequate even when it is not (Bakker et al., 2004; Lindner et al., 2001). An investigation of the influence of lighting on the performance of functional 
tasks that older adults perform as part of their everyday ADL and IADL routines is lacking in the literature.

A few researchers have investigated effort required for various tasks, such as the effort required for listening (Fraser et al., 2010), the mental effort expended during mobility (Geruschat \& Turano, 2007), effort expended in driving when following another vehicle (Lewis-Evans et al., 2010), and the relationship between facial expression and perceived effort during a physical task (De Morree \& Marcora, 2010). Methods of quantifying effort have included evaluating an individual's physiological response during task performance, recording the time required to complete a task, and subjective selfreport measures of the amount of effort expended when completing a task (de Morree \& Marcora, 2010; Fraser et al., 2010; Geruschat \& Turano, 2007; Lewis-Evans et al., 2010). In response to the need for a tool measuring mental effort, Zijlstra (1993) developed the RSME, a unidimensional scale that asks participants to indicate the amount of invested effort into a task by placing a mark along the scale. The RSME is a vertical axis scale ranging from 0 to $150 \mathrm{~mm}$ with every $10 \mathrm{~mm}$ indicated.

Studies of functional performance and lighting have been descriptive in nature, relying on self-report measures of lighting behavior (Bakker et al., 2004; Cook, 2009) or seeking information, such as satisfaction with performance (Sorensen \& Brunnstrom, 1995) rather than emphasizing actual observed, measured performance of functional tasks under measured lighting conditions.

A measured performance of specific occupational tasks under measured lighting levels that meet IESNA standards has not been addressed. Further, while researchers have examined lighting preference and satisfaction with lighting (Bakker et al., 2004; 
Cook, 2009; Davis \& Garza, 2002; Sorensen \& Brunnstrom, 1995), none have evaluated participants' perceived efforts while performing tasks under different lighting levels. This dissertation study has served as a beginning point in examining the impact of lighting on performance of older adults, and it provides insight into the impact that lighting has on the effort exerted when performing a task. Investigating performance and perceived effort during performance captured both the objective and subjective aspects of occupation performance (McColl \& Pollock, 2005) and provided insight into the impact that environmental modifications have on the objective and subjective aspects of occupational performance. This information has potentially important implications for occupational therapy practice. Environmental modifications, such as changes in lighting, have the potential to improve occupational performance in older adults with aging vision (Cooper et al., 2005). Additionally, the study contributes to the occupational therapy literature, which has been relatively silent on the topic (Gendeman et al., 2010) and beyond, adding to the limited knowledge of the impact of lighting design on aging vision (Nylen et al., 2014). Furthermore, through use of the PEO model, this dissertation study provided a theoretical context, which is absent from studies of lighting and performance to date and contributes to an understanding of the impact of environmental modifications on occupational performance (Law et al., 1996). 


\section{Chapter 3: Methodology \\ Research Design and Methodology}

The investigator utilized a within-subjects repeated measures design with randomly assigned intervention order. Utilizing this design, each participant was tested under each condition (lighting level), acting as his/her own control. In so doing, the investigator believed the differences observed were more likely to indicate the difference in lighting levels rather than differences between participants (Portney \& Watkins, 2009). The $F$ test for repeated measures analysis of variance (RMANOVA) was run and assumptions were violated to the extent that the results were not tenable. Therefore, a three-way analysis of variance (ANOVA) was run, yielding the same result. Consequently, the research questions were addressed using descriptive statistics. This data will be further explained in the data analysis section.

\section{Rationale}

The investigator believed a repeated measures design with interventions completed in one session was an appropriate methodology for this dissertation study with this particular group of participants for several reasons. First, because each participant acted as his/her own control, extraneous variables, such as differences in age, gender, and vision variability between groups, remained constant, meaning differences observed were more likely due to the differences in lighting levels rather than individual differences among participants. Because of the nature of the lighting intervention, a cumulative effect was not a concern (Portney \& Watkins, 2009); however, the concern for the investigator with this group of participants was the potential for physiological or cognitive changes during the course of the study and the ability to have participants to return for additional 
sessions or complete the study. Additionally, having each participant serve as his/her own control allowed each participant to be tested under each of the three lighting levels and eliminated the need for a control group in which participants might have compared information and perceived differences in the treatment being provided to participants. This consideration was important for the investigator due to the close-knit nature of the community in which the participants resided. Finally, the within-subjects design was very efficient, requiring fewer subjects and less time to complete (Hall, 1998).

\section{Specific Procedures}

The investigator received written consent from the presiding vice president of the retirement community to recruit residents of the community and to utilize a room in the Commons Building on the grounds of the community. The investigator received approval from the Institutional Review Board (IRB) of Nova Southeastern University (NSU) to conduct the study, and this approval was shared with the facility's Director of Clinical Research and approved by the Clinical Research Committee. Participants were informed of the study via flyers placed throughout the Commons Building within the retirement community and in the 193 in-house mailboxes (see Appendix B). At the meeting, 37 residents signed up to participate in the study (see Appendix C). No additional residents contacted the investigator requesting to participate in the study. Thirty-one of the 37 residents kept their appointments and participated in the study. One of the residents who volunteered did not meet the inclusion criteria to participate in the study. Therefore, 30 participants completed the study. 
The nature of the study was thoroughly explained to participants, and they were asked to sign an informed consent form. Following the signing of informed consent, the following procedures were followed:

1. Steps 1 to 5 were completed under general ambient lighting in the testing center. Participants were instructed to wear corrective lenses if desired and if applicable. The ambient lighting in the testing center was provided by two ceiling-mounted overhead fixtures. Each fixture consisted of four Phillips F32 T8/TL741 700 series, 32 watt, Alto collection G6 fluorescent tubes. The fixtures were controlled by a single light switch located on the wall to the left of the doorway. The total ambient lighting in the room averaged approximately $103 \mathrm{fc}$.

2. Cognitive status was assessed with the Mini-Mental State Examination, Second Edition Brief Version (MMSE-2 BV; PAR, Inc., 2012).

3. Visual acuity and contrast sensitivity were assessed with the Colenbrander Mixed Contrast Card Set (Precision Vision, n.d.).

4. The participant who did not meet the inclusion criteria was thanked for her time and dismissed. Participants who met inclusion criteria were asked to continue in the study.

5. A demographic information sheet was completed (see Appendix D).

6. Participants drew a card indicating to the investigator the order of the lighting levels (see Appendix F). Lighting levels were adjusted by the investigator, according to the order indicated on the card drawn by each participant. 
7. Participants completed occupational tasks (contained in the testing sets) a total of four times as follows:

- A practice session with a Practice Testing Set under general ambient lighting.

- Complete the RSME.

- Trial 1 Testing Set \# 1 under lighting level

- Complete the RSME.

- Trial 2 Testing Set \# 2 under lighting level

- Complete the RSME.

- Trial 3 Testing Set \# 3 under lighting level

- Complete the RSME.

Prior to each trial, the investigator adjusted the lighting in the testing center according to the lighting levels indicated on the card and measured the lighting with a light meter to ensure the light was at the desired fc. The lighting level was measured by placing the light meter in the center of the work area on the desk; the lighting level was measured, and then the light meter was removed and the task was placed on the desk. As each occupational task was performed, the investigator timed participants utilizing a stopwatch and recorded completion time to the nearest hundredth of a second (see Appendix E). At the completion of the three occupational tasks, the investigator recorded the cumulative time required to complete the occupational tasks.

The lighting levels utilized the following:

- For the trial session, the general ambient lighting averaged $103 \mathrm{fc}$. 
○ The testing lighting levels utilized the following:

- Ambient lighting averaged $103 \mathrm{fc}$.

- Ambient lighting and floor lamp averaged $127 \mathrm{fc}$.

○ Ambient lighting, floor lamp and task lamp averaged $397 \mathrm{fc}$.

8. Participants were thanked for their time. Estimated participation time was 30 to 45 minutes.

Ambient lighting in the testing area was measured with a light meter and averaged $103 \mathrm{fc}$. Lighting was provided by two overhead fixtures with four fluorescent bulbs per fixture (F32 T8/TL741 700 series, 32-watt, Alto Collection G6) as well as with additional lighting that was adjusted throughout the procedures:

- A Hampton Bay floor lamp with a reading lamp, which was 71.5 inches tall and contained two bulbs at 1600 lumens (100-watt equivalent) with shade, which directed light toward the ceiling and an 800 lumens (60-watt equivalent) lamp with shade, which directed light downward.

- A Mainstays Swivel Organizer desk lamp, which was approximately 10 inches from base to light source and utilized an 800 lumens (60-watt equivalent) lamp with shade, which directed light toward the task.

Lamps (bulbs) utilized in the floor lamp and task lamp were as follows:

- EcoSmart 100-watt equivalent bright white CFL, 1,600 lumens, 3,500 K.

- EcoSmart 60-watt equivalent bright white CFL, 800 lumens, 3,500 K.

The floor lamp was placed to the right side of the participant (IESNA, 2010), and the task lamp was placed in front of the participant. The investigator sat to the left of each participant. 
In designing the lighting order, the investigator was interested in how participants would perform the occupational tasks when exposed to the lighting in order from low to high. Therefore, Card A indicated the order of lighting as low-medium-high. However, the investigator realized that in utilizing this lighting order in which the most intense lighting occurs on the final trial, changes in performance could be attributed to not only increasing light levels but also to a practice effect. The investigator was also interested in performance when participants were initially exposed to the highest level of lighting, and lighting was presented in descending order. Consequently, Card B indicated the order of lighting as high-medium-low. However, with lighting presented in this order, changes could be attributed to fatigue rather than a lower lighting level. Therefore, an additional consideration was how participants would perform if lighting was presented in a random pattern. For that reason, Card C indicated the order of lighting as medium-high-low (see Table 1).

Table 1

Lighting Order

\begin{tabular}{cccc}
\hline Group & \multicolumn{3}{c}{ Lighting level } \\
\hline & $\underline{\text { Trial 1 }}$ & $\underline{\text { Trial 2 }}$ & $\underline{\text { Trial 3 }}$ \\
Card A/Group 1 & Low & Medium & High \\
Card B/Group 2 & High & Medium & Low \\
Card C/Group 3 & Medium & High & Low \\
\hline
\end{tabular}

The investigator consulted with a statistician regarding the research design throughout the research process. However, in the proposal, the researcher's explanation of the lighting order did not clearly convey the rationale for the design of the lighting order in each of the groups as explained above. As a result, the weakness in the design of the groups was not detected until the data analysis phase of the study. The low lighting level is not represented in Trial 2, and the medium lighting level is not present in Trial 3 
(See Table 1). Consequently, the RMANOVA could not be completed as planned because each lighting level was not represented in each trial, and as a result, the investigator was unable to test the null hypotheses using the planned statistical procedures. Therefore, a three-way ANOVA (group x trial x light level) was calculated for the time required to complete each of the three occupational tasks (reading a prescription label, sorting pills, and sorting socks), the total completion time of all three tasks, and the RSME. Due to violation of the assumption of complete cells, the $F$ statistic could not be calculated for trial and light level, but it was calculated for group.

Therefore, the results of the ANOVA for group as well as the descriptive statistics were analyzed and utilized to address the research questions. This will be further explained in Chapter 4.

\section{Threats to Internal Validity}

Threats to internal validity associated with repeated measures design include testing/practice effect and instrumentation. The testing/practice effect threat was addressed by utilizing three separate but equal sets of testing materials, a different set at each lighting level. Instrumentation could have potentially served as a threat due to the variation in lighting that was utilized; however, the lighting level was monitored and recorded throughout the testing in order to ensure lighting levels remained within the established ranges. Natural daylight was not a factor as there were no windows in the testing room.

\section{Threats to External Validity}

Threats to external validity included the interaction of treatment and selection and the interaction of treatment and setting. Interaction of treatment and selection was 
controlled by beginning with one practice session to ensure participants understood the instructions and had the opportunity to ask questions regarding the expectation. At the conclusion of the practice session, the investigator asked participants to indicate if they understood the instructions. If a participant was unclear about the instructions, the investigator repeated them for the participant to ensure the participant understood the instructions and expectations prior to completing the three trials. This practice session was not scored. First utilizing this practice session reduced the risk of the first trial being skewed due to the participants being unsure of the instructions. The threat was also controlled by utilizing separate testing materials for the practice session and each of the three trials. Because participants were selected from the same community, interaction of treatment and setting were a potential threat. Consequently, the extent to which the results can be generalized beyond a similar population and sample may be limited.

\section{Strengths and Weaknesses of Design}

A strength of repeated measures design is that the investigator was able to control for the potential influence of individual differences because the study was conducted in one session. Because the total estimated time for a participant to complete the study was 30 minutes to 45 minutes, characteristics of the participants, such as change in age or condition, were not factors. Therefore, differences observed in the results are more likely attributed to treatment conditions (change in lighting) rather than variability in the participant. Further, because the study required participants to attend only one session, attrition was not a factor in the study. The repeated measures design was appropriate for this dissertation study because with each trial, it was assumed the participant's status would revert back to baseline, and there would be no cumulative effects after each 
intervention because different testing materials were used for each trial. For example, in each trial, the participant was asked to read a label on a prescription bottle. While the size of the bottle and font size on the label were the same for each bottle, the words the participant was asked to read on each label were comparable, but different. Lacking a control group, the study may not be considered a true experimental study. However, the investigator helped control for this weakness by utilizing random assignment.

Participants were randomly assigned to a group and the order of lighting intervention was different for each group (Portney \& Watkins, 2009). The dissertation study was considered quasi-experimental.

\section{Participants}

The investigator received administrative approval for participants to be recruited from a retirement community in a large metropolitan area in Arkansas. Residents of the community were 66 years of age or older, and the investigator recruited participants among the 215 residents (193 mailboxes) who resided independently in cottages and apartments within the community.

\section{Sample Size}

Utilizing $\mathrm{G}^{*}$ Power 3 , the investigator estimated 30 participants would be needed for the study, and 30 participants completed the study. For a two-tailed repeated measures analysis for the study with three repetitions, an experiment-wise alpha level of .05 , a moderate Cohens $f$ effect size of 0.25 , an assumed correlation among the repeated measures of 0.50 , and a nonsphericity correction epsilon of 1 , a total sample size of 30 for the group for each of the three lighting levels would yield an actual power of 0.84 (Faul, 2014). 


\section{Inclusion Criteria}

Inclusion criteria included English speaking adults 65 years of age or older. Each participant scored a 12/16 or above on the MMSE-2 BV (PAR, Inc., 2012). Further, participants demonstrated a score of 75 or above on the Colenbrander Mixed Contrast Card Set (Precision Vision, n.d.), defined as minimal vision loss (visual acuity of 20/63), or better with corrective lenses if worn in place when visual acuity was tested.

Participants had to be able to read at an eighth-grade level as evidenced by the ability to read the consent form and the practice testing materials. Participants had to be able to access the testing room within the facility.

\section{Exclusion Criteria}

Exclusion criteria included those who were less than 65 years of age and did not speak English. Also excluded were those who were unable to read at an eighth-grade level or above, and those who did not meet the inclusion criteria of a 12/16 or above on the MMSE-2 BV (PAR, Inc., 2012) and a score of 75 or above, defined as minimal vision loss (visual acuity of 20/63) on the Colenbrander Mixed Contrast Card Set (Precision Vision, n.d.) or better with corrective lenses if worn in place when visual acuity was tested.

\section{Characteristics}

The 30 participants consisted of 24 females and 6 males with a mean age of 83 years $($ range $=66-93$ years $)$. Most were widowed $(n=20)$, and most reported living alone $(n=24)$. Participants had resided within the retirement community for an average of 7 years (range $=0.5-29$ years; one participant did not provide this information). Additional participant information is provided in Chapter 4. 


\section{Recruitment Procedures}

The investigator recruited participants among the independent living residents of the retirement community with a goal of reaching 30 participants for the study. The investigator placed informational flyers in 193 resident mailboxes and throughout the Commons Building. The flyer (see Appendix B) invited residents to an informational meeting held in the Commons Building on December 18, 2014 at which time they were invited to participate in the study. During the meeting, participants were given the opportunity to sign up to participate in the study (see Appendix C), and 37 residents signed up to participate. The investigator and residents communicated via telephone to reach a mutually agreed upon time for participants to complete the study.

\section{Ethical Considerations and Review}

The researcher obtained approval from the IRB of NSU to conduct the study. Once approved by the NSU IRB, the investigator shared this approval and a copy of the proposal with the retirement community facility's Research Oversight Committee for their approval, and the study was completed.

The investigator adhered to the Health Insurance Portability and Accountability Act (HIPAA). Participant information was coded to ensure privacy, and the participants' identities were known only to the investigator. All records were maintained by the investigator in a secured, locked cabinet.

\section{Study Setting}

The dissertation study was conducted in the Commons Building within a retirement community in a large metropolitan area in Arkansas. The retirement community is owned and operated by a large not-for-profit health care system. An office 
in the Commons Building in the retirement community served as the testing center. The testing room was $11 \mathrm{ft} 5$ in. by $7 \mathrm{ft} 6$ in. and contained a desk that was $5 \mathrm{ft}$ by $4 \mathrm{ft} 6$ in. The ambient lighting in the testing room was provided by two ceiling mounted overhead fixtures. Each fixture consisted of four Phillips F32 T8/TL741 700 series, 32-watt, Alto collection G6 fluorescent tubes. The fixtures were controlled by a single on-off light switch located on the wall to the left of the doorway. The total ambient lighting averaged $103 \mathrm{fc}$. Additional lighting was provided and adjusted throughout the procedures utilizing the following lamps:

- A Hampton Bay floor lamp with a reading lamp, which was 71.5 inches tall and contained two bulbs: a 100-watt equivalent with shade, which directed toward the ceiling and a 60-watt equivalent lamp with shade, which directed downward.

- A Mainstays Swivel Organizer desk lamp, which was approximately 10 inches from base to light source and utilized a 60-watt equivalent lamp with a shade, which directed toward the task.

Lamps (bulbs) utilized in the floor lamp and task lamp were as follows:

- EcoSmart 100-watt equivalent bright white CFL, 1,600 lumens, 3,500 K.

- EcoSmart 60-watt equivalent bright white CFL, 800 lumens, 3,500 K.

Desiring to utilize lighting that can be easily achieved in a home setting, the investigator chose lamps that were readily available at local retail stores. The linear fluorescent bulbs already present in the room provided a good source of indirect light, which minimized shadows and glare (Rizzo, 2007). Compact fluorescent bulbs were chosen because they were designed to replace incandescent bulbs and, consequently, are 
becoming much more popular for household use (Rizzo, 2007; Washington State Department of Ecology, n.d.). Further, the bright white bulbs had a color temperature of 3,500 K and were chosen because they provided a good balance between warm color, which many people prefer, and cool color, which is closer to daylight and is good at revealing certain colors (IESNA, 2009; Washington State Department of Ecology, n.d.). Further, the bright white bulbs were chosen because they provided lighting that meets or exceeds recommendations of the IESNA for older adults (IESNA, 2010).

After recruitment and sign-up, upon arrival at the study site, participants were provided a written informed consent statement explaining the study procedures, including potential risks and their right to refuse and/or withdraw at any time. Participants were instructed to wear corrective lenses, if applicable, and if desired.

\section{Instruments and Measures}

\section{Lighting}

Lighting was measured utilizing an Extech Foot Candle Light Meter, Model 401027 (Extech Instruments, 2013). Ambient and task lighting were measured on the desktop at the level of the task. The light meter measured from 0 to 1,999 foot candles of light. The battery-operated device was used by placing the instrument on the desk top with the white lens facing the light source to be measured.

The Informed Consent, Demographic Data, and Practice Trial were conducted under ambient lighting in the testing room $(M=103 \mathrm{fc})$. During each of the three trials, lighting was adjusted according to the Test Lighting Levels. Mean light levels for each light level were as follows:

- Ambient lighting $(M=103 \mathrm{fc}, S D=4.9)$. 
- Ambient lighting with floor lamp $(M=127 \mathrm{fc}, S D=3.3)$.

- Ambient lighting with floor lamp and task lamp $(M=397, S D=5.3)$.

\section{Cognitive Status}

The investigator chose to perform a cognitive screen on participants to ensure they were generally oriented and could follow basic directions as were required during the study. Cognitive status was measured utilizing the MMSE-2 BV (PAR, Inc., 2012), which is designed to provide a quick screen for cognitive impairment in adults 18 to 100 years of age. The MMSE-2 BV (PAR, Inc., 2012) measures registration/recall, orientation to time, and orientation to place. The investigator chose this exam because it is widely used and recognized, and it could be administered in approximately 5 minutes, providing a quick cognitive screen of adults who have not been referred due to cognitive impairment. Reliability and normal range of scores were established utilizing a normative sample of over 1,500 individuals, and validity was established utilizing a sample of patients with Alzheimer's disease and patients with subcortical dementia. Internal consistency coefficients ranged from 0.66 to 0.79 , and interrater reliability coefficients ranged from 0.94 to 0.99 (PAR, Inc., 2012).

\section{Vision}

Visual acuity and contrast sensitivity were measured utilizing The Colenbrander Mixed Contrast Card Set (Precision Vision, n.d.). The card measures high contrast on the left side of the card and low contrast on the right side of the card and is calibrated for 40 $\mathrm{cm}$. The hand-held card contains a cord that ensures viewing distance is accurately maintained as a distance of $40 \mathrm{~cm}$. (16 inches) must be maintained for the measurements to remain valid. One side of the card measures reading acuity, and the other side 
measures letter acuity. The letter acuity side of the card was used in the dissertation study. Scoring measures along the margins of the card indicate standard visual acuity measurements (i.e., 20/20) as well as a Visual Acuity Score (VAS) for each line on the card. For healthy adults, the difference in high contrast versus low contrast lines is usually 1 to 3 . An advantage of the test is that it measures visual acuity and contrast sensitivity in one instrument, and contrast sensitivity is an important component of functional vision. The instrument is designed as a quick screening tool, and the authors recommend additional testing in individuals who demonstrate significant deficits (Colenbrander, n.d.a.; Colenbrander, n.d.b; Precision Vision, n.d.; Precision Vision, 2013).

\section{Effort}

Effort was measured utilizing the RSME (Zijlstra, 1993). The RSME is a unidimensional scale that asks participants to indicate the amount of effort invested into a task by placing a mark along the scale. The RSME is a vertical axis scale ranging from 0 to $150 \mathrm{~mm}$ with every $10 \mathrm{~mm}$ indicated. At various points along the line, at certain anchor points, there are nine descriptive indicators ranging from 3 (not effortful) to 114 (awfully effortful). Participants were asked to mark a point on the scale that was indicative of their perceived effort exerted during performance of the occupational tasks under each lighting level (de Waard, 1996; Wilson et al., 2007). Assessment of the reliability of the RSME indicated reliability of the scale in laboratory $(r=0.88)$ and reallife settings $(r=0.78)$ is acceptable. Additionally, the scale was found to correlate strongly with psychophysiological indicators of mental effort (Wilson et al., 2007). 


\section{Occupational Tasks}

Participants were asked to complete the following occupational tasks (see

\section{Appendix G):}

- Sorting pills (five round, white pills of four different types; variation in pills consisted of size and presence of imprints; total of 20 pills; sets contained different brands/types of pills but each set was consistent; for example, each set had a larger pill and a very small pill) as presented on a tray lined with black felt.

- Sample instructions. While preparing to place your medications in your weekly pill sorter, the pill bottles are knocked over spilling the pills. When I say go, please sort these pills into four different types of pills, placing each type in the corners of the tray.

- Reading a prescription label (actual label on an average size prescription bottle) as presented on a tray lined with black felt.

- Sample instructions. When I say go, please read the patient name at the top of the label, the name of the drug, and the instructions for taking the medication as stated on the label.

- Sorting dark colored socks (four each of the following colors: black, dark brown, navy, dark gray; pattern and texture of the socks remained constant within each set) as presented on a tray lined with white felt.

○ Sample instructions. In preparation for folding the laundry, your socks need to be sorted. When I say go, please sort these socks into four 
different colors, placing a different color of sock in each corner of the tray.

These occupational tasks were selected based on tasks used in previous studies as well as the investigator's personal experience in working with older adults. Also, the investigator attempted to select tasks that were gender neutral. Two of the tasks pertained to medication, a common occupational task of concern for older adults (Beckman, Parker, \& Thorslund, 2005; Skomrock \& Richardson, 2010; Windham et al., 2005). The pill-sorting task in this study was modeled after a procedure utilized by Evans et al. (2010) and Owsley et al. (2001) asked participants to read a prescription label. The sorting sock task was modeled after one utilized by Owsley et al. (2001) who requested that participants pick a designated color of sock from a laundry basket. In the dissertation study, participants were timed as they sorted dark socks of varying colors. The investigator chose to utilize this method to keep all tasks similar and avoid a dichotomous right/wrong answer for this particular task. Further, the occupational tasks were performed against backgrounds that provided high contrast. The white pills were sorted and the white prescription labels read against a black background, and the dark socks were sorted against a white background.

\section{Data Collection Procedures}

Thirty-seven participants agreed to participate in the study. Six participants later declined to participate or failed to follow through with appointment times. Thirty-one participants took part in the study; however, after partaking in the screening process, one participant did not meet the inclusion criteria for the study. Therefore, 30 participants completed the study, meeting sample size requirement for power. Data was collected 
between January 20, 2015, and March 25, 2015. After each participant had signed the informed consent, the investigator conducted two screening tools, the MMSE-2 BV (PAR, Inc., 2012) and the Colenbrander Mixed Contrast Card Set (Precision Vision, n.d.), and recorded scores on the demographic form (see Appendix D). Participants who met minimum criteria on both screenings were asked to continue in the study and were asked to complete the following information on the demographic form: gender, age, education level, and type of eye disease if any (see Appendix D). Upon completion of the demographic form, participants were asked to complete the different tasks according to protocol. As participants were completing each task, the investigator recorded on the study record (see Appendix E) the time for completion of each task as measured with a stopwatch and the average lighting level in footcandles as measured with the light meter. Upon completion of all three tasks, the investigator summed the total time required to complete them. At the conclusion of the three tasks at each light level, the participants were asked to complete the RSME. The investigator indicated the light level on the RSME. Upon completion of all tasks, the investigator placed each participant's information and results in a separate folder.

\section{Data Analyses}

\section{Management of Data}

A paper file, which includes the informed consent, demographic information sheet, study record, scoring sheets for the MMSE-2 BV (PAR, Inc., 2012), Colenbrander Mixed Contrast Card Set (Precision Vision, n.d.), and RSME (Zijlstra, 1993), were maintained on each participant. The information in the paper files was handwritten by the investigator and participants, and each participant was assigned a number by the 
investigator. The investigator recorded the data on Excel spread sheets before entering the data in SPSS with the assistance of a statistician. Participants were identified in Excel and SPSS by assigned number only. Data recorded in Excel and SPSS did not contain patient identifying information, and patient identity was known only by the investigator. Data, including paper and computer files, were maintained in a locked cabinet in the home office of the investigator. Data stored on the investigator's computer is password protected. Data will be maintained for a minimum of 36 months after completion of the study and will be marked to destroy after that time period.

\section{Data Analyses}

Data was analyzed quantitatively using SPSS statistical software (Green \& Salkind, 2008). Descriptive statistics were used to analyze the data and answer the research questions. Descriptive statistics are statistical procedures that are used to organize, summarize, and describe the data.

Measures of variability provided information regarding the distribution of scores and included the range and standard deviation. Standard deviation measures distance from the mean and indicates how spread out scores are in a distribution. Standard deviation and the mean are the most common values used in describing data. Descriptive statistics were calculated for demographic data (gender, age, and education level) and for each of the test items for the subjects. Also calculated were frequency distributions and percentages (Gravetter \& Wallnau, 2009).

A RMANOVA was utilized for the study and allowed for comparison across treatment conditions for each subject. Each participant, serving as his or her own control, performed the occupational task and rated perceived effort on the RSME scale at 
the three different light levels. The RMANOVA was run for the individual and total times required to complete the three occupational tasks at each light level and for the RSME score at each light level. The standard univariate $F$ test was utilized, and significance was defined as $p<.05$. However, two assumptions were violated to the extent the results were not tenable; therefore, a three-way ANOVA was run and yielded the same result. The complete cells assumption was violated because while each Group was exposed to all three lighting levels, each trial did not include all three light levels. The assumption of homoscedasticity was also violated and will be explained in relation to each occupational task, the total of the tasks, and the RSME. Consequently, as a result of these violations, the research questions were analyzed using descriptive statistics to examine the three variables in the study, group, trial number, and lighting level. Using mean completion time for each of the occupational tasks and the total time required to complete the three tasks, each variable was analyzed for the purpose of determining if there were differences in performance based on lighting level. Additionally, mean scores on the RSME were utilized to analyze each variable to determine if there were a difference in participants' perceived effort based on lighting level (Gravetter \& Wallnau, 2009; Portney \& Watkins, 2009).

\section{Format for Presenting Results}

Results were presented in narrative format as well as using tables produced in Microsoft Office Word. Tables were utilized to illustrate the research design and to depict demographic data that defined the participants. Additionally, tables were used to communicate the mean completion times of each group for the three occupational tasks and the total time required to complete the three occupational tasks as well as the mean 
scores of participants on the RSME. Finally, tables were used to illustrate mean completion times based on lighting level. 


\section{Chapter 4: Results \\ Introduction to Chapter}

The goal of the dissertation study was to determine if lighting affects older adults' performance on selected occupational tasks that are visually challenging, and if lighting affects older adults' perceived effort when performing the selected occupational tasks under different lighting levels. Quantitative methods were used to analyze data collected as participants performed the occupational tasks under each lighting level and rated their perceived level of effort while performing the tasks. This chapter provides the results of the study, including demographic data as well as descriptive statistics, which address the following research questions:

1. What is the impact of lighting on older adults' performance on selected tasks that are visually challenging? The null hypothesis stated that changing light levels would result in no significant changes in older adults' performance of visually challenging tasks as measured by time to complete the tasks. The research hypothesis stated that changing light levels would result in at least one significant change in older adults' performance of visually challenging tasks as measured by time to complete the tasks.

2. What is the impact of lighting on perceived effort of older adults while performing tasks that are visually challenging? The null hypothesis stated that changing light levels would result in no significant changes in perceived effort of older adults while performing visually challenging tasks as measured on a self-report scale. The research hypothesis stated that changing light levels 
would result in at least one significant change in perceived effort while performing visually challenging tasks as measured on a self-report scale.

This chapter begins with a discussion of the demographic data collected for the participants in the study, followed by the results of the three-way ANOVA and the descriptive statistics that address the two research questions. A total of five ANOVAs were conducted: reading a prescription label, sorting pills, sorting socks, total time for the three occupational tasks, and score on the RSME. A section will be devoted to each ANOVA followed by a section for the overall findings. Interpretation and significance of the results will be discussed in Chapter 5 .

\section{Data Analysis Results}

\section{Demographic Data}

Descriptive statistics were used to report and analyze demographic information that was provided by each participant (see Appendix D). Descriptive data is presented for the group of 30 participants and when applicable, demographics of each of the three groups of participants were analyzed as well.

Inclusion criteria for the dissertation study included a score of 12 or above on the MMSE-2 BV (PAR, Inc., 2012) and a score of 75 or above on the Colenbrander Mixed Contrast Card Set (Precision Vision, n.d.). The mean score of the 30 participants on the MMSE-2 BV (PAR, Inc., 2012) was 15 with a range of 13 to 16, and the mean score for the Colenbrander Mixed Contrast Card Set (Precision Vision, n.d.) was 95 with a range of 80 to 105 . The mean and standard deviation of each of the three groups' scores on the MMSE-2 BV (PAR, Inc., 2012) and the Colenbrander Mixed Contrast Card Set (Precision Vision, n.d.) can be seen in Table 2. 
Table 2

MMSE-2 BV and Colenbrander Mixed Contrast Card Group Results

\begin{tabular}{cccccc}
\hline Group & MMSE-2 BV mean & $S D^{a}$ & Colenbrander mean & U.S. equivalent & $S D^{a}$ \\
\hline 1 & 14.7 & .949 & 91.5 & $20 / 32-20.15$ & 6.7 \\
2 & 15.7 & .630 & 96.5 & $20 / 25-20 / 20$ & 5.2 \\
3 & 14.4 & .787 & 95 & $20 / 25$ & 15.1 \\
Total & 15.1 & .944 & 94.5 & $20 / 25$ & 5.9 \\
\hline
\end{tabular}

Note. ${ }^{\text {a }} \mathrm{SD}=$ standard deviation.

Thirty participants met the inclusion criteria and completed the informed consent to participate in the study. Demographic data can be seen in Table 3. Of the 30 participants, $80 \%(n=24)$ were female and $20 \%(n=6)$ were male with a mean age of 83 years (range $=66-93$ years). On average, participants had resided in the retirement community for 7 years (range $=0.50-29$ years, $n=29,1$ participant did not provide data) . Two thirds of participants $(n=20)$ were widowed while $20 \%(n=6)$ were married, and $10 \%(n=3)$ were single, and 3\% $(n=1)$ was divorced. Eighty percent $(n=24)$ lived alone, and $20 \%(n=6)$ lived with someone. Ten percent $(n=3)$ had a $12^{\text {th }}$-grade education, 40\% ( $n=15)$ had at least some college experience, and 50\% $(n=15)$ had education beyond a traditional college degree. 
Table 3

General Demographic Data

\begin{tabular}{lcccc}
\hline Variable & Group 1 & Group 2 & Group 3 & Total \\
\hline $\begin{array}{l}\text { Number of participants } \\
\text { Gender }\end{array}$ & 10 & 13 & 7 & 30 \\
$\quad$ & & & & \\
$\quad$ Male & 9 & 3 & 2 & 6 \\
$\quad$ Female & & & 5 & 24 \\
Age & 84.6 & 81.1 & 84.1 & 83.0 \\
$\quad$ Mean & 4.7 & 5.3 & 9.3 & \\
$\quad$ a $D D$ & & & & \\
Years of residency & 7.1 & 7.0 & 8.1 & 7.0 \\
$\quad$ Average & 5.6 & 5.6 & 10.2 & 6.7 \\
$\quad$ a $S D$ & & & & \\
Marital status & 1 & 4 & 1 & 6 \\
$\quad$ Married & 7 & 9 & 4 & 20 \\
$\quad$ Widowed & 2 & 0 & 1 & 3 \\
$\quad$ Single & 0 & 0 & 1 & 1 \\
$\quad$ Divorced & & & & \\
Living status & & 10 & 5 & 24 \\
$\quad$ Alone & 9 & 3 & 2 & 6 \\
$\quad$ With someone & 1 & & & \\
Education & & 2 & 0 & 3 \\
$\quad$ 12 & & & \\
$\quad$ Some college & 7 & 4 & 1 & 12 \\
Beyond college & 2 & 7 & 6 & 15 \\
\hline
\end{tabular}

Note. ${ }^{\mathrm{a}} \mathrm{SD}=$ standard deviation.

When asked about the presence of eye disease, nearly two thirds of participants ( $n$ $=19)$ reported having cataracts while $3 \%(n=1)$ reported having both cataracts and glaucoma. Of the two thirds reporting to have cataracts, $20 \%(n=6)$ also denied having eye disease, yet only $3 \%(n=1)$ indicated having had cataract surgery. Three percent $(n$ =1) indicated having cataracts and having had cataract surgery. Macular degeneration and astigmatism were each reported by $3 \%(n=1)$. Forty-seven percent $(n=14)$ denied 
having eye disease; however, of those, $20 \%(n=6)$ also reported having cataracts.

Specific data regarding reported eye disease can be seen in Table 4.

Table 4

Eye Disease

\begin{tabular}{lcccc}
\hline Disease & Total & Group 1 & Group 2 & Group 3 \\
\hline Cataracts & 18 & 6 & 7 & 6 \\
Macular degeneration & 1 & 1 & 0 & 0 \\
Cataract and macular degeneration & 1 & 0 & 1 & 0 \\
Cataract and glaucoma & 1 & 1 & 0 & 0 \\
Astigmatism & 1 & 0 & 0 & 0 \\
No eye disease & $14^{*}$ & 6 & 6 & 3 \\
\hline
\end{tabular}

Note. $*$ Of these, six participants also reported having a cataract.

Participants were asked about the use of an assistive device, and $87 \%(\mathrm{n}=26)$

denied using one. However, $7 \%(n=2)$ reported using a cane, and 7\% $(n=2)$ reported using a walker and a cane. Two participants did not answer the question but were not using an assistive device when they reported for the study. When asked, $80 \%(n=24)$ denied having fallen in the past year while $20 \%(n=6)$ admitted they had fallen at least once in the past year. When asked the reason for their falls, $7 \%(n=2)$ indicated carelessness while 3\% $(n=1)$ indicated tripping and dizziness. Other reasons for falls were reported as low blood pressure $(3 \%, n=1)$, jumping $(3 \%, n=1)$, and unknown $(3 \%, n=1)$. Specific data regarding assistive device use is in Table 5, and data regarding reported falls is in Table 6. 
Table 5

Assistive Device Use

\begin{tabular}{lcccc}
\hline Assistive device use & Total & Group 1 & Group 2 & Group 3 \\
\hline Walker & 0 & 0 & 0 & 0 \\
Cane & 2 & 0 & 2 & 0 \\
Walker and cane & 20 & 1 & 0 & 1 \\
None & 26 & $9^{*}$ & $11^{*}$ & 6 \\
\hline
\end{tabular}

Note. *One participant did not answer but was not using an assistive device.

Table 6

Falls and Reasons for Falls

\begin{tabular}{|c|c|c|c|c|}
\hline Variable & Total & Group 1 & Group 2 & Group 3 \\
\hline \multicolumn{5}{|l|}{ Falls } \\
\hline Yes & 6 & 2 & 1 & 3 \\
\hline No & 24 & 8 & 12 & 4 \\
\hline \multicolumn{5}{|l|}{ Reasons for falls } \\
\hline Trip & 1 & 1 & 0 & 0 \\
\hline Dizziness & 1 & 0 & 0 & 1 \\
\hline Careless & 2 & 1 & 0 & 1 \\
\hline Other & 3 & 1 & 1 & 1 \\
\hline
\end{tabular}

The demographic questionnaire also asked participants to indicate activities with which they have difficulty. Sixty-three percent $(n=19)$ denied having difficulty with any activities while 37\% $(n=11)$ indicated having difficulty with one or more activities. Seventeen percent $(n=5)$ indicated reading was difficult while $13 \%(n=4)$ reported difficulty locating a number in a telephone directory. Seven percent $(n=2)$ reported difficulty with grooming while $3 \%(n=1)$ reported difficulty with paying bills, writing a check, preparing food, participating in leisure activities, doing laundry, and taking medications. Other activities reported as difficult were golf, memory, and vacuuming (each $3 \%, n=1$, see Table 7). 
Table 7

Activities that are Difficult

\begin{tabular}{lcccc}
\hline Activity & Total & Group 1 & Group 2 & Group 3 \\
\hline Reading & 5 & 3 & 2 & 0 \\
Phone book & 4 & 2 & 2 & 0 \\
Paying bills & 1 & 0 & 1 & 0 \\
Writing checks & 1 & 0 & 1 & 0 \\
Preparing food & 1 & 0 & 1 & 0 \\
Grooming & 2 & 1 & 1 & 0 \\
Leisure & 1 & 1 & 0 & 0 \\
Laundry & 1 & 1 & 0 & 0 \\
Medications & 1 & 1 & 0 & 0 \\
Other & 3 & 0 & 2 & 1 \\
\hline
\end{tabular}

\section{Overview}

Participants completed the three occupational tasks a total of four times: a practice trial followed by Trials 1,2, and 3. The practice set and the three trial sets contained items that were similar yet different. For example, for the sock-sorting task, each trial kit contained a black, dark gray, dark brown, and navy sock, and all the socks in one particular trial set were the same brand and style (see Appendix G). The trial session, which consisted of completing the three occupational tasks under ambient light $(M=103$ fc; $S D=4.9$ ), was completed first along with the RSME followed by completion of Trials 1, 2 and 3 under each of the three lighting levels in varying order, depending on group selection as indicated on the card drawn by the participant. Upon completion of the three occupational tasks in each trial, participants were asked to rate their perceived level of effort for each lighting level using the RSME.

There were three variables (factors) analyzed in the dissertation study: group, trial number, and lighting level. The first factor, group, had three levels and represented the order in which the lighting levels were presented to participants. Participants were randomly assigned to a group by drawing a card. Each of the three cards indicated to the 
investigator a different order in which the lighting levels were presented (see Appendix F). The lighting order was not shared with participants. The second factor was trial number and was indicative of whether it was the first, second, or third time the participant completed the three occupational tasks. The third factor was lighting level and represents the lighting level utilized in the trials (see Table 8).

Table 8

Groups, Trials, and Lighting Levels

\begin{tabular}{ccccc}
\hline & \multicolumn{3}{c}{ Lighting level } & \\
\cline { 2 - 4 } Group & Trial 1 & Trial 2 & Trial 3 & $N$ \\
\hline 1 & Low & Medium & High & 10 \\
2 & High & Medium & Low & 13 \\
3 & Medium & High & Low & 7 \\
\hline
\end{tabular}

A three-way ANOVA (group x trial x light level) was calculated for the time required to complete each of the three tasks (reading a prescription label, sorting pills, and sorting socks) and the total time to complete all three tasks in order to address the first research question regarding the impact of lighting on performance of visually challenging tasks. However, the $F$ statistic could not be calculated for trial number and light level due to violation of the assumption of complete cells, but the $F$ statistic was calculated for group. Therefore, descriptive statistics were utilized to address the research question by examining performance of each the three groups of participants on each of the three tasks as well as the total time required to complete the three tasks.

A three-way ANOVA (group x trial x light level) was also calculated for the RSME in order to address the second research question regarding the impact of lighting on perceived effort of older adults while performing visually challenging tasks.

However, the $F$ statistic could not be calculated for trial number and light level due to violation of the assumption of complete cells, but the $F$ statistic was calculated for group. 
Therefore, descriptive statistics were utilized to address the second research question by evaluating the RSME scores of each the three groups of participants.

In the subsequent sections of the chapter, each occupational task is addressed as follows: the ANOVA; descriptive statistics, including a comparison across groups and the mean lighting level for each occupational task; the total of the three occupational tasks; and the mean score on the RSME. Finally, the research questions were addressed by comparing the three groups across the three occupational tasks and the RSME.

\section{Occupational tasks.}

Reading a prescription label. For the task of reading a prescription label, there was a significant main effect for group: $F(2,81)=4.364, p=.016(p<.05)$. However, Levene's Test of Equality of Error Variances was $p=.000(p<.05)$, suggesting that variances were not equal. Pairwise comparisons of the three groups suggested differences in performance between Group 1 and Group $2(M=3.832, p=.00, p<.05)$ and between Group 1 and Group $3(M=4.068, p=.00, p<.05)$ for this occupational task.

In general, Group 1 required longer to read the prescription labels $(M=13.03, S D$ $=5.18)$ than Group $2(M=9.19, S D=1.98)$ and Group $3(M=8.96, S D=2.64)$. Regardless of the order of the lighting level, for each of the three groups, reading a prescription label took the longest amount of time to complete under medium lighting level (Group 1: $M=14.11, S D=6.83$; Group 2: $M=10.04, S D=2.04$; Group 3: $M=$ 11.24, $S D=2.62$ ). During Trial 1, in which each lighting level was represented, Group 1 took longer to read the prescription label under the low lighting level $(M=13.96, S D=$ 4.19) than Group 2 under the high lighting level $(M=9.81, S D=1.76)$ and Group 3 
under the medium lighting level $(M=11.24, S D=2.62)$. During Trial 2 , Group $1(M=$ $14.11, S D=6.83)$ and Group $2(M=10.04, S D=2.04)$ each took longer to read the prescription label using the medium lighting level than Group $3(M=8.76, S D=1.51)$ did under the high lighting level. During Trial 3, under the high lighting level, Group 1 $(M=11.01, S D=3.95)$ took longer to read the prescription label than Group $2(M=7.73$, $S D=1.28)$ and Group $3(M=6.88, S D=1.73)$ did under the low lighting levels (but Group 1 performed faster under the high lighting level in Trial $3[M=11.01, S D=3.95]$ than they did under low $[M=13.96, S D=4.19]$ and medium lighting levels $[M=14.11$, $S D=6.83$ ] in Trials 1 and 2). Each group read the prescription label in the least amount of time during Trial 3. For Group 1, this trial was the high lighting level, and for Groups 2 and 3, this trial was the low lighting level (see Table 9). When comparing overall means at each lighting level, reading a prescription label required the least amount of time under the low lighting level $(M=9.52)$ as compared to the medium lighting level $(M$ $=11.80)$ and high lighting level $(M=9.86$, see Table 14).

Table 9

Reading Prescription Label

\begin{tabular}{cccccc}
\hline Group & & Trial 1 & Trial 2 & Trial 3 & \\
\hline 1 & & Low & Medium & High & Total \\
& Mean & 13.96 & 14.11 & 11.01 & 13.03 \\
& $S D$ & 4.19 & 6.83 & 3.95 & 5.18 \\
2 & & High & Medium & Low & Total \\
& Mean & 9.81 & 10.04 & 7.73 & 9.19 \\
& $S D$ & 1.76 & 2.04 & 1.28 & 1.98 \\
3 & & Medium & High & Low & Total \\
& Mean & 11.24 & 8.76 & 6.88 & 8.96 \\
& $S D$ & 2.62 & 1.51 & 1.73 & 2.64 \\
\hline
\end{tabular}

Note. ${ }^{\text {a }} S D=$ standard deviation. 
Sorting pills. For the task of sorting pills, there was not a significant main effect for group: $F(2,81)=1.269, p=.287(p$ is not $<.05)$. Levene's Test of Equality of Error Variances was $p=.000(p<.05)$, which suggested that variances are not equal. Because there was not a significant main effect for group, pairwise comparisons could not be made with confidence. Therefore, the descriptive statistics were analyzed in order to address the research question.

Of the three groups, Group 1 took longer to sort the pills in two of the three trials: Trial $2(M=61.85, S D=65.42)$ under the medium lighting level and Trial $3(M=29.67$, $S D=10.85$ ) under the high lighting level while Group 3 took the longest to complete the task in Trial $1(M=106.40, S D=124.86)$ under the medium lighting level. In Trial 1 , Group $3(M=106.40, S D=124.86)$ required longer to sort the pills under the medium lighting level than Group $1(M=71.82, S D=53.35)$ did under the low lighting level and Group $2(M=52.60, S D=24.93)$ did under the high lighting level. Each group required less time to complete each subsequent Trial (Trial $1>$ Trial $2>$ Trial 3) regardless of the lighting level (see Table 10). When comparing the overall means at each lighting level, sorting pills required the least amount of time under the high lighting level $(M=39.62)$ as compared to the medium lighting level $(M=66.48)$ and the low lighting level $(M=$ 39.75, see Table 14). 
Table 10

Sorting Pills

\begin{tabular}{cccccc}
\hline Group & & Trial 1 & Trial 2 & Trial 3 & \\
\hline 1 & & Low & Medium & High & Total \\
& Mean & 71.83 & 61.85 & 29.67 & 54.45 \\
& ${ }^{a} S D$ & 53.35 & 65.42 & 10.85 & 50.82 \\
2 & & High & Medium & Low & Total \\
& Mean & 52.60 & 31.18 & 20.87 & 34.89 \\
& ${ }^{\text {a }} S D$ & 24.93 & 15.24 & 9.07 & 21.80 \\
3 & & Medium & High & Low & Total \\
& Mean & 106.40 & 36.58 & 26.56 & 56.51 \\
& ${ }^{2} S D$ & 124.86 & 22.32 & 15.27 & 78.87 \\
\hline
\end{tabular}

Note. ${ }^{\text {a }} S D=$ standard deviation.

Sorting socks. For the task of sorting socks, there was not a significant main effect for group: $F(2,81)=1.965, p=0.147(p$ is not $<.05)$. Levene's Test of Equality of Error Variances was $p=0.177$. The $p$ value was less than .05 , suggesting that variances are equal. However, because there was not a significant main effect for group, pairwise comparisons could not be made with confidence. Therefore, the descriptive statistics were analyzed in order to address the research question.

In general, Group 1 required longer to sort the socks $(M=24.76, S D=11.86)$ than Group $2(M=18.01, S D=7.58)$ and Group $3(M=20.23, S D=7.13)$. Regardless of the order of the lighting level, for each of the three groups, sorting the socks took the longest amount of time to complete under low lighting level (Group 1: $M=26.78, S D=$ 10.76; Group 2: $M=19.82, S D=7.56$; Group 3: $M=23.40, S D=5.42)$. Group $1(M=$ 21.94, $S D=8.29)$ and Group $2(\mathrm{M}=15.07, \mathrm{SD}=3.98)$ required the least amount of time under the medium lighting level, whereas Group 3 required the least amount of time to sort the socks under the high lighting level (see Table 11). When comparing the overall means at each lighting level, sorting socks required the least amount of time at the 
medium lighting level $(M=19.71)$ as compared to the low lighting level $(M=23.33)$ and the high lighting level $(M=19.96$; see Table 14$)$.

Table 11

Sorting Socks

\begin{tabular}{|c|c|c|c|c|c|}
\hline Group & & Trial 1 & Trial 2 & Trial 3 & \\
\hline \multirow[t]{3}{*}{1} & & Low & Medium & High & Total \\
\hline & Mean & 26.78 & 21.94 & 25.57 & 24.76 \\
\hline & ${ }^{\mathrm{a}} \mathrm{SD}$ & 10.76 & 8.29 & 15.95 & 11.86 \\
\hline \multirow[t]{3}{*}{2} & & High & Medium & Low & Total \\
\hline & Mean & 19.15 & 15.07 & 19.82 & 18.01 \\
\hline & ${ }^{\mathrm{a}} \mathrm{SD}$ & 9.74 & 3.98 & 7.56 & 7.48 \\
\hline \multirow[t]{3}{*}{3} & & Medium & High & Low & Total \\
\hline & Mean & 22.12 & 15.17 & 23.4 & 20.23 \\
\hline & ${ }^{\mathrm{a}} \mathrm{SD}$ & 8.53 & 4.61 & 5.42 & 7.13 \\
\hline
\end{tabular}

Note. ${ }^{\text {a }} \mathrm{SD}=$ standard deviation.

Total of three tasks. For the total of the three tasks, there was not a significant main effect for group: $F(2,81)=1.925, p=0.152(p$ is not $<.05)$. Levene's Test of Equality of Error Variances was $p=.000$. The $p$ value was less than .05 , suggesting that variances were not equal. Because there was not a significant main effect for group, pairwise comparisons could not be made with confidence. Therefore, the descriptive statistics were analyzed in order to evaluate the research question.

Of the three groups, Group 1 required the longest total time to complete the three occupational tasks $(M=92.24, S D=59.66)$, and Group 2 required the least total time $(M$ $=62.09, S D=25.02$ ). However, in Trial 1 in which each lighting level is represented, Group $3(M=139.75, S D=123.03)$ required longer to complete the three tasks under the medium lighting level than Group $1(M=112.57, S D=60.91)$ did under the low lighting level or Group $2(M=81.56, S D=29.34)$ under the high lighting level. Group $1(M=$ 
$66.24, S D=24.76$ ) required longer to complete the three tasks in Trial 3 under the high lighting level than Groups $2(M=48.42, S D=15.58)$ and $3(M=56.83, S D=18.07)$ under the low lighting level. Each group required less time to complete each subsequent Trial (Trial $1>$ Trial $2>$ Trial 3) regardless of light levels (see Table 12). When comparing the overall means at each lighting level, the three tasks required the least amount of time at the high lighting level $(M=69.44)$ as compared to the medium lighting level $(M=97.98)$ and the low lighting level $(M=72.60$, see Table 14$)$.

Table 12

Total of Three Tasks

\begin{tabular}{cccccc}
\hline Group & & Trial 1 & Trial 2 & Trial 3 & \\
\hline 1 & & Low & Medium & High & Total \\
& Mean & 112.57 & 97.90 & 66.24 & 92.24 \\
& a SD & 60.91 & 76.81 & 24.76 & 59.66 \\
2 & & High & Medium & Low & Total \\
& & & 56.3 & 48.42 & 62.09 \\
& Mean & 81.56 & 15.14 & 15.58 & 25.02 \\
3 & a SD & 29.34 & High & Low & Total \\
& & Medium & 60.51 & 56.83 & 85.70 \\
& Mean & 139.75 & 21.26 & 18.18 & 82.25 \\
\hline
\end{tabular}

Note. ${ }^{\text {a }} \mathrm{SD}=$ standard deviation.

Rating scale mental effort. A three-way ANOVA (group x trial x light level) was calculated for the RSME in order to address the second research question regarding the impact of lighting on perceived effort of older adults while performing visually challenging tasks. However, with the ANOVA, the $F$ statistic could not be calculated for trial number and light level due to violation of the assumptions of complete cells, but the $F$ statistic was calculated for group. 
For the RSME, there was not a significant main effect for group: $F(2,81)=.741$, $p=0.480(p$ is not $<.05)$. Levene's Test of Equality of Error Variances was $p=.124(p$ $>.05$ ), which suggested that variances were equal. Because there was not a significant main effect for group, pairwise comparisons could not be made with confidence. Therefore, descriptive statistics were utilized to address the research question by evaluating the RSME scores of each the three groups of participants.

Group 1 rated the most effort on the RSME in each of the three trials as well as the total of the three trials $(M=16.70, S D=15.10)$ as compared to Group $2(M=11.46$, $S D=10.33)$ and Group $3(M=14.52, S D=12.24)$. In Trial 1 in which each lighting level is represented, Group 1 rated the most effort. Group 1 and Group 3 rated less effort with each subsequent trial (Trial $3<$ Trial $2<$ Trial 1). While Group 2 rated the most effort in Trial 1 under the high lighting level $(M=14.00, S D=12.49)$, it rated less effort in Trial 2 under the medium lighting level $(M=9.69, S D=8.78)$ than it did in Trial 3 under the low lighting level $(M=10.69, S D=9.70$, see Table 13). When comparing the overall mean at each light level, completing the tasks under the high light level required the least amount of effort $(M=12.95)$ as compared with the medium light level $(M=$ 15.31) and the low light level $(M=14.42$, see Table 14$)$. 
Table 13

Rating Scale Mental Effort

\begin{tabular}{|c|c|c|c|c|c|}
\hline Group & & Trial 1 & Trial 2 & Trial 3 & \\
\hline \multirow[t]{2}{*}{1} & & Low & Medium & High & Total \\
\hline & $\begin{array}{c}\text { Mean } \\
\text { aSD }\end{array}$ & $\begin{array}{l}21.00 \\
18.27\end{array}$ & $\begin{array}{l}16.10 \\
14.93\end{array}$ & $\begin{array}{l}13.00 \\
11.92\end{array}$ & $\begin{array}{l}16.70 \\
15.10\end{array}$ \\
\hline \multirow[t]{2}{*}{2} & & High & Medium & Low & Total \\
\hline & $\begin{array}{l}\text { Mean } \\
{ }^{a} S D\end{array}$ & $\begin{array}{l}14.00 \\
12.49\end{array}$ & $\begin{array}{l}9.69 \\
8.78\end{array}$ & $\begin{array}{c}10.69 \\
9.70\end{array}$ & $\begin{array}{l}11.46 \\
10.33\end{array}$ \\
\hline \multirow[t]{2}{*}{3} & & Medium & High & Low & Total \\
\hline & $\begin{array}{c}\text { Mean } \\
{ }^{a} S D\end{array}$ & $\begin{array}{l}20.14 \\
13.53\end{array}$ & $\begin{array}{l}11.86 \\
10.51\end{array}$ & $\begin{array}{l}11.57 \\
12.27\end{array}$ & $\begin{array}{l}14.52 \\
12.24\end{array}$ \\
\hline
\end{tabular}

Note. ${ }^{\mathrm{a}} \mathrm{SD}=$ standard deviation.

Table 14

Overall Lighting Means Requiring Greatest and Least Time

\begin{tabular}{ll}
\hline Tasks & Mean lighting level requiring greatest time/least time \\
\hline Rx label & Medium/Low \\
Pills & Medium/High \\
Socks & Low/Medium \\
Total & Medium/High \\
& Lighting level requiring greatest/least effort \\
RSME & Medium/High \\
\hline
\end{tabular}

\section{Findings}

It was hypothesized that changing lighting levels would result in no significant changes in older adults' performance of visually challenging tasks as measured by time to complete the tasks and that changing light levels would result in no significant changes in perceived effort of older adults while performing visually challenging tasks as measured on a self-report scale. Due to violation of the complete cells assumption for the ANOVA, the $F$ statistic could not be computed for trial and light level, but it was computed for group. Therefore, the research questions were evaluated utilizing descriptive statistics. The first research question inquired as to the impact of lighting on older adults' 
performance on tasks that are visually challenging. It was evaluated by examining how the groups performed on each task and the total of the three tasks utilizing the mean time required to complete each occupational task and the mean total time required to complete the three tasks under each lighting level. Additionally, the overall mean completion time for each occupational task and the total of the three tasks under each lighting level was examined.

For the task of reading a prescription label, there was a significant main effect between Group 1 and Group $2(M=3.832, p=.00, p<.05)$ and between Group 1 and Group $3(M=4.068, p=.00, p<.05)$. The mean time for Group 1 to complete the task $(M=13.03, S D=5.18)$ exceeded that of Group $2(M=9.19, S D=1.98)$ and Group $3(M$ $=8.96, S D=2.64)$. In Trial 1 in which each lighting level was represented, reading a prescription label required the least amount of time under the high lighting level $(M=$ $9.81, S D=1.76)$ and the longest under the low lighting level $(M=13.96, \mathrm{SD}=4.19) . \quad$ In terms of actual lighting levels, each group required longer to complete the task under the medium lighting level (Group 1: $M=14.11, S D=6.83$; Group 2: $M=10.04, S D=2.04$; Group 3: $M=11.24, S D=2.62$ ) than the low or high lighting levels, regardless of trial number. The overall mean time for reading a prescription label was the least at the low light level $(M=9.52)$ and the greatest at the medium light level $(M=11.80$, see Table 14). Therefore, the descriptive statistics suggested there were differences in performance in the groups under the different lighting levels. While there was not a definitive light level at which participants performed more quickly, participants did consistently require longer to read prescription labels under the medium lighting level. 
For the task of sorting pills, because there was not a main effect, the research question was evaluated utilizing descriptive statistics. In Trial 1 in which each lighting level was represented, sorting pills under the high lighting level $(M=52.60, S D=24.93)$ required the least amount of time while sorting pills under the medium lighting level ( $M$ $=106.40, S D=124.86$ ) required greatest amount of time. However, each group required less time to complete each subsequent trial (Trial $1>$ Trial $2>$ Trial 3 ) regardless of the lighting level. A comparison of overall means for sorting pills at each lighting level also demonstrated that completing the task under high lighting $(M=39.62)$ required the least amount of time, whereas sorting pills under medium lighting required the most time $(M=$ 66.48). Therefore, the descriptive statistics support the research question that questions the impact of lighting on older adults' performance of tasks, which are visually challenging based on time to complete tasks. Although statistical significance could not be determined, the descriptive statistics do support a difference in performance of tasks under different lighting levels. For this occupational task, participants generally completed the task the quickest under the high lighting level and required the most time under the medium lighting level.

For the task of sorting socks, because there was not a main effect, the research question was evaluated utilizing descriptive statistics. In Trial 1 in which each lighting level is represented, sorting socks required the least amount of time under the high lighting level $(M=19.15, S D=9.74)$ and the greatest amount of time under the low lighting level $(M=26.78, S D=10.76)$. For Groups 1 and 2, sorting the socks required the least amount of time under the medium lighting level while Group 3 required the least time under the high lighting level. Each group required the greatest amount of time to 
complete the task under the low lighting level. When comparing the overall means at each lighting level, sorting socks required the least amount of time at the medium lighting level $(M=19.71)$ and required the most time at the low lighting level $(M=23.330)$. Therefore, the descriptive statistics supported the research question that inquired as to the impact of lighting on older adults' performance of tasks, which are visually challenging based on time to complete tasks. Although statistical significance could not be determined, the descriptive statistics do suggest a difference in performance of tasks under different lighting levels. For this occupational task, participants generally completed the task the quickest under the medium lighting level and required the most time under the low lighting level.

For the total of the three tasks, because there was not a main effect, the research question was evaluated utilizing descriptive statistics. During Trial 1, in which each lighting level was represented, completing the three tasks required the least amount of time under the high lighting level and the longest under the medium lighting level. However, each group required less time to complete each subsequent trial (Trial $1>$ Trial $2>$ Trial 3) regardless of the lighting level. When comparing the overall means at each lighting level, the three tasks required the least amount of time at the high lighting level $(M=69.44)$ and required the most at the medium lighting level $(M=97.98)$. Therefore, the descriptive statistics supported the research question that posits the impact of lighting on older adults' performance of tasks, which are visually challenging based on time to complete tasks. Although statistical significance could not be determined, the descriptive statistics do suggest a difference in performance of tasks under different lighting levels. When analyzing the total time required to complete the three occupational tasks, 
participants generally completed the three tasks the quickest under the high lighting level and required the most time to complete under the medium lighting level.

The second hypothesis stated that changing lighting levels would result in no significant changes in perceived effort of older adults while performing visually challenging tasks as measured on a self-report scale, and the research question inquired as to the impact of lighting on perceived effort of older adults while performing tasks that are visually challenging. The research question was evaluated by examining the mean score of each group on the RSME. Because there was not a significant main effect, the research question was evaluated utilizing descriptive statistics. In Trial 1 in which each lighting level was represented, the high lighting level was rated as requiring the least amount of effort and the low lighting level was rated as requiring the most amount of effort. Group 1 rated the most effort on the RSME in each of the three trials as well as the total of the three trials $(M=16.70, S D=15.10)$ as compared to Group $2(M=11.46$, $S D=10.33)$ and Group $3(M=14.52, S D=12.24)$. Groups 1 and 3 rated less effort with each subsequent trial (Trial $3<$ Trial $2<$ Trial 1). However, Group 2 rated the most effort in Trial 1 under the high lighting level $(M=21.00, S D=18.27)$. Yet, they rated less effort in Trial 2 under the medium lighting level $(M=9.69, S D=8.78)$ than they did under the low lighting level $(M=11.57, S D=12.27)$ in Trial 3. When comparing the overall mean at each lighting level, completing the tasks under the high lighting level required the least amount of effort $(M=12.95)$ while completing the tasks under the medium lighting level $(M=15.31)$ required the most effort. While evidence points to a practice effect with Groups 1 and 3 that reported less effort with each subsequent trial, Group 2 rated less effort under the medium lighting level in Trial 2 than it did under the 
low lighting level in Trial 3. Further, in Trial 1 in which each lighting level was represented, the low lighting level (Group 1) was rated as requiring the most effort while the high lighting level (Group 2) was rated as requiring the least effort. Therefore, the descriptive statistics supported the research question that postulates the impact of lighting on older adults' perceived effort while performing tasks, which are visually challenging based on a self-report scale. Although statistical significance could not be determined, the descriptive statistics do suggest a difference in performance of tasks under different lighting levels. Overall, more lighting seemed to equate to less perceived effort and less lighting lead to greater perceived effort.

\section{Summary}

When comparing overall means at each lighting level for each occupational task and the total of the three tasks, performing under medium light level required the greatest amount of time for reading a prescription label, sorting pills, and the total of the three occupational tasks. Sorting socks required the greatest mean time under the low lighting level. The lighting level in which performing the occupational tasks required the least mean time varied and the time difference between lighting levels was often quite small. Reading a prescription label required the least mean time under the low lighting level. However, the difference in mean time between the low lighting level and the high lighting level was 0.34 seconds. Sorting the pills required the least mean time under the high lighting level, but the difference in mean time between high and low lighting levels was 0.13 seconds. Further, sorting socks required the least mean time under the medium lighting level $(M=19.71)$, but the difference in mean time between high $(M=19.96)$ and 
medium lighting levels was 0.25 seconds. The mean time for the total of the three occupational tasks was the least under the high lighting level.

When examining the performance of each occupational task, sorting pills and the total of the three tasks required less time with each subsequent trial (Trial $3<$ Trial $2<$ Trial 1) regardless of group or lighting level. Reading a prescription label required the most time under the medium lighting level regardless of group or order and required the least time during Trial 3 regardless of lighting level. Sorting the socks required the most time under the low lighting level regardless of group and order and required the least amount of time for Groups 1 and 2 under the medium lighting level and for Group 3 under the high lighting level, all of which are in Trial 2 rather than Trial 3. When examining overall means as well as the performance on each occupational task, the descriptive statistics do suggest a difference in performance of tasks under different lighting levels.

For the RSME, Groups 1 and 3 rated less effort with each subsequent trial (Trial 3 $<$ Trial $2<$ Trial 1), and Group 1 consistently rated the most effort over Groups 2 and 3 in each trial. However, while Group 2 rated the most effort in Trial 1 under high lighting, it rated more effort in Trial 3 under the low lighting level than it did in Trial 2 under the medium lighting level. The descriptive statistics suggested a difference in rating of perceived effort under different lighting levels (see Table 15). 
Table 15

Lighting Levels Requiring Greatest and Least Time and Effort

\begin{tabular}{|c|c|c|c|c|c|}
\hline Group & \multicolumn{5}{|c|}{ Tasks and RSME } \\
\hline & $\underline{\mathrm{Rx} \text { label }}$ & $\underline{\text { Pills }}$ & $\underline{\text { Socks }}$ & $\underline{\text { Total }}$ & $\underline{\mathrm{RSME}}$ \\
\hline & \multicolumn{4}{|c|}{ Lighting level requiring greatest time/least time } & $\underline{\text { Greatest/least effort }}$ \\
\hline 1 & Medium/High & Low/High & Low/Medium & Low/High & Low/High \\
\hline 2 & Medium/Low & High/Low & Low/Medium & High/Low & High/Medium \\
\hline 3 & Medium/Low & Medium/Low & Low/High & Medium/Low & Medium/Low \\
\hline
\end{tabular}




\section{Chapter 5: Discussion \\ Introduction to Chapter}

The overall aims of this study were to investigate the effect of lighting on older adults' ability to perform selected occupational tasks that are visually challenging and to determine if lighting affects older adults' perceived effort when performing the selected occupational tasks under different lighting levels. The research questions were the following:

1. What is the impact of lighting on older adults' performance on selected tasks that are visually challenging?

2. What is the impact of lighting on perceived effort of older adults while performing tasks that are visually challenging?

Lighting has been shown to affect performance in individuals with and without visual impairment (Bowers et al., 2001; Davis \& Garza, 2002; Eldred, 1992; Fosse \& Valberg, 2004; Haymes \& Lee, 2006; Izso, Lang, et al., 2009; Petrofsky \& Cuneo, 2008) although many of the studies were conducted utilizing numeric and reading tests (Bowers et al., 2001; Eldred, 1992; Eperjesi et al., 2006; Fosse \& Valberg, 2004; Haymes \& Lee, 2006; Izso, Lang et al., 2009) rather than the functional tasks individuals complete on a daily basis. Studies measuring performance of functional tasks under different lighting conditions are very limited (Charness \& Dijkstra, 1999; Evans et al., 2010). Also, studies on lighting and performance have been conducted by other disciplines, such as architecture, engineering, gerontology, home economics, and psychology, rather than by occupational therapists. Further, studies of lighting in home environments have revealed concerns (Cullinan et al., 1979; Cumming et al., 1999; Gill et al., 1999; Greene 
et al., 2009; Iwarsson \& Isacsson, 1996) because light levels are often below recommended standards (Charness \& Dijkstra, 1999; Lindner et al., 2001; Simpson \& Tarrant, 1983). Nevertheless, rather than introduce additional lighting, older adults tend to rely on existing ambient lighting, describing it as adequate even when it is not (Bakker et al., 2004; Lindner et al., 2001).

Effort, a difficult concept to describe and measure (Yeo \& Neal, 2004), has been studied by few researchers (De Morree \& Marcora, 2010; Fraser et al., 2010; Geruschat \& Turano, 2007; Lewis-Evans et al., 2010). In studying effort, Zijlstra (1993) distinguished between workload, which is external and pertains to the objective complexity of task demands, and effort investment, which relates to an individual's performance potential and accounts for changes in the individual, such as responses to stress, which may affect work capacity. Maintaining that mental workload could best be measured utilizing subjective measures, he developed the RSME as a means of measuring an individual's invested effort when performing a task. Further, he acknowledged the relationship between effort and time, maintaining there is always a trade-off between the two. When pressed for time, individuals may exert more effort in order to complete a task in a timely manner; however, if they feel they have ample time to perform a task, then they may perceive less effort is exerted.

Performance of specific occupational tasks under measured lighting levels has not been studied nor has an individual's perceived effort while performing occupational tasks under different lighting levels. Therefore, this dissertation study examined the impact of lighting on performance of occupational tasks in older adults, and it explored the impact that lighting had on the perceived effort older adults exerted when performing 
occupational tasks requiring near vision under different lighting levels. Investigating performance and perceived effort during performance captured both the objective and subjective aspects of occupational performance (McColl \& Pollock, 2005). The results of the dissertation study were analyzed and interpreted by the time required to complete each occupational task, the total time required to complete the three occupational tasks, and the perceived effort of participants while performing the occupational tasks in order to answer the research questions stated above. This chapter addresses the interpretation of results, implications for occupational therapy practice, future research, limitations, recommendations for occupational therapy practice, and future research.

\section{Interpretation of Results}

Because a violation of the assumption of complete cells prevented accepting or refuting the hypotheses, descriptive statistics were utilized to evaluate the research questions. The research questions evaluated the impact of lighting on older adults' performance of tasks that are visually challenging as well as the impact of lighting on older adults' perceived effort when performing the visually challenging tasks. The analysis of the descriptive data suggested a difference in older adults' performance under different lighting levels and also suggested a difference in their perceived effort while performing the tasks under different lighting levels. While the first research hypothesis that changing light levels will result in at least one significant change in occupational task performance on visually challenging tasks as measured by time to complete tasks cannot be rejected based on the descriptive data, the results certainly affirm the need for further research, which addresses the hypothesis. Further, while the second hypothesis that changing light levels will result in at least one significant change in perceived effort 
while performing visually challenging tasks as measured on a self-report scale also cannot be refuted based on the descriptive data; the results warrant further investigation of this hypothesis as well. The results of the study will be discussed in terms of the three variables (group, trial number, lighting level), the three occupational tasks, the total of the three occupational tasks and the RSME, and the research questions will be addressed utilizing the descriptive data. Because the results of each individual occupational task varied, they will be discussed individually and as a total.

\section{The Variables}

Groups. The groups of participants were fairly equal in terms of scores on the MMSE-2 BV (PAR, Inc., 2012) and the Colenbrander Mixed Contrast Card Set (Precision Vision, n.d.). Group 2 performed on average a point higher on the MMSE-2 $\mathrm{BV}$, and Group 2 participants also appeared to have better vision than participants in Groups 1 and 3. The groups were also fairly equal in terms of other general demographic data; the mean age of Group 2 was about 3 years younger than the mean age of Groups 1 and 3. The groups were fairly equal in terms of reported eye disease with cataract being the most commonly reported disease in all three groups. Two participants in Group 2 and one participant each in Groups 1 and 3 reported using assistive devices for ambulation. While two participants in Group 1, one participant in Group 2, and three participants in Group 3 reported falling within the past year, none of these participants also reported using an assistive device to ambulate. Four participants in Group 1 reported difficulty with one or more activities while five people in Group 2 and one person in Group 3 reported difficulty with one or more activities. 
Because participants were randomly assigned to a group based on their selection of a card, the groups were not matched based on any demographic criteria. The cards indicated to the investigator the order in which the lighting levels would be presented, but this information was not known to the participant. While the size of the groups varied from seven to 13 participants, the groups were fairly equitable in terms of age, inclusion criteria, and reported functional level; participants in Group 1 were slightly older and had poorer vision than participants in Groups 2 and 3, and Group 2 was slightly younger and had higher visual and cognitive scores. For two of the three occupational tasks (reading a prescription label and sorting socks) and the total of the three occupational tasks, Group 1 participants required longer to complete the occupational tasks. They also rated more effort on the RSME than the other two groups. Further, in Trial 1 in which each lighting level was represented and no learning had occurred, Group 1 participants required longer to complete two of the three tasks. These results are important to note because the ANOVA indicated a significant effect between Groups 1 and 2 and Groups 1 and 3 for the task of reading a prescription label. Additionally, participants in Group 2 performed more quickly on two of the three tasks (sorting pills and sorting socks) and the total of the three tasks, and they rated the least amount of effort on the RSME. Despite small differences in average age, scores on the MMSE-2 BV, and scores on the Colenbrander Mixed Contrast Card, the groups were fairly equitable and performed the same occupational tasks. Therefore, it is possible that the differences observed in groups could be attributed to the changes in the lighting levels.

Trials. A practice session preceded the three trials in order to allow participants to ask questions and ensure they understood the instructions prior to the timed trials. The 
investigator provided the practice session in order to eliminate the possibility that timed results in Trial 1 were influenced by participants not understanding the instructions. Despite the use of different but similar sets of materials for each occupational task, participants in each group performed the pill sorting task in less time with each subsequent trial, and the total time for the three trials reflected less time required for each subsequent trial as well. Additionally, participants in Groups 1 and 3 also reported less effort when performing each subsequent trial. Participants were not penalized for inaccuracies when completing the tasks. It is possible that they realized completion time was being monitored but the accuracy of their work was not, and this realization may have contributed to their focusing on finishing as quickly as possible rather than focusing on the accuracy of their work. Also, in an effort to avoid one set of pills being deemed "easier" than another, the investigator designed sets of pills that were very similar in nature. For example, each set contained one pill that was slightly larger than the others. The other pills, which were very close in size, differed in the presence or absence of an imprint and the type of imprint. It is possible that each subsequent trial was completed in less time because participants learned to sort by size and look for the presence or absence of imprints, examining the pills less closely with each subsequent trial. The results from the dissertation study seemed to indicate a practice effect for the pill sorting task when participants completed the occupational task in less time with each subsequent trial.

The total time required by participants to complete the three occupational tasks as a whole was less with each subsequent trial as well. However, this time difference seems to be due to the shorter time results of the pill sorting task and Group 3's quicker performance for reading a prescription label, the only other task that resulted in 
participants requiring less time with each subsequent trial, both of which indicate a possible learning effect. The results of Groups 1 and 2 for the prescription label task and the results of Groups 1, 2, and 3 on the sock-sorting task are not indicative of participants requiring less time with each subsequent trial and consequently do not suggest a practice effect.

Lighting levels. When examining the mean time required to complete the occupational tasks under the different lighting levels, the time varied for each occupational task. There was no clear evidence that all the occupational tasks were completed in less time under a certain lighting level. When examined individually by evaluating mean completion times for each lighting level, each occupational task was completed the most quickly at a different lighting level with each lighting level represented. Sorting pills required the least mean time under the high lighting level while reading a prescription label required the least amount of mean time under the low lighting level, and sorting socks required the least amount of mean time under the medium lighting level. Combining the completion time for each occupational task yielded a total mean time for the three tasks, and as a whole, the three occupational tasks were completed in the least mean time under the high lighting level.

Of the three occupational tasks, sorting pills required the greatest completion time for participants in each of the three groups. The established relationship between time and effort (Zijstlra, 1993) seemed to indicate sorting pills was a tedious task for participants. Further, when observing overall mean time, this occupational task required the least amount of time under the high lighting level $(M=397 \mathrm{fc})$. While the difference in overall mean between the high and low lighting levels was small, nevertheless, the 
results suggested that performance varied under different lighting levels and pointed to higher lighting levels being associated with faster performance when sorting the pills. This finding differs from that of Evans et al. (2010), who had older adults complete a similar pill sorting task under lighting levels of 50 lux, 200 lux, and 800 lux, and found participants performed best under 200 lux (18.6 fc). However, none of the lighting levels in that study met IESNA-recommended lighting standards for older adults performing such a tedious task. IESNA recommends a minimum of $100 \mathrm{fc}$ while reading/studying, $150 \mathrm{fc}$ for kitchen counter activities, and $200 \mathrm{fc}$ for performing hobbies. Each of the lighting levels in the dissertation study met or exceeded these recommended standards (IESNA, 2010).

Reading the prescription label required the least amount of mean time under the low lighting level $(M=103 \mathrm{fc})$. While this lighting level met IESNA recommendations of $100 \mathrm{fc}$ for older adults when reading, the results differed from those of other researchers who studied reading performance in adults with ARMD, utilizing lighting levels ranging from 50 to 7532 lux (4.6 fc-700 fc). Other researchers found participants performed best under higher lighting levels (3,000 lux-7,532 lux), and they agreed that lighting levels should be individualized, and further study is needed to determine optimal lighting levels (Bowers et al., 2001; Eldred, 1992; Haymes \& Lee, 2006). However, caution should be used in comparing the results of the dissertation study with previous studies that utilized participants who had ARMD because only two participants in the dissertation study reported having ARMD while more than half reported having cataracts. In the dissertation study, while the low lighting level provided less lighting on the task than the medium and high lighting levels, the low lighting level did meet IESNA 
standards and appeared to provide adequate lighting for completing the task. Perhaps the medium and high lighting levels created glare that limited participants' performance at those lighting levels, or perhaps the placement of the lamps was perceived as a distraction or hindrance.

Sorting socks required the least amount of mean time under the medium lighting level $(M=127 \mathrm{fc})$. While distinguishing dark colors becomes increasingly difficult as one ages (Sekular \& Sekular, 2000), and older adults have acknowledged difficulty distinguishing clothing colors (Barstow et al., 2011), only one identified study has examined older adults' ability to distinguish dark colored clothing (Owsley et al., 2001), and lighting was not a variable in that study. Therefore, there are no studies with which to compare the results of this dissertation study. Perhaps, the fact that participants in this dissertation study sorted socks in the least amount of mean time under the medium lighting level rather than the high or low lighting levels supports the stance that more light is beneficial to a point, but too much light can be detrimental, possibly creating glare. Nonetheless, because the difference in mean performance of high and medium lighting levels was very small (0.25 seconds), but the mean difference in performance between medium and low lighting levels was nearly 4 seconds, which seemed to indicate for this task that more light was beneficial, thus suggesting that lighting may influence performance. However, the small difference in medium and high light levels could suggest the benefit of increased lighting beyond the medium light level is minimal.

There was more consistency in the lighting level that required the most time for occupational task completion. Reading a prescription label and sorting pills required the most mean time under the medium lighting level as did the total for the three 
occupational tasks. This finding suggests the addition of the floor lamp alone was not beneficial in reading the prescription label or sorting pills nor was it beneficial when considering the total completion time for the three tasks. Furthermore, the addition of the floor lamp and the task lamp was not beneficial when reading the prescription label. It is possible that the presence of the floor lamp was more distracting than beneficial. The lamp, which was 71.5 inches tall, was perhaps too far from the occupational task to be of benefit as the lamp increased the lighting on the task a relatively small amount $(M=24$ fc) above the ambient lighting in the room. Perhaps the floor lamp created a shadow or glare as participants read the prescription labels and sorted pills. However, sorting socks required the most mean time under the low lighting level rather than the medium or high lighting levels, which suggests that some degree of increased lighting was beneficial to participants as they performed that task. Perhaps a consideration for future studies is whether different lighting levels are required depending on the properties of the materials used in the occupational tasks. For example, sorting pastel colored pills may require different lighting needs than sorting dark colored socks.

Many studies of lighting and performance have been conducted in lighting laboratories. However, this investigator wanted to focus on lighting levels that were readily available and achievable in home environments where occupational performance takes place. Therefore, the lighting levels for this dissertation study were chosen based on a review of the literature and standards recommended by IESNA for older adults when performing selected tasks (IESNA, 2010). Despite established standards, much disagreement surrounds which levels are optimal lighting levels for older adults (Christenson, 1990; Kolanowski, 1992; Moller, 2008; Noell-Waggoner, 2004; Watson, 
2001; Young, 2012). Therefore, another consideration when selecting lighting for the dissertation study was choosing lamps that were readily accessible to older adults in the community. The lamps, which were economically priced, were purchased at national retail chain stores. There was little difference in footcandles of the low $(M=103 \mathrm{fc})$ and medium $(M=127 \mathrm{fc})$ light levels, and participants often required the most time to complete tasks under the medium lighting level. To achieve the medium lighting level, the investigator utilized a floor lamp placed based on recommended practice on the right side of the participant (IESNA, 2010). Participants were seated at a desk with each occupational task directly in front of them; however, they were not restrained from leaning forward or back in the chair. Therefore, it is possible the presence of the floor lamp was a distraction, or the light from the floor lamp created a glare or a shadow on the occupational task. It is also possible that the participant's own body cast a shadow on the occupational task. To achieve the high lighting level, in addition to lighting provided by the ambient lighting and floor lamp, a task lamp was placed on the desk in front of the participant. It is possible that the presence of this additional light caused glare or shadows on some of the occupational tasks. Also, the trials were conducted consecutively with only a few minutes in between each one to allow the investigator to adjust the lighting level and testing materials. It is possible that participants would have benefited from more time to adjust to the change in lighting levels. However, realistically, older adults may or may not allow time for adjustment when moving between tasks and lighting levels in the home environment. Also, because light reflects differently off different surfaces (Krusen, 2010), the effects of lighting could have differed for each occupational task based on the background and the nature of the 
materials in each task. For example, light may have reflected differently off dark socks against a white background than it did the white pills against a black background. To allow for sufficient contrast and minimal glare, participants read prescription labels and sorted pills against a black, felt background and sorted dark socks against a white, felt background (IESNA, 2010; Noell-Waggoner, 2004).

To compensate for aging vision, researchers have demonstrated that older adults need more light (Boyce, 2003; Kolanowski, 1992; Krusen, 2010; Moller, 2008; NoellWaggoner, 2004; Perlmutter, 2012; Rizzo, 2007; Young, 2012). The greatest amount of light in this study was $M=397 \mathrm{fc}$, provided by adding a floor lamp and task lamp to the ambient lighting. This level of lighting seemed to benefit participants in Group 1 the most: the group with the greatest mean age and the poorest vision. Rather than simply adding more light, as suggested by the results of the dissertation study, consideration needs to be given not only to the amount of light but also to the type of lamp and the placement of additional lamps. In the dissertation study, the addition of a floor lamp only, which added slightly more lighting, seemed to impede performance as indicated by additional time and effort expended by participants while performing some occupational tasks. Although the floor lamp was placed on the right side of participants based on recommended practice (IESNA, 2010), perhaps its presence was a distraction or cast a shadow or glare upon the task and impeded performance. Although the investigator purposefully selected lamps that are readily available to the older adult population at national chain retailers, the floor lamp provided an additional $M=24 \mathrm{fc}$, a relatively small increase in the overall lighting on the task. Perhaps the presence of the lamp over 
the shoulder of the participant created more of a distraction than a benefit during task performance.

This dissertation study also points to the need for further investigation of the implications of aging and vision. While the physiological changes in vision as one ages are well documented (Rizzo, 2007; Salvi et al., 2006), the effect of aging changes in vision on occupational performance are not as well understood nor is the effect of environmental factors, such as lighting, on occupational performance. This dissertation study points to the need for interventions that focus on lighting in the environment. However, lighting is not an environmental intervention in which one size fits all (American Lighting Association, 2011; Halloin, 1995; Perlmutter, 2012; Young, 2012). As was suggested in this dissertation study, lighting levels that support optimal occupational performance may be specific to the occupational task as well as the older adult's individual needs.

\section{The Occupational Tasks and RSME}

The occupational tasks were chosen because they are visually challenging and because they are tasks with which older adults experience difficulty (Barstow et al., 2011; Brunnstrom et al., 2004; Cook, 2009; Henriques et al., 2012; Lam et al., 2011; MacLaughlin et al., 2005; Owsley et al., 2001; Skomrock \& Richardson, 2010; Windham et al., 2005; Yasein et al., 2013). Normal age-related changes in vision can make occupational tasks requiring near vision difficult and can negatively affect occupational performance (Robnett, 2008). Person factors, such as changes in vision, often cannot be changed and may negatively affect an individual's performance skills. According to the PEO model, when person factors, such as age-related visual changes, interact with the 
occupations an older adult wishes to perform, occupational performance may be compromised. However, when environmental factors, such as lighting, support occupational performance, the demands of an occupational task are lessened thereby enhancing occupational performance. When person factors, the environment, and occupations closely interact, occupational performance is supported; however, when these factors do not closely interact, occupational performance is negatively affected, resulting in diminished occupational performance (Sabata, 2008). Optimal lighting can potentially meet the increased visual needs created by age-related visual changes in older adults and enhance occupational performance. In this dissertation study, the lighting level under which participants performed the quickest varied by task. However, participants generally required the most time and reported the most effort when performing under the medium lighting level, and they reported the least amount of effort when performing under the high lighting level. Both the objective (time) and subjective (effort) aspects of occupational performance are affected by environmental factors (lighting) as suggested by the dissertation results. The results of this dissertation study point to the need for a greater understanding of optimal lighting levels for older adults when performing visually challenging tasks.

The investigator developed four testing sets of similar yet different materials. For example, each set contained a prescription bottle with a label indicating a name, medication, and instructions; however, the exact words varied on each bottle. The investigator designed the testing sets so they were very similar in an effort to avoid completion times being affected by a testing set being perceived as "easier" or "harder." The trial session was utilized to ensure that participants knew the expectations of the 
occupational task, for example, which information to read on the bottle and where the information was located. In so doing, the investigator hoped to avoid extra time on the first trial being attributed to unfamiliarity with the materials or expectations of the occupational task. The occupational tasks chosen by the investigator were supported by the literature (Beckman et al., 2005; Evans et al., 2010; Owsley et al., 2001; Skomrock \& Richardson, 2010; Windham et al., 2005) and seemed appropriately challenging. For example, one of the occupational tasks required participants to read a prescription bottle, and this task seemed an appropriate choice given that on the demographic information sheet, a total of five participants reported difficulty with reading as well as with other tasks that require reading, such as locating a listing in a telephone directory, paying bills, and writing checks.

While medication management has been identified as a difficult task for older adults (Henriques et al., 2012; Lam et al., 2011; MacLaughlin et al., 2005; Owsley et al., 2001; Skomrock \& Richardson, 2010; Windham et al., 2005; Yasein et al., 2013), only one participant in this dissertation study indicated via the demographic information sheet that this was a difficult task. However, of the three occupational tasks in this dissertation study, sorting pills required the greatest amount of time among all groups of participants. The established link between time and effort (Zijlstra, 1993) points to this task being a difficult task for participants, but perhaps participants did not recognize or want to admit having difficulty with medication management. Further, it demonstrates the need to better understand occupational tasks that older adults find difficult and how they define and describe difficulty as well as the effect of lighting on the performance of 
occupational tasks, including whether optimal lighting levels should be recommended based on person factors, occupation factors or both.

Reading a prescription label. For the occupational task of reading a prescription label, bottles were obtained from a national chain pharmacy. Each amber colored bottle had a standard white label adhered to it, and while the words on each label were of similar reading level, they were different on each bottle, necessitating that participants read each label carefully. Completing the task took the greatest mean time under the medium lighting level $(M=127 \mathrm{fc})$ and the least mean time under the low lighting level $(M=103 \mathrm{fc})$. The difference in these two lighting levels was approximately $20 \mathrm{fc}$. The additional lighting for the medium lighting level was provided by a floor lamp placed to the right of the participant. This decision was made based on recommended standards (IESNA, 2010). However, as previously mentioned, it is not known whether the addition of this lamp was a hindrance to participants or cast glare or a shadow on the prescription label.

The fact that reading the prescription label took the least amount of overall mean time under the low lighting level was somewhat surprising, given that higher lighting levels are recommended for older adults when performing specific tasks, such as reading. Nonetheless, optimal reading performance at lower lighting levels is supported in the literature as Izso, Lang, et al. (2009) found participants were more accurate in NVT performance under lighting levels of approximately 1300 lux (130 fc), a level that corresponds to the medium lighting level in the dissertation study. However, the lowest lighting level in this study $(M=103)$ aligned with IESNA's recommendation of $100 \mathrm{fc}$ for older adults when reading (IESNA, 1998). Of note, the difference in mean 
completion time at each lighting level varied very little with the difference in low $(M=$ 9.52) and high lighting $(\mathrm{M}=9.86)$ being 0.34 seconds and the difference in high $(M=$ 9.86) and medium lighting $(M=11.80)$ being 1.94 seconds. As previously mentioned, it is possible that movement of participants or movement of the prescription bottle in the hands of participants cast a glare or shadow on the label when performing the task under the medium and high lighting levels. Also, participants were handed the prescription bottle and held it in hand while reading it. Because participants were not restrained from moving, it is possible that as they positioned the bottle to read it that they did so in a manner that prevented them from receiving the maximum benefit of the additional lighting or that they sensed glare from the additional lighting and positioned themselves out of the light in an attempt to avoid glare.

Several studies examining reading and NVT performance in older adults with and without visual impairment have demonstrated better results under higher lighting levels. However, variation existed in how researchers defined "higher" lighting levels in each of these studies with higher lighting levels ranging from 800 lux to 7500 lux ( 80-750 fc) (Bowers et al., 2001; Davis \& Garza, 2002; Eldred, 1992; Evans et al., 2010; Fosse \& Valberg, 2004; Haymes \& Lee, 2006; Izso, Laufer, et al., 2009). Further, participants' results were often individualized and varied, depending on the task, but in subjective ratings, older adults often preferred higher lighting levels (Davis \& Garza, 2002; Evans et al., 2010; Izso, Laufer, et al., 2009).

It is difficult to compare the results of the dissertation study with previous studies because of the variation in lighting levels utilized and the fact that other studies have not utilized measureable occupational tasks. Additionally, most studies investigating lighting 
and performance have utilized participants with eye disease, particularly ARMD. Very few researchers have investigated the performance of older adults with normal agerelated changes in vision as the dissertation study did.

Sorting pills. Sorting pills seemed to be the most tedious occupational task for participants based on the fact that it required the greatest mean completion time of the three tasks. For the pill sorting task, participants were required to sort 20 pills into four different sets. All three groups completed each subsequent trial in less time than the previous trial, which seems to suggest a practice effect. The investigator attributes this possible difference in part to the physical properties of the sets of pills. Each set contained one pill that was slightly larger than the others. While very close in size, the other pills differed in the presence or absence of an imprint and the type of imprint. It is possible that each subsequent trial was completed in less time because participants learned to sort by size and look for the presence or absence of imprints. This pattern of sorting could then be carried over into the next set of pills. Another factor that may have contributed to a possible practice effect was participants noticing that they were not penalized for inaccuracies. The investigator counted the pills after each trial but did not record the number sorted correctly as this information was not utilized as part of this dissertation study.

For this occupational task, the results were more varied among the groups with no particular lighting level appearing to lead to superior or inferior performance. However, when comparing the overall mean at each light level, this task was completed the quickest under the high lighting level and required the greatest amount of time under the medium lighting level. The amount of time required for this task seems to support the 
findings of previous researchers and demonstrate a degree of difficulty for participants. Medication management is an occupation that has been identified as difficult for older adults (Henriques et al., 2012; Lam et al., 2011; MacLaughlin et al., 2005; Owsley et al., 2001; Skomrock \& Richardson, 2010; Windham et al., 2005; Yasein et al., 2013), and distinguishing white and gray-toned colors is particularly difficult (Evans et al., 2010; Skomrock \& Richardson, 2010). Although only one participant in this dissertation study indicated via the demographic information sheet difficulty with medication management, the results of this study support previous research identifying medication management as a difficult task for older adults. The investigator believes this is a task worthy of further research as previous studies have not examined the effect of lighting on medication management, and it would behoove us to better understand the difficulty older adults experience as well as their perception of the difficulty experienced with this occupational task.

Sorting socks. The task of sorting dark socks was selected because sorting clothing by color is a difficult task for older adults (Barstow et al., 2011; Brunnstrom et al., 2004; Cook, 2009; Owsley et al., 2001), especially darker colors (Noell-Waggoner, 2004). Each set of socks, which contained black, navy, dark gray, and dark brown socks, was similar; therefore, participants had to examine each sock carefully to determine its color.

Unlike other occupational tasks in which performance required the greatest amount of time under the medium lighting level, sorting socks required the greatest amount of time under the low lighting level in all three groups regardless of the order. However, when comparing the overall means at each lighting level, sorting socks 
required the least amount of time under the medium lighting level and the greatest amount of time under the low lighting level although the mean difference in medium and high lighting was 0.25 seconds, a relatively small difference. While completion took the greatest amount of time for each group under the low lighting level for this task, Groups 1 and 2 required the least amount of time under the medium lighting level, and Group 3 required the least amount of time under the high lighting level. The fact that sorting socks required the most time under the low lighting level suggested that increased lighting levels were beneficial to participants as they performed this task.

As one ages, darker colors are difficult to differentiate (Noel-Waggoner, 2004), and cone cells, which provide color vision, are ineffective at lower light levels (Fosse \& Valberg, 2004; National Framework for Energy Efficiency, 2009). Also, age-related changes, including yellowing of the lens, make colors less vivid, and distinguishing colors in the blue range is difficult (Arditi, 2005; Boyce, 2003; Nolan, 2002; Oklahoma Geriatric Education Center, 2009; Salvi et al., 2006; Sekular \& Sekular, 2000; Watson, 2001). Given the number of participants who reported having or having had cataracts, it is not surprising that participants would have difficulty distinguishing the colors of the dark socks. For this task, it appears the additional lighting allowed for greater clarity in color discrimination, and unlike the prescription label and pills, the texture of the socks likely did not produce glare. These results appear to support the recommendation for utilization of more intense light, but additional lighting must be directed in order to avoid glare (Christenson, 1990). While participants in the dissertation study sorted socks more quickly under the medium lighting level rather than the high lighting level, the mean difference in the medium and high lighting levels was 0.25 seconds. Therefore, the 
results suggested that lighting levels do affect older adults' performance when completing this occupational task.

The total. Combining the completion time for each occupational task yielded a total time for the three tasks. The results of the total time for the three tasks varied by group, perhaps because the results of each task varied. Regardless of the lighting level, each group required less total time to complete the three tasks with each subsequent trial. However, sorting pills was the only occupational task for which less time was required for each subsequent trial in each group. Therefore, the results of that one task likely contributed to evidence of a practice effect in the total time for the three tasks. When comparing overall means at each lighting level, the total time required for the three occupational tasks was the least with the high lighting level and the greatest with the medium lighting level. This finding lends support to the research question, which questions the effect of lighting on older adults' performance of occupational tasks that are visually challenging and seems to suggest that when considering occupational task performance as a whole, more light leads to better performance.

The RSME. Evaluating the overall mean score on the RSME under each lighting level indicated that participants felt they exerted the least amount of effort while completing the occupational tasks under the high lighting level, and they reported the greatest amount of effort under the medium lighting level. For two of the three tasks, the mean completion time for participants was greatest under the medium lighting level as well as the total time for the three tasks. These results lend support to the research question, which questions the effect of lighting on older adults' perceived effort when performing visually challenging occupational tasks. However, participants in Groups 1 
and 3 rated less effort with each subsequent task, evidence that suggested a possible practice effect in those two groups.

When examining the results of the RSME, it is important to note that on a scale ranging from 0 to 150 with markers ranging from not effortful to awfully effortful along the scale, the mean range of participant scores was relatively low, ranging from 11.46 to 16.70. These scores seem to imply that participants did not find the occupational tasks required much effort. However, considering that there is an established association between time and effort (Zijlstra, 1993), the results on the RSME should be considered in terms of the time required to complete the occupational tasks. The fact that in general Group 1 required the greatest time to complete most tasks and that of the three groups, they also consistently rated the most effort, seems to support the relationship of time and effort.

Another point that must be considered is whether effort is the appropriate term and mechanism for measuring the subjective experience of the participants while performing the visually challenging tasks. Effort is difficult to define and measure (Yeo \& Neal, 2004). However, through the years, other terms, such as difficulty, have been studied in an attempt to understand the subjective experiences of older adults, and studying the relationship between time and effort has been recommended (Porter, 2007). Zijlstra (1993) developed the RSME for the purpose of measuring mental effort, and mental effort seems to be an appropriate concept for describing the experience of an older adult while performing visually challenging tasks under varying lighting levels. For this purpose, the RSME was selected over other scales that utilize factors, such as physiological responses, completion time, and subjective rating scales to measure effort 
(de Morree \& Marcora, 2010; Fraser et al., 2010; Geruschat \& Turano, 2007; LewisEvans et al., 2010). One must consider the results of this dissertation study in terms of the sample population. The participants were all well-educated residents of an independent living community and, therefore, may have been reluctant to admit that they had difficulty with any of the occupational tasks. Because scores on the RSME were so low, yet completion time seems to indicate the tasks did require some degree of effort, further research is recommended to better understand the subjective experiences of older adults during occupational performance and to better understand older adults' perception of effort and the relationship between time and effort, vision and effort, and effort and occupational performance.

\section{The Theoretical Context}

As previously noted, studies of vision and performance as well as studies of the home environment lack a theoretical perspective (Gitlin, 2003; Owsley, 2011). Because the PEO model acknowledges that a change in the environment can affect occupational performance, it was the theoretical context chosen for this dissertation study. Lighting is an important aspect of the environment that affect occupational performance, yet it has received little attention from occupational therapists or in the occupational therapy literature (Gendeman et al, 2010; Perlmutter, 2012; Perlmutter et al., 2013.). However, in order to improve occupational performance through environmental modifications, such as changes in lighting, we need a better understanding of the optimal lighting levels. This dissertation study suggested that lighting may affect occupational performance because in a setting in which lighting was the only manipulated variable, changes in occupational performance did occur. In addition to objective performance, the PEO model also 
addresses subjective performance, and in this study, the subjective aspect of occupational performance was measured by asking participants to rate their perceived effort while performing occupational tasks. The descriptive statistics suggested that lighting may affect older adults' perceived effort while performing visually challenging tasks because in an environment in which lighting was the only manipulated variable, changes in effort were reported.

\section{Conclusions}

One goal of this dissertation study was to investigate the effect of lighting on older adults' ability to perform selected occupational tasks that are visually challenging. While statistical significance could not be determined due to a violation of assumptions, the descriptive statistics do suggest that lighting levels affected the performance of older adults while completing visually challenging tasks. When examining the mean time required to complete the occupational tasks under the different lighting levels, the time varied for each occupational task. There was no clear evidence that all the occupational tasks were completed in less time under a certain lighting level. When examined individually by looking at mean completion times for each lighting level, each occupational task was completed most quickly at a different lighting level with each lighting level represented. Reading a prescription label required the least mean time under the low lighting level while sorting socks required the least mean time under the medium lighting level, and sorting pills required the least mean time under the high lighting level (see Table 16). In general, most tasks required the greatest amount of time to complete under the medium lighting level. The research question posed whether lighting levels affected performance of older adults for visually challenging tasks, and the 
results of this dissertation study suggested that lighting levels may affect older adults' performance on visually challenging tasks.

A second goal of this study was to investigate the effect of lighting on older adults' perceived effort when performing selected occupational tasks that are visually challenging. The greatest amount of effort was reported under the medium lighting level and the least amount of effort was reported under the high lighting level. The research question posed whether lighting levels affected perceived effort of older adults while performing visually challenging tasks. While statistical significance could not be determined due to a violation of assumptions, descriptive statistics suggested that lighting levels affected the perceived effort of older adults when completing visually challenging tasks.

As a general observation from the data, occupational tasks required the greatest time to complete and required the greatest amount of perceived effort under the medium lighting level. Overall, Group 1 required more time to complete the occupational tasks and reported greater effort when performing the tasks. These results seem to suggest a relationship between time and effort as noted in previous research (Zijlstra, 1993). Because participants in Group 1 were older and had poorer vision, these are factors that could have influenced the results and deserve consideration in future studies. Also, evidence points to a practice effect for one of the tasks (sorting pills) as well as the total of the three tasks and the RSME. However, because there was so much variation in completion times of the occupational tasks, general conclusions and recommendations should be viewed cautiously. While all tasks were visually challenging, they each presented the participant with a different challenge, for example, reading or sorting by 
color. As other researchers have noted, lighting is not one size fits all (American Lighting Association, 2011; Halloin, 1995; Perlmutter, 2012; Young, 2012). Perhaps consideration should be given to lighting that can be adjusted to meet the demands of the task and/or the demands of the person. However, in order to successfully meet the demands, additional research regarding optimal lighting levels, including the objective standards and subjective preferences of older adults, needs to be conducted.

Furthermore, future studies need to be guided by a theoretical perspective. Additionally, effort deserves further consideration by occupational therapists, particularly as it relates to occupational performance. We need to better understand the objective and subjective experiences of older adults in order to design client centered interventions and recommend environmental modifications, particularly changes in lighting, which enhance occupational performance.

\section{Implications for Occupational Therapy Practice}

The results of this dissertation study have several implications for occupational therapy practice. First, the dissertation study lends preliminary support to the assumption that lighting affects occupational performance in older adults, which is important because as people age, they spend more time indoors (Horgas et al., 1998) and are more dependent on the environment (IESNA, 1998). Consequently, it is recommended that occupational therapy practitioners in home health practice and those conducting home evaluations include an assessment of lighting in their evaluation measures. To date, this practice has been rare (Gendeman et al., 2010; Perlmutter, 2012; Perlmutter et al., 2013); however, Perlmutter (2013) recently developed a home lighting assessment. Occupational therapy practitioners need to be direct in their approach to addressing 
lighting in the home, observing and asking questions to best understand older adults' experiences with home lighting and its effects on daily living and task performance. However, they must be careful in their approach. Because age-related visual changes occur gradually (Rizzo, 2007; Salvi et al., 2006), changes in performance may be unrecognized, or older adults may be hesitant to admit difficulty with performance or with inadequate lighting in their homes. Because older adults may not recognize poor lighting conditions, it is important that occupational therapy practitioners not only interview older adult clients regarding lighting and occupational performance but also that they measure the lighting conditions in the home and observe performance under those lighting conditions. Additionally, occupational therapy practitioners must strive to build a rapport with older adult clients in hopes that older adults will understand the value in the recommendations that are made.

In addition to measuring lighting in homes, occupational therapy practitioners can educate older adults about optimal lighting levels and discuss the importance of optimal lighting to occupational performance and ways to achieve optimal lighting in the home. However, occupational therapy practitioners first will need to be knowledgeable about lighting and its effects in order to assess and educate older adults regarding lighting needs in the home. Older adults are often unaware of their lighting needs (Bakker et al., 2004; Lindner et al., 2001), and they use lighting that is suboptimal (Bakker et al., 2004). Occupational therapy practitioners need to understand the implications of lighting in the environment and the effects on occupational performance and be educated in lighting practices that will benefit older adults in their home environments. Until recently, lighting is a topic that has received little attention in occupational therapy (Gendeman et 
al., 2010; Perlmutter et al., 2013). However, it is hoped that this dissertation study will contribute to the limited body of knowledge about home lighting, which is a very relevant safety and occupational performance issue for the elderly.

Occupational therapy practitioners also have the opportunity to educate and work with architects, builders, remodelers, interior designers, and others in the design of lighting in existing and new homes and retirement communities. Designing lighting that is wisely placed, flexible, and adjustable could potentially provide for optimal occupational performance and safety in the home. Further, lighting fixtures that utilize lamps (bulbs) of the appropriate and recommended lumens and color temperature are important to occupational performance as well. As suggested in this dissertation study, lighting does potentially affect older adults' performance for tasks requiring near vision. While the value of night lights (Cumming et al., 1999) and lighting in stairways (Simpson \& Tarrant, 1983) has been recognized, also important is the lighting design in areas of the home, such as closets, laundry rooms, kitchens, and other areas, in which occupational tasks, such as laundry, clothing selection, and medication management occur. Lighting is one aspect of the environment that is rather easily modified and has the potential to contribute to older adults' ability to age in place as they desire (Gitlin, 2003; Gitlin et al., 2001).

\section{Implications for Future Research}

Because lighting research in occupational therapy is very limited, the implications for future research are numerous. It is hoped that this dissertation study will serve as a starting point for future research for lighting and occupational performance. Additional research is needed in order to better understand the lighting needs of older adults as well 
as to determine if current recommended lighting standards are adequate. While environmental factors are often more easily changed than person factors, it is important to know specifically which lighting levels and changes to lighting are most beneficial (Cooper et al., 2005). Further, because many lighting studies have taken place outside the United States, additional research is needed to better understand the lighting conditions in North American homes in order to address current inadequacies and barriers that exist.

Future studies investigating the effect of lighting on occupational performance are warranted, and several procedural changes are recommended. First, monitoring the number of errors on the occupational tasks is recommended. In this study, participants' completion time on each occupational task was recorded, but errors were not recorded. Perhaps participants noticed errors were not being recorded, which may have influenced their performance, particularly in the later trials. Participants may have been more focused on timely completion of the occupational tasks rather than the accuracy of their performance. Also, in future studies, consideration should be given to the position of individuals as they complete the occupational tasks (leaning forward or backward in the chair and toward or away from the light source) and the objects being viewed (distance at which participant held objects from their eyes) in relation to the lighting source. Nevertheless, movement during occupational performance is normal and expected and was observed during this dissertation study. While sitting in the chair in front of the task, participants were observed weight shifting, leaning forward, backward and side to side, during task performance. Additionally, completion of the occupational tasks required participants to manipulate the objects, and they were observed moving the objects closer 
to or further away from their eyes as well as the light source. However, preventing movement or prescribing placement of objects during task performance would create control, which is unnatural and not desired in a study of occupational performance. The issue that does need to be studied and better understood is optimal placement of objects and persons in relation to light sources during occupational performance. Although placed according to recommended standards (IESNA, 2010), it is possible that placement of additional lamps created shadows and/or glare that affected performance during this dissertation study.

Another factor to consider is the types of lamps and fixtures utilized. The lamps and fixtures were chosen for this dissertation study because they were either already present in the testing room or were accessible through local retailers. The color temperature and lumens of the bulbs were of recommended standards for older adults (IESNA, 2009; Washington State Department of Ecology, n.d.). However, other bulbs, such as LED, are presently more expensive, may become more predominant due to energy efficiency (American Lighting Association, 2002) and should be considered in future studies. Also, while natural lighting was not a factor in this dissertation study, it is a factor that may need consideration in future studies as it is present in residential homes.

This dissertation study utilized residents of one retirement center, and the study involved only three occupational tasks; therefore, it is recommended that future studies utilizing participants from other settings and different occupational tasks should be considered. While presenting the challenge of more variables to control, future studies of older adults' occupational performance could be conducted in homes or other natural environments. 
An additional procedural change for future consideration is the assignment of participants to groups. Because the study took place in a retirement community where many residents knew one another, the investigator desired random assignment to groups in hopes of preventing participants from discussing the order of lighting with future participants. However, the order of the lighting did not seem to be a factor noticed by participants. Because the investigator had no way of determining in advance how many residents would agree to participate and meet the inclusion criteria, random assignment resulted in uneven groups with one group having nearly twice as many participants as another. For future studies, assignment of participants to groups is recommended in order to ensure more equally sized groups.

Furthermore, it is recommended that research for lighting in occupational performance be grounded in occupational therapy theory. When person factors, such as age-related visual changes, cannot be corrected (Noell-Waggoner, 2004), the environment can be altered, thereby improving occupational performance as per the PEO model. For this reason, the PEO model is an excellent theory on which to base future studies for lighting and occupational performance. The PEO model considers both objective and subjective aspects of occupational performance, which is an important consideration when studying older adults and the effect of environmental factors, such as lighting (McColl \& Pollock, 2005).

Finally, this dissertation study serves as a starting point for investigating the relationship between vision, effort, the environment, and occupational performance. Effort is a difficult term to define and measure (Yeo \& Neal, 2004), and it has not been addressed a great deal in the literature, particularly in occupational therapy literature. 
Older adults struggle to define and describe the difficulties they encounter when performing daily living skills (Porter, 2007). They may not want to admit performing everyday tasks now requires more effort, or they may not recognize or acknowledge that they are experiencing difficulty. Only a few participants in this dissertation study reported difficulty with task performance when completing the study's initial questionnaire, and participants reported very little effort when performing the occupational tasks as evidenced by scores on the RSME. It is not known whether they did not feel as though they exerted effort, did not want to admit the occupational tasks required effort, or if effort is not the most appropriate construct for describing and defining their experiences with completing the occupational tasks under different lighting levels. However, while the three groups were fairly equitable in terms of age, vision, and cognition, Group 1 participants (the oldest and had the poorest vision) reported the most effort and often took the most time to complete the occupational tasks. Further, Group 2 participants (the youngest and had the best vision and highest cognitive scores) performed the quickest on two of the three tasks and reported the least effort. Thus, the relationship between aging, vision, lighting, and effort needs to be further studied and understood. Occupational therapy practitioners, therefore, must be intentional in discussing effort and task performance difficulty during client evaluations and interventions in an attempt to better understand how effort influences occupational performance. Additionally, future research must continue in order to better define and understand effort and its effect on older adults' occupational task performance. Qualitative studies seeking older adults' insights on difficulties they experience and opinions on their experiences while performing visually challenging tasks would be 
particularly beneficial in generating future research hypotheses that address perceived effort and occupational performance (Portney \& Watkins, 2009).

\section{Limitations}

There are several limitations to the study. First, the fact that the assumption of complete cells was violated, preventing the use of RMANOVA and limiting the results achieved with the three-way ANOVA is a limitation. A future study utilizing RMANOVA would be most appropriate. However, focusing less on randomization of factors is advised as is re-evaluating the lighting factor to ensure each lighting level is achieved in each trial is important. In this dissertation study, random assignment of members to groups resulted in uneven groups. The rationale for random assignment was to prevent participants in the close-knit retirement community from discussing lighting order with future participants in the study. However, this concern did not seem to be a factor in the dissertation study. In future studies, it is recommended that members be assigned to a group in order to ensure a more even number of participants in each group. Also, to meet the assumption of complete cells as required for RMANOVA, each lighting level must be represented in each trial. In the dissertation study, the low light level was not represented in Trial 2, and the medium light level was not represented in Trial 3. Structuring a future study as depicted in Table 16 ensures each lighting level (low, medium, high) is represented in each of the three trials. Utilizing this design will allow for completion of the RMANOVA to address the null hypotheses that changing light levels will result in no significant changes in older adults' performance of visually challenging tasks as measured by time to complete tasks, and changing light levels will 
result in no significant changes in perceived effort of older adults while performing visually challenging tasks as measured on a self-report scale.

Additionally, while the occupational tasks selected for the study seemed appropriate and sufficiently challenging, further consideration needs to be given to measures that will minimize the risk of a practice effect when performing the occupational tasks. Utilizing testing materials that are less similar will perhaps minimize this risk with the realization that doing this modification imposes other limitations into the study. Perhaps the researcher interviewing participants between trials to collect questionnaire responses would provide participants an opportunity to rest and momentarily redirect their attention away from the routine pattern of the occupational tasks while also allowing the researcher to collect more thorough and accurate demographic information.

Table 16

Recommended Groups, Trials, and Lighting Levels

\begin{tabular}{cccc}
\hline & \multicolumn{3}{c}{ Lighting level } \\
\cline { 3 - 4 } Group & Trial 1 & Trial 2 & Trial 3 \\
\hline 1 & Low & Medium & High \\
2 & High & Low & Medium \\
3 & Medium & High & Low \\
\hline
\end{tabular}

Another limitation of the study was the small sample size. While there were over 200 residents within the retirement community, only 31 , or approximately $15 \%$, volunteered for the study. The weather presented unforeseen challenges during the data collection period and perhaps contributed to the small sample size. Also contributing to the size of the sample could have been the health status and capability of older adults in 
the retirement community. Additionally, some residents may have just been reluctant to be involved in a research study.

Further, the dissertation study was limited in that some participant information was obtained via self-report from the participants. The investigator was relying on participant's awareness and accurate reporting of the demographic information. Most notably, the information regarding the presence of eye disease provided data that was somewhat conflicting with some participants who indicated they had cataracts yet no eye disease.

The dissertation study was limited to the selected occupational tasks and the specific lighting levels. The tasks and lighting levels were selected based on a thorough review of the literature, and the investigator selected lamps that were readily available to the older adult population at national chain retailers. While the placement of the lamps was selected by the investigator and consistent across all participants, the movement of the participants while performing the occupational tasks was not controlled for. This movement could have potentially created glare or shadows on the occupational tasks that affected performance. However, the investigator's goal was to utilize occupational tasks that were visually challenging, lighting levels that met recommended standards, and lamps that could easily be acquired by older adults in the community. These goals were accomplished within the dissertation study. Further, it should be noted that when performing everyday tasks, it is natural for older adults to move, make postural adjustments, and manipulate the materials being utilized in the task. For this reason, requiring participants to maintain a static position was not practical nor the desire of the investigator. 
Finally, because this dissertation study utilized a sample of convenience from a retirement community in a large metropolitan area in Arkansas and was comprised of participants who are elderly, well-educated, and pay to live in the community, the results cannot be generalized to all populations. Additionally, because the participants were aware that the investigator was conducting the research in pursuit of a $\mathrm{PhD}$ in an arena in which several participants reported advanced education, this fact may have influenced residents' decision to participate.

\section{Recommendations}

This dissertation served as a preliminary examination of the impact of lighting on performance of visually challenging tasks in older adults and the effect of lighting on perceived effort of older adults when performing visually challenging tasks. There is a need for additional education of occupational therapy practitioners regarding lighting and the lighting needs of older adults so that occupational therapy practitioners can address lighting needs with clients for the purpose of improving occupational performance and safety within the home. Occupational therapy practitioners who are knowledgeable about the lighting needs of older adults can provide this information to clients, empowering them to make informed choices regarding their lighting needs. Additionally, there is a need for occupational therapy practitioners to better understand the experiences and challenges older adults face during occupational performance, including their understanding and acknowledgement of effort expended during occupational performance. An enhanced understanding of effort will better equip occupational therapy practitioners in addressing the challenges of older adults in their homes and in designing interventions that support aging in place. 
In the future, the investigator recommends replication of the study with several adjustments. Adjustment of the lighting order in a manner that will allow for complete cells (see Table 16) is recommended. Slightly increasing the variation in the testing sets in an effort to decrease the potential of a learning effect is also recommended. For example, utilizing a variety of white and pastel pills rather than all-white ones may decrease the potential for a learning effect. Another recommendation is recording errors committed by participants for each occupational task. The investigator believes that the fact that participants became aware that errors were not being recorded may have contributed to a learning effect in the dissertation study by allowing participants to finish more quickly without fear of penalty for errors committed. Additionally, in this dissertation study, high contrast was provided for occupational task performance. For example, white pills were sorted against a black background. However, because not all occupational task performance occurs in environments of high contrast, it is recommended that future studies consider occupational task performance under varying levels of contrast.

The investigator believes the information collected via the questionnaire was appropriate and beneficial. However, in future studies, having the researcher collect and record the information from the participant rather than relying on the participant to record the information may be beneficial, eliminating conflicting self-reported information, such as recording both "no eye disease" and "cataracts." Additionally, having the researcher collect this information via interview in between trials would allow the participant time to rest and focus on information besides the occupational tasks, thereby perhaps decreasing the chances of a practice effect. Asking selected demographic questions after rather than 
before occupational task performance is also recommended. Doing so would allow the researcher to ask for clarification regarding participant answers if needed without compromising the integrity of the results. For example, in this dissertation study, one participant indicated that paying bills was difficult but did not indicate why the task was difficult. The investigator did not ask for clarification because probing for this information prior to task completion could have biased the results by alerting the participant to the fact that lighting and vision were factors being studied. If questions regarding difficulty were asked after occupational task performance, the researcher could ask for specifics about the nature of difficulty in order to learn if the source of difficulty is related to vision or lighting.

\section{Summary}

This quasi-experimental study utilized a repeated measures design to investigate the effect of lighting on older adults' performance of visually challenging tasks and the effect of lighting on older adults' perceived effort while performing the tasks. Lighting affects performance of older adults, but few researchers have studied the effect of lighting on the performance of functional tasks. Thirty participants who were residents of a retirement community in a large metropolitan area in Arkansas participated in the dissertation study. The results were described through descriptive statistics, and when comparing the three groups, differences were observed in performance on the occupational tasks as well as the perceived effort. In general, participants required longer to complete occupational tasks and reported more effort when utilizing medium light levels $(M=127 \mathrm{fc})$ provided by ambient lighting and a floor lamp and performed best 
under high lighting levels $(M=397 \mathrm{fc})$ provided by ambient lighting as well as a floor lamp and task lamp.

In conclusion, the findings of this dissertation study suggested that lighting may affect older adults' performance for occupational tasks that are visually challenging as well as their perceived effort when performing those occupational tasks. Additional research is needed to further explore the effect of lighting on older adults' occupational performance, including the quality, intensity, and placement of lighting in relation to occupational tasks. Further, occupational therapy practitioners must be educated regarding the importance of evaluating lighting in the homes of older adults and making recommendations that will enhance safety and occupational performance.

Also recommended is additional research that will broaden our understanding of older adults' perception of effort in relation to occupational task performance. Better understanding the impact of lighting on occupational task performance and the perceived effort of older adults during occupational task performance will contribute to the knowledge base and plans for aging in place, which so many adults desire to do. 


\section{References}

6 low cost or no-cost home modifications to avoid falls. (2015). Harvard Health Letter, $40,1,7$.

Adams, P., Kirzinger, W., \& Martinez, M. (2013). Summary health statistics for the U.S. population: National health interview survey 2012. National Center for Health Statistics. Vital Health Statistics, 10(259). Retrieved from http://www.cdc.gov/nchs/data/series/sr_10/sr10_259.pdf

Administration on Aging. (2014). A profile of older Americans: 2014. Retrieved from http://www.aoa.acl.gov/Aging_Statistics/Profile/2014/docs/2014-Profile.pdf

American Lighting Association. (2012). Lighting your life. Retrieved from http://www.lightrays-digital.com/lightrays/lightinglife2012?pg=1\#pg1

American Occupational Therapy Association. (2014). Occupational therapy practice framework: Domain and process (3rd ed.). American Journal of Occupational Therapy, 68, S1-S48. http://dx.doi.org/10.5014/ajot.2014.682006

Architectural Acoustics and Lighting. (n.d.). Lighting terminology. Retrieved from http://home.tir.com/ ms/lightingdefinitions/lightingdefinitions.html

Arditi, A. (2005). Enhancing the visual environment for older and visually impaired persons. Alzheimer's Care Quarterly, 6, 294-299.

Bakker, R., Iofel, Y., \& Lachs, M. (2004). Lighting levels in the dwellings of homebound older adults. Journal of Housing for The Elderly, 18, 17-27.

Barberger-Gateau, P., Rainville, C., Letenneur, L., \& Dartigues, J. (2000). Hierarchial model of domains of disablement in the elderly: A longitudinal approach. Disability and Rehabilitation, 22, 308-317.

Barbur, J., \& Konstantakopoulou, E. (2012). Changes in color vision with decreasing light level: Separating the effects of normal aging from disease. Journal of the Optical Society of America, 29, A27-A35.

Barstow, B., Bennett, D., \& Vogtle, L. (2011). Perspectives on home safety: Do home assessments address the concerns with vision loss? American Journal of Occupational Therapy, 65, 635-642.

Beckman, A., Parker, M., \& Thorslund, M. (2005). Can elderly people take their medicine? Patient Education and Counseling, 59, 186-191.

Berger, S., \& Kaldenberg, J. (2008). Health conditions associated with aging. In S. Coppola, S. Elliott, \& P. Toto (Eds.), Strategies to advance gerontology 
excellence: Promoting best practice in occupational therapy (pp. 199-222). Bethesda, MD: AOTA Press.

Berger, S., \& Porell, F. (2008). The association between low vision and function. Journal of Aging and Health, 20, 504-525.

Bodart, M., Roisin, B., Deneyer, A., \& D'Herdt, P. (2009, September). Establishment of innovation guidelines for the residential lighting from a relighting project. Paper session presented at the meeting of Lux Europa, Istanbul, Turkey. Retrieved from http://www.cstc.be/homepage/download.cfm?dtype=research\&doc=paper_244.pd f\&lang=en

Borell, L., Lilja, M., Sviden, G., \& Sadlo, G. (2001). Occupations and signs of reduced hope: An explorative study of older adults with functional impairments. American Journal of Occupational Therapy, 55, 311-316.

Bowers, A., Meek, C., \& Stewart, N. (2001). Illumination and reading performance in age-related macular degeneration. Clinical and Experimental Optometry, 84, 139147.

Boyce, P. (2003). Lighting for the elderly. Technology and Disability, 15, 165-180.

Brabyn, J., Haegerstrom-Portnoy, G., Schneck, M., \& Lott, L. (2000). Visual impairments in elderly people under everyday viewing conditions. Journal of Visual Impairment \& Blindness, 94, 741-744, 746-755.

Brown, M., \& Jacobs, D. (2011). Residential light and risk for depression and falls: Results from the LARES study of eight European cities. Public Health Reports, 126(Suppl. 1), 131-140.

Brunnstrom, G., Sorensen, S., Alsterstad, K., \& Sjostrand, J. (2004). Quality of light and quality of life-The effect of lighting adaptation among people with low vision. Ophthalmic and Physiologic Optics, 24, 274-280.

Capa, R.-L., Audiffren, M., \& Ragot, S. (2008). The interactive effect of achievement motivation and task difficulty on mental effort. International Journal of Psychophysiology, 70, 144-150.

Centers for Disease Control and Prevention. (2013). Important facts about falls. Retrieved from http://www.cdc.gov/HomeandRecreationalSafety/Falls/adultfalls.html

Charness, N., \& Dijkstra, K. (1999). Age, luminance, and print legibility in homes, offices, and public places. Human Factors: The Journal of Human Factors and Ergonomics Society, 41, 173-193. 
Christiansen, C., \& Baum, C. (2005). The complexity of human occupation. In C. Christiansen, C. Baum, \& J. Bass-Haugen (Eds), Occupational therapy: Performance, participation, and well-being (pp. 2-24). Thorofare, NJ: SLACK, Inc.

Christenson, M. (1990). Aging in the designed environment. New York, NY: The Haworth Press.

Chu, Y., Kaldenberg, J., \& Huefner, K. (2009). The elder's right to sight collaborative: A new model of eye care delivery for the elderly. Optometry, 80, 651-656.

Cognitive (n.d.). In Merriam-Webster's online dictionary. Retrieved from http://www.merriam-webster.com/dictionary/cognitive

Cognition (n.d.). In Merriam-Webster's online dictionary. Retrieved from http://www.merriam-webster.com/dictionary/cognition

Cohen-Mansfield, J., Shmotkin, D., Blumstein, Z., Shorek, A., Eyal, N, \& Hazan, H. (2013). The old, old-old, and the oldest old: Continuation or distinct categories? An examination of the relationship between age and changes in health, function, and well-being. International Journal of Aging and Human Development, 77, 3757.

Colenbrander, A. (n.d.a). The basic low vision exam. Retrieved from https://www.dropbox.com/s/3h91a01qv3mxt4u/Low\%20Vision\%20Exam.pdf?dl $=0$

Colenbrander, A. (n.d.b). The Colenbrander Low Vision Measurement System Retrieved from http://legacy.ski.org/Colenbrander/Images/LV_system.pdf

Conservation Technology of Illinois. (2014). Residential lighting design guide. Retrieved from http://www.contechlighting.com/sites/default/files/contechresidentaillightingguide .pdf

Cook, G. (2009). Domestic lighting for people with sight loss-The real provision of general and task light in 57 homes. Light \& Engineering, 17, 32-38.

Cooper, B., Letts, L., Rigby, P., Stewart, D., \& Strong, S. (2005). Measuring environmental factors. In M. Law, C. Baum, \& W. Dunn (Eds.), Measuring occupational performance: Supporting best practice in occupational therapy $\left(2^{\text {nd }}\right.$ ed., pp. 315-346). Thorofare, NJ: SLACK, Inc.

Coppola, S. (2008). A transactional approach to understanding meanings and benefits of occupation in older adulthood. In S. Coppola, S. Elliott, \& P. Toto (Eds.), 
Strategies to advance gerontology excellence: Promoting best practice in occupational therapy (pp. 279-318). Bethesda, MD: AOTA Press.

Cornelissen, F., Bootsma, A., \& Kooijman, A. (1995). Object perception by visually impaired people at different light levels. Vision Research, 35, 161-168.

Csikszentmihalyi, M. (1997). Finding flow. Psychology Today, 30, 46-48, 70-71.

Cullinan, T., Silver, J., Gould, E., \& Irvine, D. (1979). Visual disability and home lighting. The Lancet, 1, 642-644.

Cumming, R., Thomas, M., Szonyi, G., Salkeld, G., O'Neill, E., Westbury, C., \& Frampton, B. (1999). Home visits by an occupational therapist for assessment and modification of environmental hazards: A randomized trial of fall prevention. Journal of the American Geriatrics Society, 47, 1397-1402.

Davis, R., \& Garza, A. (2002). Task lighting for the older adult. Journal of the Illuminating Engineering Society, 31, 20-32.

December Communications. (n.d.). Color terms. Retrieved from http://www.december.com/html/spec/colorterms.html

De Morree, H., \& Marcora, S. (2010). The face of effort: Frowning muscle activity reflects effort during a physical task. Biological Psychology, 85, 377-382.

de Waard, D. (2006). The measurement of drivers' mental workload (Doctoral dissertation). Retrieved from http://home.zonnet.nl/waard2/dewaard1996.pdf

Dudgeon, B., Hoffman, J., Ciol, M., Shumway-Cook, A., Yorkston, K., \& Chan, L. (2008). Managing activity difficulties at home: A survey of Medicare beneficiaries. Archives of Physical Medicine and Rehabilitation, 89, 1256-1261.

Efficiency. (n.d.). In Merriam-Webster's online dictionary. Retrieved from http://www.merriam-webster.com/dictionary/efficiency

Effort. (n.d.). In Merriam-Webster's online dictionary. Retrieved from http://www.merriam-webster.com/dictionary/effort

Eldred, K. (1992). Optimal illumination for reading in patients with age-related maculopathy. Optometry and Vision Science, 69, 46-50.

Energy Trust of Oregon. (2013). Footcandle light guide. Retrieved from https://www.lightingdesignlab.com/sites/default/files/pdf/Footcandle_Lighting\%2 0Guide_Rev.072013.pdf 
Environmental Protection Agency. (1995, June). EPA 430-R-94-001 Green lights program: Lighting upgrade. Washington, DC: Author. Retrieved from http://nepis.epa.gov/Exe/ZyPDF.cgi/9100NXVP.PDF?Dockey=9100NXVP.PDF

Eperjesi, F., Maiz-Fernandez, C., \& Bartlett, H. (2007). Reading performance with various lamps in age-related macular degeneration. Ophthalmic and Physiological Optics, 27, 93-99.

Erkal, S. (2010). Home safety, safe behaviors of elderly people, and fall accidents at home. Educational Gerontology, 36, 1051-1064.

Esposito, F., Gendolla, G., \& Van der Linden, M. (2014). Are self-efficacy beliefs and subjective task demand related to apathy in aging? Aging and Mental Health, 18, 521-530.

Evans, B., Sawyer, H., Jessa, Z., Brodrick, S., \& Slater, A. (2010). A pilot study of lighting and low vision in older people. Lighting Research and Technology, 42, 103-119.

Extech Instruments. (2013). Extech Instruments model 401027 pocket foot candle light meter user manual. Nashua, NH: Author. Retrieved from http://www.extech.com/resources/401027_UM.pdf

Fange, A., \& Iwarsson, S. (2005). Changes in ADL dependence and aspects of usability following housing adaptation: A longitudinal perspective. American Journal of Occupational Therapy, 59, 296-304.

Faul, F. (2014). G*Power, version 3.1.9. Kiel, Germany: Department of Psychology, Christian-Albrechts University.

Federal Trade Commission. (2010). Coming in 2011: New labels for light bulb packaging. Retrieved from http://www.ftc.gov/news-events/pressreleases/2010/06/coming-2011-new-labels-light-bulb-packaging

Federal Trade Commission. (2011). Consumer information: Shopping for light bulbs. Retrieved from https://www.consumer.ftc.gov/articles/0164-shopping-light-bulbs

Figueiro, M. (2001). Lighting the way: A key to independence. Retrieved from http://www.lrc.rpi.edu/programs/lightHealth/AARP/pdf/AARPbook3.pdf

Fosse, P., \& Valberg, A. (2004). Lighting needs and lighting comfort during reading with age-related macular degeneration. Journal of Visual Impairment and Blindness, 98, 389-409.

Fraser, S., Gagne, J.-P., Alepins, M., \&Dubois, P. (2010). Evaluating the effort expended to understand speech in noise using a dual-task paradigm: The effects of 
providing visual speech cues. Journal of Speech, Language, and Hearing Research, 53, 18-33.

Gendeman, J., Perlmutter, M., \& Baum, C. (2010). Lighting assessment and intervention: The state of current practice. St. Louis, MO: Washington University Program in Occupational Therapy Scholar Day.

Geruschat, D., \& Turano, K. (2007). Estimating the amount of mental effort required for independent mobility: Persons with glaucoma. Investigative ophthalmology and Visual Science, 48, 3988-3994.

Gill, T., Williams, C., Robison, J., \& Tinetti, M. (1999). A population-based study of environmental hazards in the homes of older persons. American Journal of Public Health, 89, 553-556.

Gitlin, L. (2003). Conducting research on home environments: Lessons learned and new directions. The Gerontologist, 43, 628-637.

Gitlin, L., Mann, W., Tomits, M., \& Marcus, S. (2001). Factors associated with home environmental problems among community-living older people. Disability and Rehabilitation, 23, 777-787.

Gitlin, L., Winter, L., Dennis, M., Corcoran, M., \& Hauck, W. (2006). A randomized trial of a multicomponent home intervention to reduce functional difficulties in older adults. Center for Applied Research on Aging and Health Research Papers, 5. Retrieved from http://jdc.jefferson.edu/cgi/viewcontent.cgi?article=1005\&context=carah_papers

Gravetter, F., \& Wallnau, L. (2009). Statistics for the behavioral sciences ( $8^{\text {th }}$ ed.). Belmont, CA: Wadsworth.

Greene, D., Sample, P., \& Fruhauf, C. (2009). Fall prevention pilot: Hazard survey and responses to recommendations. Occupational Therapy in Healthcare, 23, 24-39.

Green, S., \& Salkind, N. (2008). Using SPSS for Windows and Macintosh: Analyzing and understanding data ( $5^{\text {th }}$ ed.). Upper Saddle River, NJ: Pearson.

Grue, E., Ranhoff, A., Noro, A., Finne-Soveri, H., Jensdottir, A., Ljunggren, G., . . . Jonsson, P. (2009). Vision and hearing impairments and their associations with falling and loss of instrumental activities in daily living in acute hospitalized older person in five Nordic hospitals. Scandinavian Journal of Caring Sciences, 23, $635-643$.

Haak, M., Ivanoff, S., Fange, A., Sixsmith, J., \& Iwarsson, S. (2007). Home as the locus and origin for participation: Experiences among very old Swedish people. 
Occupational Therapy Journal of Research: Occupation, Participation and Health, 27, 95-103.

Halloin, J. (1995). Application of current knowledge in lighting to the needs of the homebound aged. Journal of Long-Term Home Health Care, 14, 32-39.

Hall, R. (1998). With-in subjects design. Retrieved from https://web.mst.edu/ psyworld/experimental/within_subjects.html

Hamilton, T. (2010). Occupations and places. In C. Christiansen \& E. Townsend (Eds.), Introduction to occupation: The art and science of living (2nd ed., pp. 251-279). Upper Saddle River, NJ: Pearson.

Hayase, D., Mosenteen, D., Thimmaiah, D., Zemke, S., Atler, K., \& Fisher, G. (2004). Age-related changes in activities of daily living. Australian Occupational Therapy Journal, 51, 192-198.

Hayes, K. (2005). Designing written medication instructions: Effective ways to help older adults self-medicate. Journal of Gerontological Nursing, 36, 5-10.

Haymes, S., \& Lee, J. (2006). Effects of task lighting on visual function in age-related macular degeneration. Ophthalmic and Physiological Optics, 26, 169-179.

Henriques, M., Costa, M., \& Cabrita, J. (2012). Adherence and medication management by the elderly. Journal of Clinical Nursing, 21, 3096-3105.

Horgas, A., Wilms, H., \& Baltes, M. (1998). Daily life in very old age: Everyday activities as expression of successful living. The Gerontologist, 38, 556-568.

Illumenate. (2016). Lighting levels: Guidelines and definitions. Retrieved from http://www.illumenate.com/lightlevels.htm

Illuminating Engineering Society of North America (2009). Lighting your way to better vision (IES CG-1-09). New York, NY: Author. Retrieved from http://www.ies.org/PDF/Education/LightingForAgingEye.pdf

Illuminating Engineering Society of North America. (2010). Light in design: An application guide (IES CP-2-10). New York: NY: Author. Retrieved from http://lumaxlighting.com/wpcontent/uploads/2014/06/www.ies.org_PDF_Educati on_Li htInDesign.pdf

Illuminating Engineering Society of North America. (1998). Recommended practice for lighting and the visual environment for senior living. New York, NY: Author.

Illuminating Engineering Society of North America. (n.d.). Spectral power distribution. Retrieved from 
http://www.lrc.rpi.edu/education/learning/terminology/spectralpowerdistribution.a $\mathrm{sp}$

Innovative Lighting, Inc. (2013). Photopic and scotopic vision. Retrieved from http://www.innovativelight.com/led_facts/photopic--scotopic-vision/

Ishihara, K., Ishihara, S., Nagamachi, M., Osaki, H., \& Hiramatsu, S. (2004). Independence of older adults in performing instrumental activities of daily living (IADLs) and the relation of this performance to visual abilities. Theoretical Issues in Ergonomics Science, 5, 198-213.

Iwarsson, S., \& Isacsson, A. (1996). Housing standards, environmental barriers in the home and subjective general apprehension of housing situation among rural elderly. Scandinavian Journal of Occupational Therapy, 3, 52-61.

Izso, L., Lang, E., Laufer, L., Suplicz, S., \& Horvath, A. (2009). Psychophysiological, performance and subjective correlates of different lighting conditions. Lighting Research and Technology, 41, 349-360.

Izso, L., Laufer, L., \& Suplicz, S. (2009). Effects of dynamic lighting on the visual performance of older adults. Lighting Research and Technology, 41, 361-370.

Jacobs, K., \& Simon, L. (2015). Quick reference dictionary for occupational therapy $\left(5^{\text {th }}\right.$ ed). Thorofare, NJ: SLACK, Inc.

Kolanowski, A. (1992). The clinical importance of environmental lighting to the elderly. Journal of Gerontological Nursing, 18, 10-14.

Krusen, N. (2010, October 25). Home lighting: Understanding what, where, and how much. OT Practice, 15, 8-12.

Kukla, A. (1972). Foundations of an attributional theory of performance. Psychological Review, 79, 454-470.

Kuyk, T., \& Elliott, J. (1999). Visual factors and mobility in persons with age-related macular degeneration. Journal of Rehabilitation Research and Development, 36, 303-312.

Lam, P., Elliott, R., \& George, J. (2011). Impact of a self-administration of medications programme on elderly inpatients' competence to manage medications: A pilot study. Journal of Clinical Pharmacy and Therapeutics, 36, 80-86.

Langlois, J., Maggi, S., Harris, T., Simonsick, E., Ferrucci, L., Pavan, M., Sartori, L., \& Enzi, G. (1996). Self-report of difficulty performing functional activities identifies a broad range of disability in old age. Journal of the American Geriatrics Society, $44,1421-1428$. 
Law, M., Cooper, B., Strong, S., Stewart, D., Rigby, P., \& Letts, L. (1996). The personenvironment-occupation model: A transactive approach to occupational performance. Canadian Journal of Occupational Therapy, 63, 9-23.

Law, M., \& Dunbar, S. (2007). Person-environment-occupation model. In S. Dunbar (Ed.). Occupational therapy models for intervention with children and families (pp. 27-49). Thorofare, NJ: SLACK, Inc.

Law, M. (2002). Participation in the occupations of everyday life. American Journal of Occupational Therapy, 56, 640-649.

Lewis-Evans, B., De Waard, D., \& Brookhuis, K. (2010). That's close enough-A threshold effect of time headway on the experience of risk, task difficulty, effort, and comfort. Accident Analysis and Prevention, 42, 1926-1933.

Light meter. (n.d.). In Merriam-Webster's online dictionary. Retrieved from http://www.merriam-webster.com/dictionary/light\%20meter

Light. (n.d.) In Merriam-Webster's online dictionary. Retrieved from http://www.merriam-webster.com/dictionary/efficiency

Lin, S., Beck, A., Finch, B., Hummer, R., \& Master, R. (2012). Trends in US older adult disability: Exploring age, period and cohort effects. American Journal of Public Health, 102, 2157-2163.

Lindner, H., Rinnert, T., \& Behrens-Baumann, W. (2001). Illumination conditions of visually impaired people under private domestic conditions. Klinische Monatsblatter fur Augenheilkunde, 12, 774-781.

Ludwig, F. (2004). Occupation-based and occupation-centered perspectives. In K. Walker \& F. Ludwig (Eds.), Perspectives on theory for the practice of occupational therapy ( $3^{\text {rd }}$ ed., pp. 373-442). Austin, TX: Pro-Ed.

McColl, M., \& Pollock, N. (2005). Measuring occupational performance using a clientcentered perspective. In M. Law, C. Baum, \& W. Dunn (Eds.), Measuring occupational performance: Supporting best practice in occupational therapy $\left(2^{\text {nd }}\right.$ ed., pp. 80-91). Thorofare, NJ: SLACK, Inc.

Mackintosh,S., Fryer, C., \& Southerland, M. (2007). For falls sake: Older carers' perception of falls and fall risk factors. Internet Journal of Allied Health Sciences and Practice, 5, 3. Retrieved from http://ijahsp.nova.edu/articles/vol5num3/mackintosh.htm

MacLaughlin, E., Raehl, C., Treadway, A., Sterling, T., Zoller, D., \& Bond, C. (2005). Assessing medication adherence in the elderly. Drugs Aging, 22, 231-255. 
Marieb, E. (2001). Human Anatomy and physiology ( $5^{\text {th }}$ ed.). San Francisco, CA: Benjamin Cummings.

Meyers, J, \& Wilcox, D. (2011). Low vision evaluation. In M. Warren \& E. Barstow (Eds.). Occupational therapy interventions for adults with low vision (pp. 47-73). Bethesda, MD: AOTA Press.

Miller, S. (2001). Literature review: Workload measures. Iowa City, IA: University of Iowa. Retrieved from http://www.nadssc.uiowa.edu/publicationStorage/200501251347060.N01-006.pdf

Mogk, L. (2011). Eye conditions that cause low vision. In M. Warren \& E. Barstow (Eds.). Occupational therapy interventions for adults with low vision (pp. 27-46). Bethesda, MD: AOTA Press.

Moller, J. (2008, June). A design primer for the aging eye: Interior design features to consider when accommodating fading vision. Long-Term Living: For the Continuing Care Professional, 57, 24-28.

National Framework for Energy Efficiency. (2009). The basics of efficient lighting: A reference manual for training in efficient lighting principles. Retrieved from http://www.energyrating.gov.au/document/training-guide-basics-efficient-lighting

Nazarko, L. (2008). Falls Part 3: Environmental risk factors. British Journal of Healthcare Assistants, 2, 487-490.

Noell-Waggoner, E. (2004). Lighting solutions for contemporary problems of older adults. Journal of Psychosocial Nursing, 42, 14-20.

Nolan, D. (2002). Normal age-related vision loss and related services for the elderly. Retrieved from http://www.laurenscharff.com/research/donia/aging_vision_front.htm

Nylen, P., Favero, F., Glimme, S., Fahnehjelm, K., Eklund, J. (2014). Vision, light and aging: A literature overview on older-age workers. Work, 47, 399-412.

Oklahoma Geriatric Education Center. (2009). The aging eye: Normal physiological changes in the aging eye. Self-instructional modules in geriatric medicine $\left(5^{\text {th }}\right.$ ed.) Retrieved from http://www.ouhsc.edu/geriatricmedicine/Education/aging_eye/aging_eye.htm

Orellano, E., Colon, W., \& Arbesman, M. (2012). Effect of occupation- and activitybased interventions on instrumental activities of daily living performance among community dwelling older adults: A systematic review. American Journal of Occupational Therapy, 66, 292-300. 
Owsley, C., McGwin, G., Sloane, M., Stalvey, B., \& Wells, J. (2001). Timed instrumental activities of daily living tasks: Relationship to visual function in older adults. Optometry and Vision Science, 78, 350-359.

Owsley, C. (2011). Aging and vision. Vision Research, 51, 1610-1622.

PAR, Inc. (2012). Mini-Mental State Examination, $2^{\text {nd }}$ Edition (MMSE-2). Retrieved from http://www4.parinc.com/Products/Product.aspx?ProductID=MMSE-2

Paul, S., \& Liu, Y. (2012). Inadequate light levels and their effect on falls and daily activities of community dwelling older adults: A review of the literature. New Zealand Journal of Occupational Therapy, 59, 39-42.

Pegasus Lighting. (2016). A glossary of lighting terms. Retrieved from https://www.pegasuslighting.com/glossary.htm

Perlmutter, M., Bhorade, A., Gordon, M., Hollingsworth, H., Engsberg J., \& Baum, C. (2013). Home lighting assessment for clients with low vision. American Journal of Occupational Therapy, 67, 674-682.

Perlmutter, M. (2012). Home lighting assessment and modifications. Visibility: Education and Research from Envision, 6, 6-9.

Petrofsky, J., \& Cuneo, M. (2008). Correlation between gait, balance, and age when people are standing and walking in normal, subdued, and no light conditions. Physical \& Occupational Therapy in Geriatrics, 26, 23-40.

Pizzi, M., \& Smith, T. (2008). Promoting successful aging through occupation. In M. Scaffa, S. Reitz, \& M. Pizzi (Eds.). Occupation therapy in the promotion of health and wellness (pp. 454-469). Philadelphia, PA: F.A. Davis.

Porter, E. (2007). Scales and tales: Older women's difficulty with daily tasks. Journal of Gerontology: Social Sciences, 62B, S153-S159.

Portney, L., \& Watkins, M. (2009). Foundations of clinical research: Applications to practice $\left(3^{\text {rd }}\right.$ ed.). Upper Saddle River, NJ: Pearson Prentice Hall.

Precision Vision. (n.d.). The Colenbrander Mixed Contrast Card Set. Retrieved from http://www.precision-vision.com/indes.cfm/feature/55 151/colenbrander-acuityintermediate-computer-chart-cat-no-4070---test-instructions-recordingforms.cfm

Precision Vision (2013). Mixed Contrast 40cm (16 in.) Colenbrander Vision Test Card. Retrieved from http://precisionvision.com/index.cfm/product/500_146/mixedcontrast-40cm-16-in-colenbrandervision-test-card.cfm 
Reed, R., Lowrey, C., \& Vallis, L. (2006). Middle-old and old-old retirement dwelling adults respond differently to locomotor challenges in cluttered environments. Gait \& Posture, 23, 486-491.

Rensselaer Polytechnic Institute. (2013). Lighting research center: National lighting product information program. Retrieved from http://www.lrc.rpi.edu/programs/NLPIP/glossary.asp

Rigby, P., \& Letts, L. (2003). Environment and occupational performance: Theoretical considerations. In L. Letts, P. Rigby, \& D. Stewart (Eds.), Using environments to enable occupational performance (pp. 17-32). Thorofare, NJ: SLACK, Inc.

Rizzo, P. (2007). Lighting for universal design. Ultimate Home Design, 7, 51-59.

Robnett, R. (2008). Client factors and their effect on occupational performance in late life. In S. Coppola, S. Elliiott, \& P. Toto (Eds.). Strategies to advance gerontology excellence: Promoting best practice in occupational therapy (pp. 163-197). Bethesda, MD: AOTA Press.

Rogers, W., Meyer, B., Walker, N., \& Fisk, A. (1998). Functional limitations to daily living tasks in the aged: A focus group analysis. Human Factors, 40, 111-125.

Ruya, D. (2015, June 11). Fundamentals of lighting: The function of lighting. Retrieved from http://lumenhub.com/fundamentals-of-lighting/

Sabata, D. (2008). Environmental models used to guide occupational therapy practice. In S. Coppola, S. Elliott, \& P. Toto (Eds.), Strategies to advance gerontology excellence: Promoting best practice in occupational therapy (pp. 263-278). Bethesda, MD: AOTA Press.

Salvi, S., Akhtar, S., \& Currie, Z. (2006). Ageing changes in the eye. Post Graduate Medical Journal, 82, 581-587. Retrieved from http://www.ncbi.nlm.nih.gov/pmc/articles/PMC2585730/

Scheiman, M., Scheiman, M., \& Whittaker, S. (2007). Low vision rehabilitation: A practical guide for occupational therapists. Thorofare, NJ: SLACK, Inc.

Scheiman, M. (2011). Review of basic anatomy, physiology, and development of the visual system. In M. Scheiman (Ed.). Understanding and managing vison deficits: A guide for occupational therapists (pp. 9-16). Thorofare, NJ: SLACK, Inc.

Sekular, R., \& Sekuler, A. (2000). Age-related changes, optical factors, and neural processes. In A. Kazdin (Ed.). Encyclopedia of psychology (Vol. 8, pp. 180183). New York, NY: American Psychological Association/Oxford University Press. Retrieved from http://people.brandeis.edu/ sekuler/papers/APAchapter.pdf 
Simpson, J., \& Tarrant, A. (1983). A study of lighting in the home. Lighting Research and Technology, 15, 1-8.

Skomrock, L., \& Richardson, V. (2010). Simulating age-related changes in color vision to assess the ability of older adults to take medication. The Consultant Pharmacist, 25, 163-170.

Sloan, F., Ostermann, J., Brown, D., \& Lee, P. (2005). Effects of changes in self-reported vision on cognitive, affective, and functional status and living arrangements among the elderly. American Journal of Ophthalmology, 140, 1-12.

Smith, S. (2015). Vision and the coming age. Insight: The Journal of the American Society of Ophthalmic Registered Nurses, 40, 5-10.

Sorensen, S., \& Brunnstrom, G. (1995). Quality of light and quality of life: An intervention study among older people. Lighting Research and Technology, 27, 113-118.

Stark, S., \& Sanford, J. (2005). Environmental enablers and their impact on occupational performance. In C. Christiansen, \& C. Baum (Eds.), Occupational therapy: Performance, participation and well-being (pp. 299-336). Thorofare, NJ: SLACK, Inc.

Stevens, J. (2005). Falls among older adults—Risk factors and prevention strategies. Journal of Safety Research, 36, 409-411.

Stevens, M., Holman, C., \& Bennett, N. (2001). Preventing falls in older people: Impact of an intervention to reduce environmental hazards in the home. Journal of the American Geriatrics Society, 49, 1442-1447.

Strong, S., Rigby, P., Stewart, D., Law, M., Letts, L., \& Cooper, B. (1999). Application of the person-environment-occupation model: A practical tool. Canadian Journal of Occupational Therapy, 66, 122-133.

Talley, V. (2008). Importance of context to occupations of older adults. In S. Coppola, S. Elliott, \& P. Toto (Eds.), Strategies to advance gerontology excellence: Promoting best practice in occupational therapy (pp. 279-318). Bethesda, MD: AOTA Press.

Thoren-Jonsson, A., \& Grimby, G. (2001). Ability and perceived difficulty in daily activites in people with Poliomyelitis sequelae. Journal of Rehabilitation Medicine, 33, 4-11.

Tideiksaar, R. (2003). Best practice approach to fall prevention in community-living elders. Topics in Geriatric Rehabilitation, 19, 199-205. 
Tire Rack. (n.d.). Lighting tech: What is the Kelvin scale and which bulb value should I choose? Retrieved from http://www.tirerack.com/accessories/lighting_tech/techpage.jsp?techid=170

United States Department of Energy. (2012). New lighting standards begin in 2012. Retrieved from http://energy.gov/energysaver/articles/new-lighting-standardsbegin-2012

United States Department of Health and Human Services. (2014). Disability and care needs of older Americans: An analysis of the 2011 national health and aging trends study. Retrieved from https://aspe.hhs.gov/report/disability-and-care-needsolder-americans-analysis-2011-national-health-and-aging-trends-study

Van Bemmel, T., Vandenbroucke, J., Westendorp, R., \& Gussekloo, J. (2005). In an observational study elderly patients had an increased risk of falling due to home hazards. Journal of Clinical Epidemiology, 58, 63-67.

Veitch, J., \& McColl, S. (2001). A critical examination of perceptual and cognitive effects attributed to full-spectrum fluorescent lighting. Ergonomics, 44, 255-279.

Verwey, W., \& Veltman, H. (1996). Detecting short periods of elevated workload: A comparison of nine workload assessment techniques. Journal of Experimental Psychology: Applied, 2, 270-285.

Washington State Department of Ecology. (n.d.). Green home: Healthy home for a healthy environment-lighting. Retrieved from http://www.ecy.wa.gov/programs/swfa/greenbuilding/pdf/Lighting.pdf

Watson, G. (2001). Low vision in the geriatric population: Rehabilitation and management. Journal of the American Geriatrics Society, 49, 317-330.

Wickstrom, G., \& Bendix, T. (2000). The "Hawthorne effect": What did the original Hawthorne studies actually show? Scandinavian Journal of Work Environment and Health, 26, 363-367.

Wilson, M., Smith, N., \& Holmes, P. (2007). The role of effort in influencing the effect of anxiety on performance: Testing the conflicting predictions of processing efficiency theory and the conscious processing hypothesis. British Journal of Psychology, 98, 411-428.

Windham, B., Griswold, M., Fried, L., Rubin, G., Xue, Q., \& Carlson, M. (2005). Impaired vision and the ability to take medications. Journal of the American Geriatrics Society, 53, 1179-1190. 
Woodland, J., \& Hobson, S. (2003). An occupational therapy perspective on falls prevention among community-dwelling older adults. Canadian Journal of Occupational Therapy, 70, 174-182.

Wyman, J., Croghan, C., Nachreiner, N., Gross, C., Stock, H., Talley, K., \& Monigold, M. (2007). Journal of the American Geriatrics Society, 55, 1548-1556.

Yasein, N., Barghouti, F., Irshaid, Y., \& Suleiman, A. (2013). Discrepancies between elderly patients' self-reported and prescribed medications: A social investigation. Scandinavian Journal of Caring Sciences, 27, 131-138.

Yeo, G., \& Neal, A. (2004). A multilevel analysis of effort, practice, and performance: Effects of ability, conscientiousness, and goal orientation. Journal of Applied Psychology, 89, 231-247.

Yeo, G., \& Neal, A. (2008). Subjective cognitive effort: A model of states, traits, and time. Journal of Applied Psychology, 93, 617-631.

Young, D. (2012, September 10). Light the way: Providing effective home modifications for clients with low vision. OT Practice, 17(16), 8-12.

Zijlstra, F. (1993). Efficiency in work behavior: A design approach for modern tools (Doctoral dissertation). Delft University of Technology. Delft, The Netherlands: Delft University Press. 


\section{Appendix A}

\section{PEO Model}

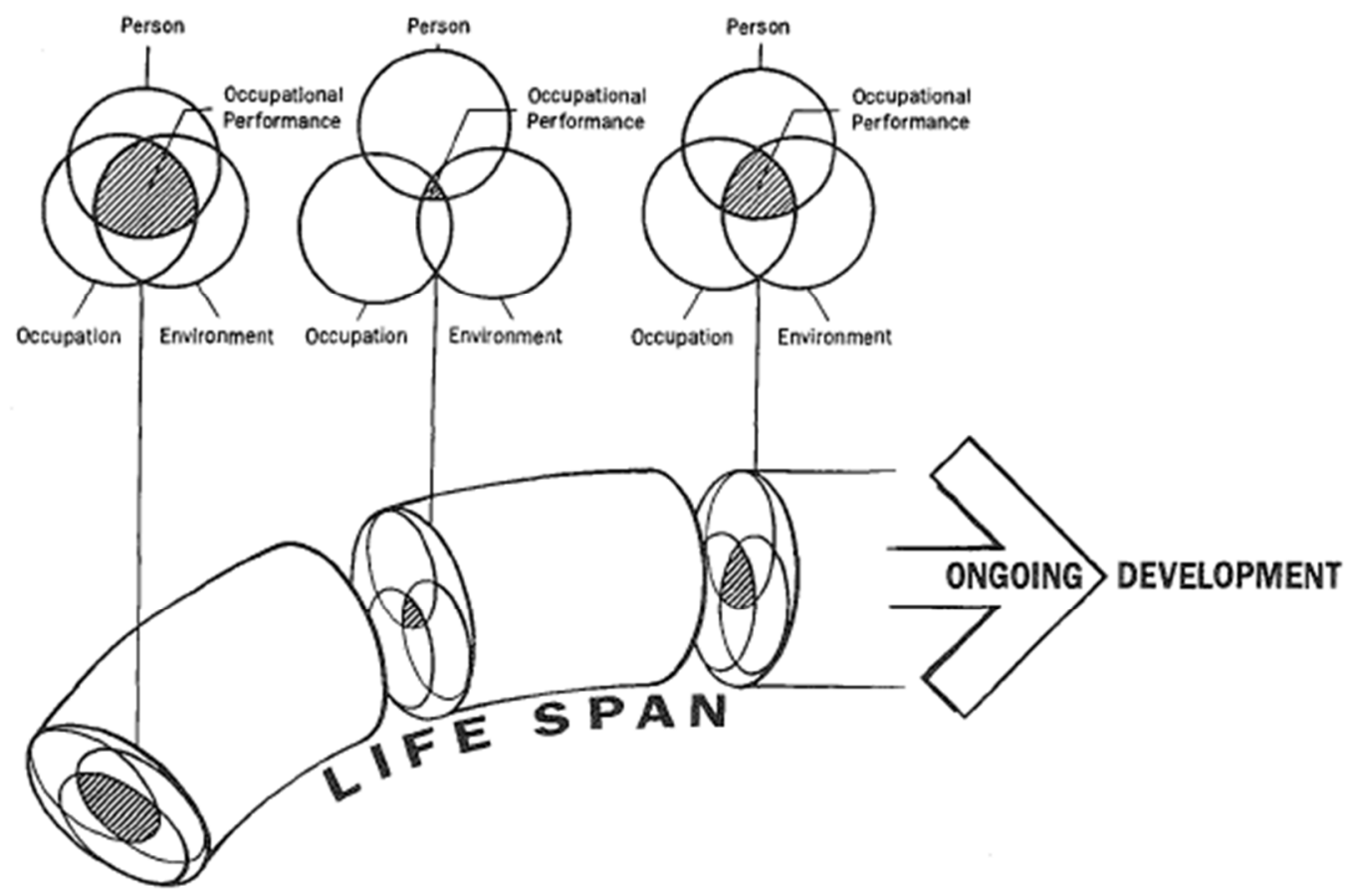

Adapted with permission from "The Person-Environment-Occupation Model: A

Transactive Approach to Occupational Performance," by M. Law et al., 1996, Canadian Journal of Occupational Therapy, 63(1) pp. 9-23, Copyright 1996 by Sage Publishing. 
Appendix B

Flyer for Informational Meeting

\section{Informational Meeting Research Study}

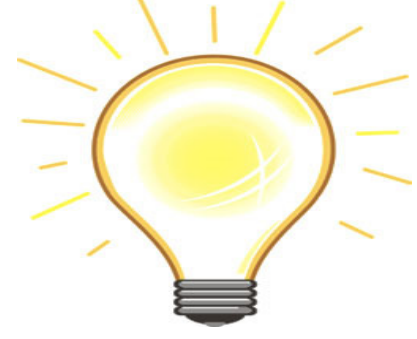

You are invited to attend a meeting to learn how to be part of a research study on how lighting affects your ability to complete common tasks

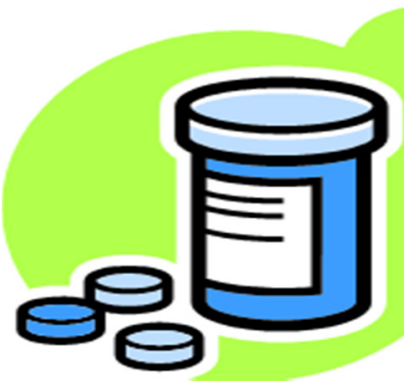

\section{Commons Building}

Date:

\section{Karen James, MS, OTR/L, CAPS}

Occupational Therapist, Certified Aging in Place Specialist and PhD Candidate at Nova Southeastern University, Ft. Lauderdale, FL 
Appendix C

Flyer for Research Study

\section{Invitation to Participate in Research Study}

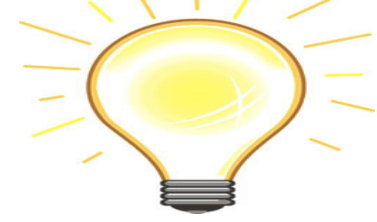

What:

Research Study on Lighting

Why:

Study how lighting affects your ability to perform common tasks

Who: $\quad$ Adults 65 years of age or older

Where: Chaplain's Office in Commons Building

When:

Yes, I would like to participate!

(Please place completed form in Payment Drop Box behind mailboxes):

Name:

Phone \#:

For more information contact:

Karen James, MS, OTR/L

Occupational Therapist, Certified Aging in Place Specialist \& PhD Candidate at Nova Southeastern University, Ft. Lauderdale, FL

(501) 680-4688 
Appendix D

Demographic Information Sheet

Number:

Demographic Information

1. I am a: _ Man _ Woman (Please check one)

2. Age: _ years old

3. Marital status: _ Married _ Widowed _ Single _ Divorced (Please check one)

4. Number of years residing at the Village: years

5. Do you: _ Live alone __ Live with spouse/roommate (Please check one)

6. Highest grade you completed in school:

7. Have you ever been told by a doctor that you have any of the following eye diseases?

(Please check what you have):

_ Cataract _ Macular Degeneration _ Diabetic

Retinopathy

_ Glaucoma _ Other___ (please

list)

_ Do not have eye disease 
8. I use a: _ Walker _ Cane _ Wheelchair (Please check all that apply)

Do not use walker, cane or wheelchair

9. Have you fallen in the past year? _ Yes _ No (Please check one)

10. If you have fallen in the past year, please indicate what you believe caused you to fall: (Please check all that apply)

\begin{tabular}{|l|l|}
\hline & Did not see an object in my path \\
\hline & Tripped or ran into object \\
\hline Lighting condition such as dim lighting or glare \\
\hline Dizziness, loss of balance or other medical condition \\
\hline Carelessness/not paying attention \\
\hline Other (list): \\
\hline
\end{tabular}

11. Please check all the activities below you have difficulty with:

\begin{tabular}{|l|l|}
\hline & Reading a book/magazine \\
\hline Locating a listing in a telephone book \\
\hline Paying bills \\
\hline Writing checks \\
\hline Preparing food \\
\hline Grooming (applying make-up or shaving) \\
\hline Participating in leisure activities such as bingo, knitting, etc. \\
\hline
\end{tabular}




\begin{tabular}{|l|l|}
\hline & Doing laundry \\
\hline Taking medication \\
\hline Other: \\
\hline
\end{tabular}

The researcher will complete the following:

MMSE-2:

Colenbrander Mixed Contrast Card:

Corrective lenses: Yes (_ glasses __ contact lenses) No 
Appendix E

Study Record

Study Record

$\begin{array}{llll}\text { Light Condition (circle one): } & \text { A } & \text { B } & \text { C }\end{array}$

Trial \#1: $\quad$ fc

\begin{tabular}{|l|l|l|}
\hline Task & Time & RSME \\
\hline Rx Label & & \\
\hline Pills & & \\
\hline Socks & & \\
\hline TOTAL & & \\
\hline
\end{tabular}

Trial \#2: $\quad$ fc

\begin{tabular}{|l|l|l|}
\hline Task & Time & RSME \\
\hline Rx Label & & \\
\hline Pills & & \\
\hline Socks & & \\
\hline TOTAL & & \\
\hline
\end{tabular}

Trial \#3: $\quad$ fc

\begin{tabular}{|l|l|l|}
\hline Task & Time & RSME \\
\hline Rx Label & & \\
\hline Pills & & \\
\hline Socks & & \\
\hline TOTAL & & \\
\hline
\end{tabular}


Appendix F

Cards for the Order of the Lighting Conditions

\section{Cards Indicating Order of Lighting Conditions}

\section{Card A}

100 footcandles

125 footcandles

400 footcandles

\section{Card B}

400 footcandles

125 footcandles

100 footcandles

\section{Card C}

125 footcandles

400 footcandles

100 footcandles 
Appendix G

Contents of Testing Sets

\section{Trial Set}

\section{Contents of Testing Sets}

Socks

Gold Toe Cotton Dress Socks

Pills

Nature's Blend Niacin $250 \mathrm{mg}$

Walgreens Aspirin 325 mg

Rising Pharmacy Sodium Bicarbonate $650 \mathrm{mg}$

Assured All Day Allergy Relief Cetirizine Hydrochloride 10 mg

Prescription label

Allan Jefferson

Take 1 capsule by mouth once a week

Vit D 50, 000 IU D2 (Ergo) caps

\section{$\underline{\text { Set } 1}$}

Socks

Silver Toe-A Gold Toe Brand Cotton Wide Rib

Pills

Rugby Magnesium Oxide 400 mg

Quality Plus Aspirin $325 \mathrm{mg}$

Certified Brand Gas Relief Simethicone $80 \mathrm{mg}$

Kroger Maximum Strength Heartburn Prevention Famotidine 20 mg Prescription label

Janet Miller

Take 1 tablet by mouth every day

Diltiazem $90 \mathrm{mg}$ tablets 


\section{Set 2}

Socks

\section{Stafford Cotton Rich}

Pills

Assured Expectorant Mucus Relief Guaifenesin 400 mg

Mason Distributing Potassium Gluconate $595 \mathrm{mg}$

Extra Strength Rolaid Antacid Calcium Carbonate USP 675 mg \& Magnesium Hydroxide USP $135 \mathrm{mg}$

Equate Famotidine Acid Reducer $20 \mathrm{mg}$

Prescription label

Peter Franks

Take 1 capsule by mouth three times daily with food

Cephalexin $250 \mathrm{mg}$ capsules

\section{$\underline{\text { Set } 3}$}

Socks

Gold Toe Fluffies

Pills

Consolidated MI Sodium Chloride $1 \mathrm{gm}$

Equate Acetaminophen Regular Strength $325 \mathrm{mg}$

DRx Choice Gas Relief Anti-Gas Simethicone 80 mg

Dramamine Original Formula $50 \mathrm{mg}$

Prescription label

Marie Collins

Take 2 capsules by mouth every night at bedtime

Celebrex 200 mg capsules 